\title{
Local Protein Turnover As a Regulatory Mechanism of Growth and Collapse of Neuronal Growth Cones
}

\author{
Dissertation \\ Zur Erlangung des Doktorgrades \\ der Mathematisch-Naturwissenschaftlichen Fakultäten \\ der Georg-August-Universität Zu Göttingen
}

vorgelegt von

\section{Sundar Ganesan}

aus Kancheepuram (Indien)

Göttingen 2005 
D7

Referent:

Prof. Friedrich-Wilhelm Schürmann

Koreferent:

Prof. Detlef Doenecke

Tag der mündlichen prüfung: 


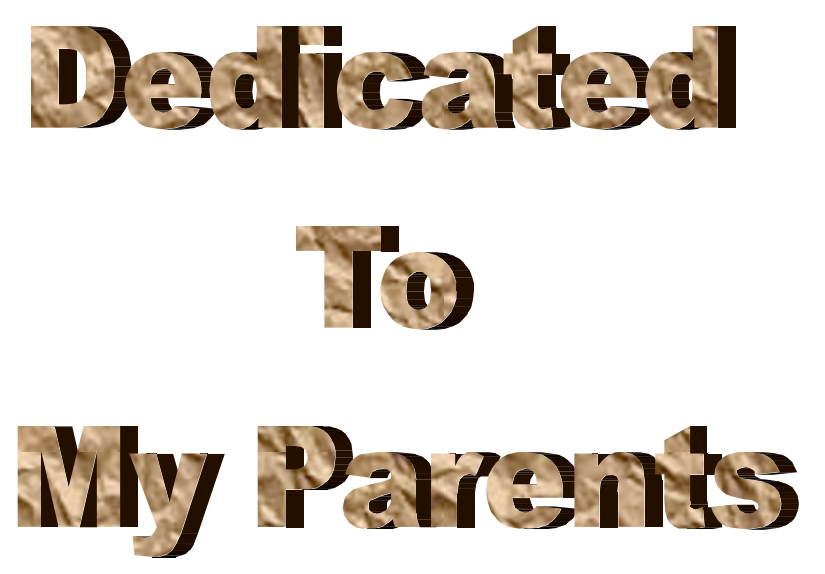


Aim of the study..............................................................................................................004

Hypothetical model for protein turnover- Thesis Overview.......................006

Chapter 1: INTRODUCTION.

1.1. Nervous System and Axonal Path-finding..........................007

1.1.1. Neurons and their components....................................................007

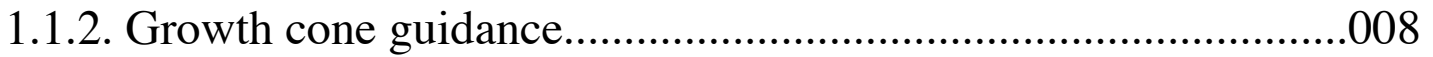

1.1.3. Signal transduction at the growth cones......................................010

1.1.3.1. Rho GTPases and Growth Cone Actin Dynamics

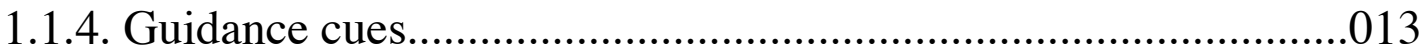

1.1.4.1. Repulsive guidance cues

1.1.4.1.1. Lyso Phosphatidic Acid, 1-oleoyl-2-hydroxy-sn-

glycero-3-phosphate (LPA)

1.1.4.1.2. Semaphorins and Neuropilins

1.1.4.1.3. Neuropilins

1.1.4.2. Attractive Guidance Cues

1.1.4.2.1. Netrins and Their Receptors

1.2. Local protein turnover mechanisms in growth cone guidance.......019

1.2.1. Axonal Transport mechanism in Growth cone guidance. 


\subsubsection{Slow Vs Fast axonal transport with examples}

1.2.1.1.1. Neurofilament-M subunit

1.2.1.1.2. Alpha Synuclein

1.2.2. Protein synthetic machinery in the growth cone guidance

1.2.3. Protein degradation machinery in the growth cone guidance (Ubiquitin Proteasome System (UPS))

\subsubsection{Ubiquitin}

1.2.3.2. Ubiquitination in the nervous system

1.2.3.3. Proteasome mediated protein degradation in neuronal axons

1.2.4. Protein folding machinery in the growth cone guidance

1.2.4.1. Molecular chaperones OR Heat Shock Proteins (HSPs)

1.2.4.2. BAG-1 (Bcl-2-associated athanogene)

\subsection{Local protein turnover as regulatory mechanism for} growth cone guidance.

1.4. Imaging techniques used:FRET and FLIM.................................036

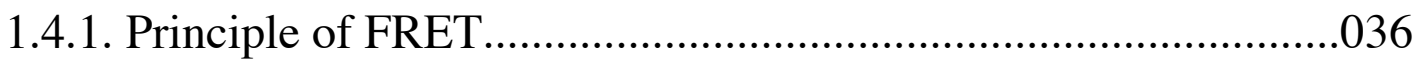

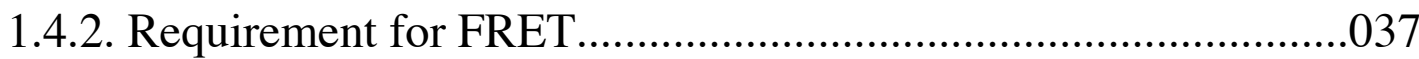

1.4.3. FRET imaging techniques and biological applications...............037

1.4.4. FLIM (Fluorescent Lifetime Imaging Microscopy).....................039

1.4.4.1. Principle of FLIM

1.4.4.2. Frequency Domain FLIM

1.4.4.3. Time-domain FLIM 
2.1. Chemicals............................................................................................................041

2.2. Cell culture medium, inhibitors and chemicals.................................044

2.3. Molecular biology chemicals, competent cells and vectors...............045

2.4. Enzymes..............................................................................................................046

2.5. Antibodies.........................................................................................................048

Chapter 3: METHODS...........................................................................050

3.1. Molecular biology protocol....................................................................050

1.1. Buffers and solutions

1.2. Restriction digestion Buffers

1.2. Gel loading buffer

1.4. PCR buffers

1.5. Cloning buffers.

1.6. Chemical competent cells preparation

1.7. Preparation of electro-competent cells

1.8. Determining the concentration of DNA.

1.9. Transformation of DNA to chemical competent cells.

1.10. Transformation of DNA to Electro competent cells

1.11. Preparation of Glycerol stock

1.12. Agarose gel 
1.13. Mini preparation of plasmid DNA

1.14. Restriction Digestion DNA.

1.15. Polymerase chain reaction (PCR)

1.16. Random Mutagenesis of EYFP

1.17. Site directed mutagenesis

1.18. Dpn1 digestion

1.19. Molecular Cloning and Vector construction

1.20. Ligation

3.2. Biochemical methods.

2.1. Buffers and solutions

2.2. Protein expression and purification

2.3. Protein quantification using BIORAD reagent

2.4. Spectro-photometry

2.5. SDS-PAGE (Poly Acrylamide Gel Electrophoresis)

2.6. Spectro-fluorimetry

\subsection{Cell culture methods}

3.1. Buffers and solutions

3.2. Coating and seeding neurons for collapse assay

3.3. Fixing cell's with PFA or METHANOL

3.4. Transfection

3.5. PC12 cells differentiation and transfection

3.6. Cryo-preservation of cells 
3.4. Western blotting. .069

4.1. Preparation of cell lysate

4.2. Blot transfer

3.5. Imaging Techniques .071

5.1. Confocal and flourescence microscopy

5.2. Immuno cytochemistry or Immuno fluorescence

5.3. FqRET analysis

5.4. Fluorescence Lifetime Microscopy: TD-FLIM

Hypothetical model of local protein turnover- Thesis Overview. .074

Chapter 4: RESULTS. .075

\subsection{Generation of biosensors for the study of local protein....................075 turnover in the growth cone}

4.1.1. Biosensor to analyse protein transport.......................................075

4.1.1.1. FRET by FLAP Principle...................................076

4.1.2. Biosensor to analyse protein ubiquitin machinery.....................078

4.1.2.1. Generation of REACh by mutating the Yellow Fluorescent.........078 Protein (YFP)

4.1.2.2. Spectrofluorimetric and Lifetime analysis Of Mutant...... .079 Protein

4.1.2.3. Lifetime analysis on REACh protein in solution at different .081 wavelengths

4.1.2.4. A REACh-based single-cell FRET sensor for protein .086 ubiquitination 
4.1.2.5. Biochemical and immuno-cytochemical characterisation of........086 REACh protein ubiquitination

4.1.2.6. Lifetime analysis of REACh-FRET sensors in multiple .088 spectral windows

4.1.2.7. Donor Photobleaching kinetics as a indicator for the .092 occurence of FRET

4.1.2.8. FqRET Fluorescence quenching Reasonance Energy Transfer....094

4.1.3. Biosensor to analyse protein folding and chaperone activity....096

4.1.3.1. Creation of folding mutant and Recombinant protein. .096 characterisation

4.1.3.2. Response of Mammalian cells for Foldase sensor under .099 Stress related conditions

4.1.3.3. Structural characterisation of folding mutant 100

4.1.3.4. Measuring the folding efficiency of folding mutant in 101 mammalian cells

4.1.3.5. Chaperone-mediated folding assay of foldase sensor in 101 mammalian cells

4.1.3.6. Foldase sensor in sensing the activity HSP70 using BAG1 a 103 Co-chaperone expressing stable cell lines

\subsection{Analysis of growth cone protein synthesis, transport,} degradation and folding in neurons and neuron-like PC12 cells.....107

4.2.1. Analysis of protein transport machinery in neuronal growth cone. 108

4.2.1.1. Synaptophysin p38 tagged FLAP Sensor in transport assay

4.2.2. Analysis of protein degradation machinery in the growth cone.

4.2.2.2. Ubiquitination of growth cone like structures in PC12 cells 110

4.2.2.3. Growth cone of PC12 cells show decrease in protein content. 
4.2.2.4. Growth cones of Cortical Neurons show chemotropic response. 117 to protease and proteasome Inhibitors

\subsection{Collapse assay}

4.2.3. Analysis of protein synthetic machinery in the growth cone.....123

4.2.3.1. Growth cone like structures of PC12 cells has protein synthetic....123 machinery

4.2.4. Analysis of protein folding in cells and in the growth cone........124

4.2.4.1. Folding Mutant can induce Heat Shock Protein 70 in Bag1........124 Transfected CSM cells

4.2.4.2. HSP70 overexpression has no effect on local protein folding at......126 the PC12 cells growth cone

4.2.5. Functional analysis of HSP70 in aggregation induced...............128 neuropathology

4.2.5.1. HSP 70 overexpression prevents aggregation-induced cell. Death

4.2.5.2. Protein aggregation and cyto-toxicity are induced by..... 130 phosporylation

\section{Chapter 5: DISCUSSION}

\subsection{The use of biosensors for the study of local events of} protein transport, ubiquitination, folding and synthesis.

5.1.1. Development of axonal transport marker to study the contribution of cell soma for local protein pool in the growth cones.

5.1.2. REACh based FRET sensors for protein ubiquitination.

5.1.2.1. REACh Based Ubiquitination assay in biological systems 
5.1.2.3. Advantages of REACh acceptors for FRET based measurements

5.1.3. Biosensor to detect the Chaperone assisted local protein Folding 140

5.1.3.1. Protein folding dependent foldase sensor. 140

5.1.3.2. Foldase sensor in sensing BAG1 induced HSP70 induction. 140

5.1.3.3. Foldase sensor in sensing the differences of inducibility of. 141 chaperone

5.2. Observation on protein turnover in the growth cone. 142

5.2.1. Protein translation and degradation occurs locally in the growth cone

5.2.2. Chemotropic response of neuronal growth cone mediated by rapid local protein turnover.

5.2.3.. Chaperone induction is required for neuronal survival. 146

5.3. Conclusions and implications of local protein turnover as a regulatory mechanism for growth cone function. 148

Hypothetical Model of local protein turnover- Thesis Overview..... 151

SUMMARY and CONCLUSION. 152

BIBLIOGRAPHY. 154

BIO-DATA 


\section{Results pictures and tables}

1. FLAP tag based FRET assay on axonal transport...............................................077

2. Spectrofluorimetric characterisation of REACh mutants...................................080

3. Lifetime analysis of REACh mutants in protein solution....................................082

4. Lifetime analysis of REACh mutants in mammalian cells.................................084

5. Antibody colocalisation of REACh ubiquitination of GFP-PEST.......................087

6. FRET measurement of REACh ubiquitination using filter free system.................090

7. FRET measurement of REACh ubiquitination using GFP band pass filter..........091

8. Donor photobleaching analysis of REACh ubiquitination.

9. Characterisation of cDYFP folding efficiencies in bacterial and mammalian cells.

10. Folding response of cDYFP in mammalian cells is dependent on HSP70. 102

11. Foldase sensor characterisation in BAG1 expressing stable cell line. 105

12. FLAP tag based FRET assay of axonal transport-synaptophysin p38 tag. 109

13. Protein ubiquitination assay in Neuron like PC12 cells

14. FRAP analysis on growth cone protein synthesis- Control cells untreated. 114

15. FRAP analysis on growth cone protein synthesis- Nocodazole treated cells.......115

16. FRAP analysis on growth cone protein synthesis- Taxol treated cells 116

17. Effect of protease and proteasome inhibitors on LPA induced collapse.

18. LPA induced collapse and the effect of inhibitors on growth cone collapse.

19. Effect of protease and proteasome inhibitors on Sema3a induced collapse.

20. Effect of inhibitors on Netrin1 induced growth or repulsion.

21. Response of folding mutant induced HSP70 expression in BAG1. 125 expressing cells

22. Local response of HSP70 mediated folding at the growth cone of PC12 cells 
23. Effect of Protein aggregation in HSP70 induction and cell survival.

129

\section{Discussion pictures}

24. Advantages of REACh mutants in FRET based studies....................................134

25. REACh based single cell protein ubiquitination assay.......................................136

26. Intensity based FRET using REACh acceptor..................................................138

\section{Tables}

Table 1: Lifetime analysis with protein solution at multiple wavelength........................085

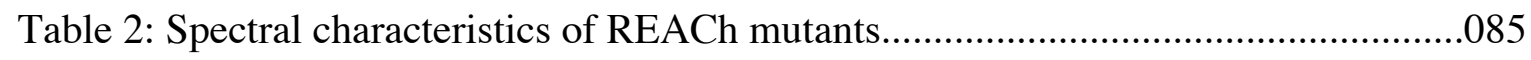




\section{Abbreviations}
AA
Amino acid
Amp
Ampicillin
APS
Ammonium per sulphate
ATP
Adenosine 5' triphosphate
BSA Bovine serum albumin
Bcl2 B-cell leukemia factor
BAG Bcl2 associated athanogene 1
cAMP Adenosine 3'-5' - cyclin mono phsophate
DNA Deoxy ribonucleic acid
cDNA complimentary DNA
CHO Chinese Hamster Ovary
$\mathrm{Ct} \quad$ Control
ddH2O Double distilled water
DMSO Dimethy sulfoxide
dNTPs Deoxy nucleotide triphosphate
DTT Dithiothreitol
E. coli Escherichia coli
EDTA Ethylene diamine tetra acetate
EGF Epidermal growth factor
EGTA Ethyleneglycol-bis- ( $\beta$-Aminoethylether)- N,N,N',N'- Tetraacetate
EtOH Ethanol
FLIM Fluorescence Lifetime Imaging
FRET Fluorescence Reasonance Energy Transfer
FRAP Fluorescence Recovery After Photobleaching 


\begin{tabular}{|c|c|}
\hline GAP & Guanosine 5'-triphosphate activating protein \\
\hline GEF & Guanosine nucleotide exchange factor \\
\hline GDP & Guanosine 5'-diphosphate \\
\hline GST & Glutathione-S-Transferase \\
\hline GTP & Guanosine 5'-triphosphate \\
\hline HEPES & N-2-Hydroxyethylpiperazin-N'-2-ethanesulfonic acid \\
\hline HeLa & Henrietta Lacks \\
\hline HRP & Horse Radish Peroxidase \\
\hline $\operatorname{IgG}$ & Immunoglobulin $\mathrm{G}$ \\
\hline IPTG & Isopropyl- $\alpha$-D-Thiogalactopyranoside \\
\hline $\mathrm{Kb}$ & Kilobase \\
\hline Kda & Kilo Dalton \\
\hline $\mathrm{L}$ & Liter \\
\hline LB & Luria Bertani \\
\hline M & Molar \\
\hline MCF-7 & Mammary Cell Carcinoma Fibroblast-7 \\
\hline $\mathrm{mg}$ & Milligram \\
\hline $\min$ & Minute \\
\hline $\mathrm{ml}$ & Milli liter \\
\hline $\mathrm{mM}$ & Milli Molar \\
\hline $\mathrm{nm}$ & Nano Meter \\
\hline NGF & Nerve Growth Factor \\
\hline PAGE & Poly Acrylamide Gel Electrophoresis \\
\hline PCR & Polymerase Chain Reaction \\
\hline $\mathrm{pH}$ & Negative logarithm of $\mathrm{H}+$ concentration \\
\hline
\end{tabular}


PKA Protein Kinase A

PIP Phospho Inositol Phosphate

PMSF Phenyl methyl sulphonyl fluoride

RT Room Temperature

SDS Sodium Dodecyl Sulphate

Sec Seconds

TAE Tri-Acetate-EDTA Buffer

TCA Tri Chloro Aceticacid

TE Tris-EDTA-Buffer

TEMED N,N,N',N'-Tetra Methyl ethylenediamine

Tris Tris-(hydroxymethyl)-aminomethane

U Unit

UV Ultra Violet

v/v $\quad$ Volume/Volume

w/v Weight/Volume

REACh Reasonance Energy Accepting Chromoprotein

Wt wild type

$\mu \mathrm{M} \quad$ Micro Molar

$\mu \mathrm{g} \quad$ Micro Gram

FRAP Fluorescence Recovery After Photobleaching

GFP Green Fluorescent Protein 


\section{Aim of the study}

\section{Main project:}

Elucidating the local protein turnover as the regulatory mechanism of growth and collapse of neuronal growth cone.

The aim of this project is to characterise the local protein turnover mechanism and their regulatory role in growth cone navigation.

In this project we would like to focus on:

1. The mechanism of growth cone collapse towards various guidance cues in cortical neuron, as a model system.

2. To find out the role of protein turnover machinery in mediating the effect of growth cone guidance, combined with inhibitor studies

3. Using various microscopic techniques to study and explain the role of local protein turnover mechanism and to study the interaction of growth cone proteins.

\section{Project1: Construction of FRET-based axonal transport marker}

In this project we like to create a biosensor that can explain the axonal protein transport and the role of axonal transport in mediating the local response of growth cone guidance. We tried to exploit the FRET based microscopic technique to study the movement of molecules within short range of axons and a specific biosensor design to study the axonal transport. 


\section{Project2: Generation of FRET based biosensor protein ubiquitination machinery}

In this we project we attempt to create novel non-fluorescent variant of green fluorescent protein to design a FRET based biosensor to study the active ubiquitination machinery in living cells. Further using this sensor we like to study the fuctional role of protein ubiquitination, in chemotropic guidance of axonal growth cone. Since FRET based study reveal the protein to protein interaction in a micro volume, the protein ubiquitination sensor will be a novel tool to study the local mechanism of degradation in the growth cone guidance.

\section{Project3: Creation of folding dependent sensor to detect chaperone activity}

To create a intensity based protein folding sensor to study the functional role of local chaperone activity in growth cone guidance. We will use the standard molecular biology and biochemistry to create and characterise a fluorescent sensor, that can sense the inducibility of molecular chaperone in cell systems. Further we would like to extend the use of this biosensor in the study of stress response and neuronal survival under neurodegenerative disease model systems namely, Alzhimer's and Parkinsons's diseases. 


\section{Hypothetical model for local protein turnover: Project Overview}

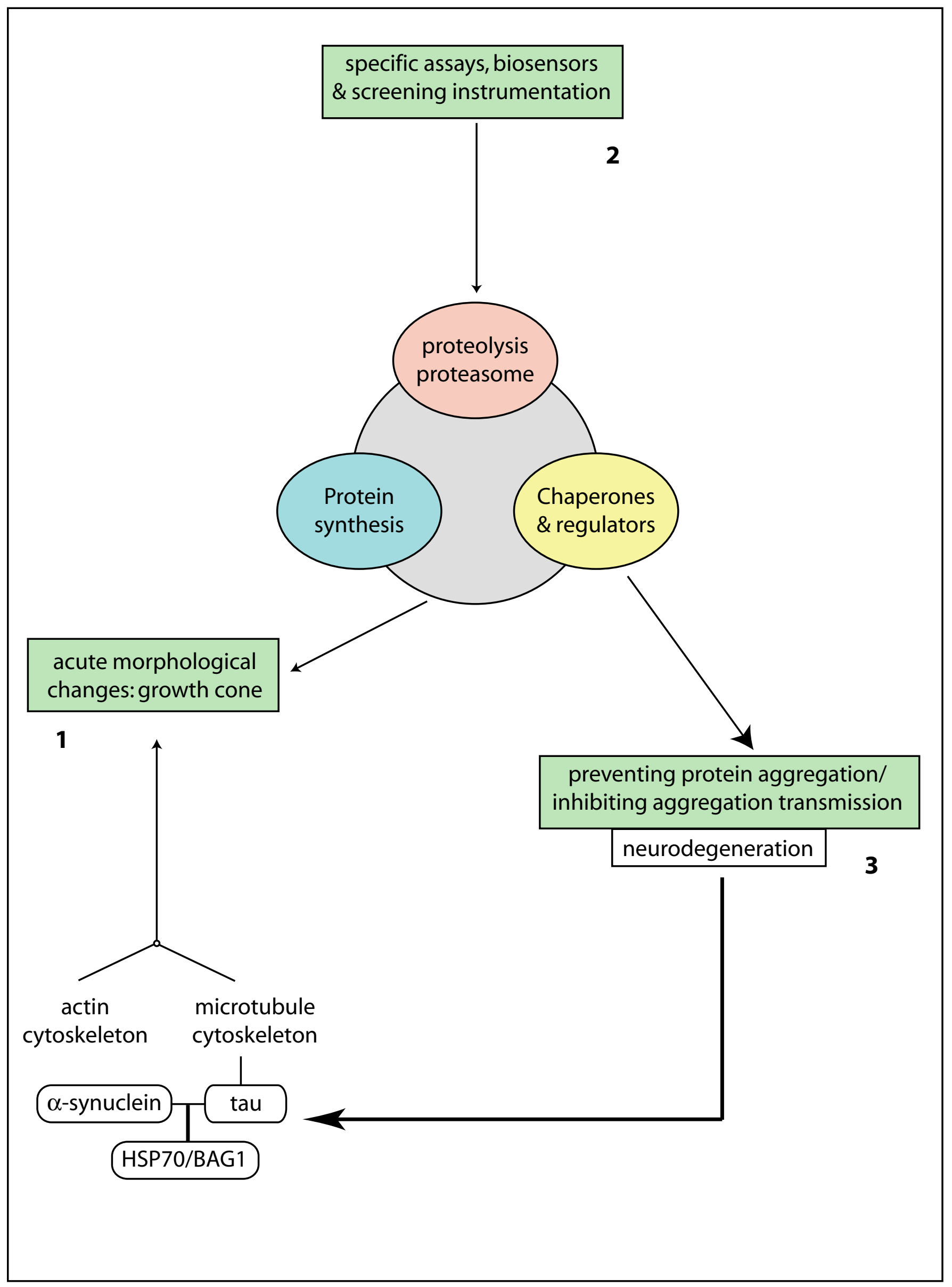




\section{Chapter 1: INTRODUCTION}

\subsection{Nervous System and Axonal Path-finding}

\subsubsection{Neurons and their components}

Neurons are the fundamental building blocks of the nervous system that provides the link between different regions of brain and parts of the body to coordinate their function in an orchestrated fashion. The anatomical and physiological system of the body becomes functional only when this precision in wiring of the nervous system is achieved.

The unique feature of the nervous system is the complex morphological phenotype of neurons and the intricate network of specific neuronal connections. Each neuron possesses multiple compartments like dendrite, axon, cell soma and growth cones each with its own physiological significance for brain function. To be functional, neurons have to interact and find their target connections to interrelate their physiological input. The complex network of neuronal connections of compartments involving dendrites and axons is formed through their motile finger like structures called growth cones.

For decades, the field of neuronal navigation has gained momentum and led to an understanding of how neurons are targeted to the final connection under the influence of guidance molecules. Varied responses of navigating growth cones to different guidance molecules are necessary to find their appropriate target and strengthening of synaptic connections by the activity-dependent status of growth cone once it finds its appropriate 
target. What is puzzling still is the identity of the internal complex network of signaling that alters its functional behavior in a spatio-temporal manner within the neuron and in the growth cone upon its myriads of encounters with guidance cues.

\subsubsection{Growth cone guidance}

Axonal path finding during neuronal development is mediated by the growth cone, which integrates and responds to guidance cues in the extra-cellular environment. The growth cone is a specialized region with motile finger like projections at the tip of the axons that are guided by molecular cues (both positive (attractive) and negative (repulsive) cues) by selectively stabilizing and destabilizing the actin cytoskeleton in filopodia and lamillipodia to achieve directional growth (Bentley and O'Connor, 1994; Lin et al., 1994). With formation of appropriate connections between and their target cells is a fundamental step during development of invertebrate and vertebrate nervous systems.

Figure 1. Guidance mechanism and Growth cone components

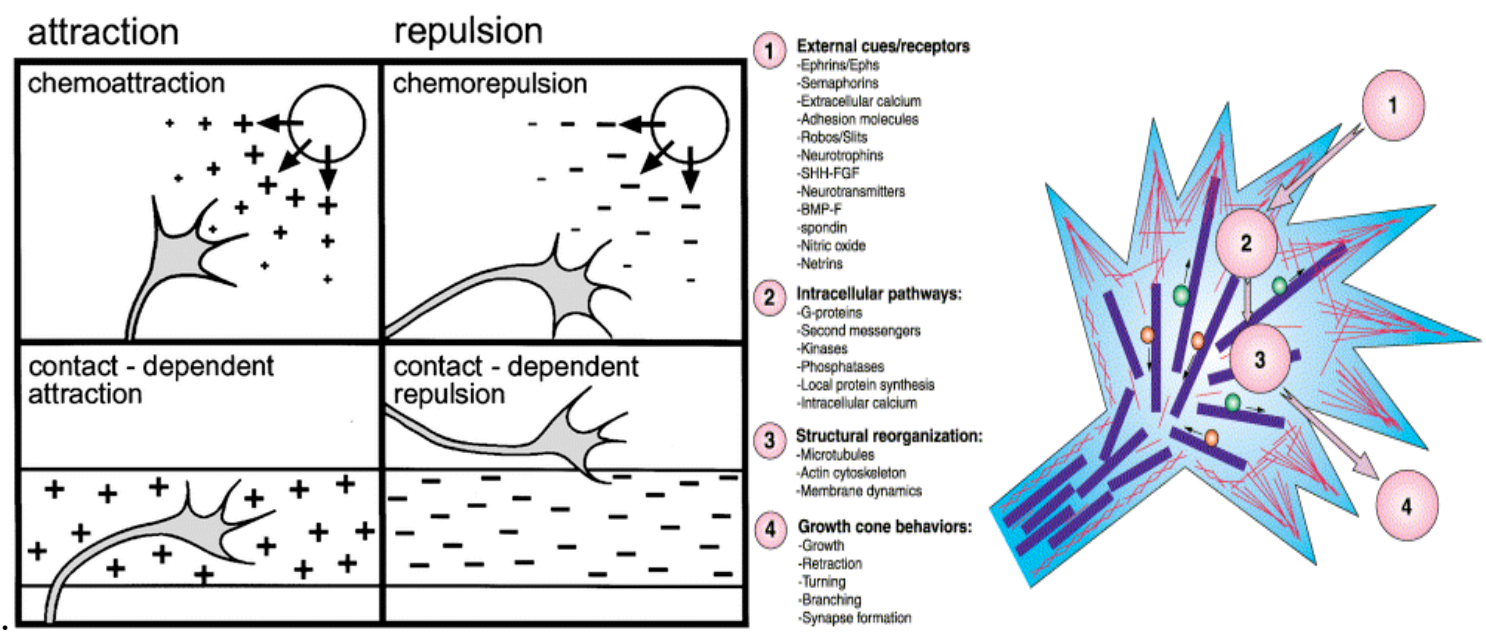

(Mueller BK et al Annul Rev Neuroscience, 1999) 
Normal functioning of the nervous system requires precision in wiring together the structures often separated by large distance ranges from millimeters to meters. Two types of mechanisms are necessary to achieve this precision: early acting mechanisms independent of neuronal activity and late acting, activity -based, refinement mechanisms (Goodman \& Shatz 1993, Tessier-Lavigne \& Goodman 1996). Growth cones are guided to their target by the classical mechanisms like chemo-repulsion, chemo-attraction, contact dependent repulsion and contact dependent attraction

To set up the projection pattern in the nervous system, axonal growth cones must find their targets by traveling long and complex pathways. An important principle to help in this complex path-finding process is the presence of intermediate targets, which break the journey into smaller segments (Tessier-Lavigne and Goodman, 1996). Axons are initially guided toward such intermediate targets, then grow past them and adopt a new trajectory on the other side. For this, growth cones are believed to change their response to multiple guidance cues as they pass from one side of an intermediate target to the other. Comparable changes in responsiveness to multiple cues must also occur as projecting axons reach their final target. The growth cone, a finger like sensory structure at the tip of the outgrowing axon, is the central player mediating these axon guidance decisions. The growth cone senses guidance cues in its environment and translates this information into changes in the cytoskeleton that determine the direction of outgrowth. Upon reaching the appropriate target, the growth cone differentiates into a presynaptic terminal as synapse formation begins. From then synaptic connections are themselves dynamically regulated by activity and competition with other synapses (Sanes and Lichtman 1999, Zhang and Poo 2001). 


\subsubsection{Signal transduction at the growth cones}

The growth cone as a neuronal compartment has important functions that are independent of its cell soma. The growth cone possesses intricate mechanism of steering and altered functional behavior depends on its signaling intermediate. The guidance of axons during the establishment of the nervous system is mediated by a variety of extracellular cues that govern cytoskeletal dynamics in axonal growth cones.
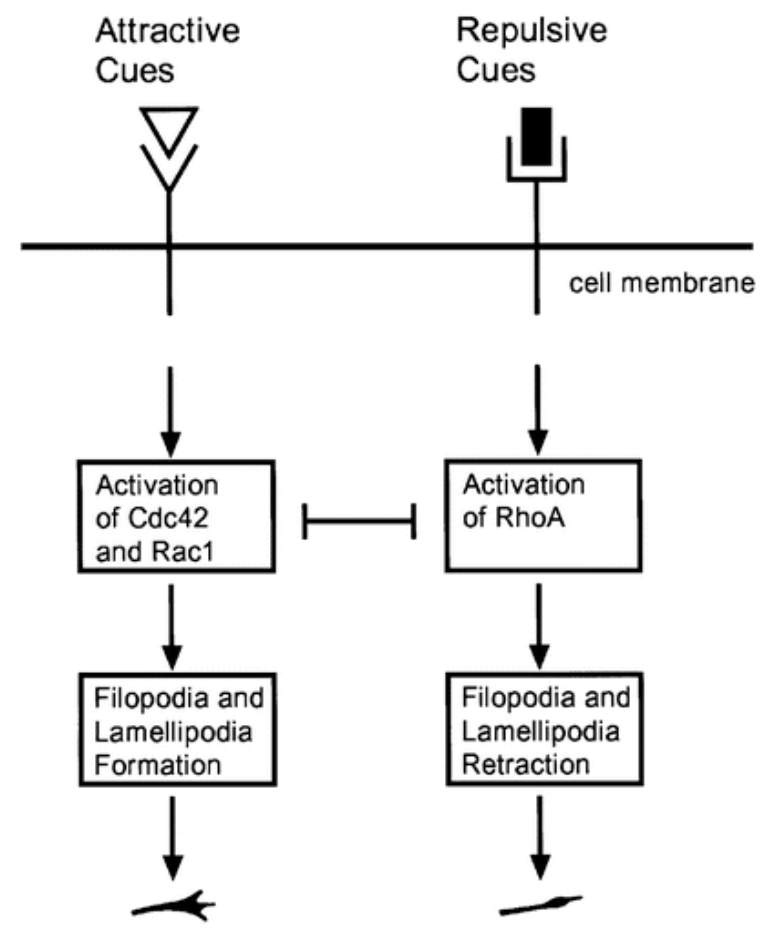

(Mueller BK et al Annu Rev Neuroscience, 1999)

A large number of these guidance cues and their cell-surface receptors have now been identified, and the intracellular signaling pathways by which these cues induce cytoskeletal rearrangements are well defined. At the leading edge of a growing axon is the growth cone, a structure capable of sensing and rapidly responding to its environment. The growth cone is composed of lamellipodia, which contain cross-linked networks of actin filaments, and 
filopodia, tensile structures consisting of bundled F-actin that sense the extracellular environment. This peripheral actin network is associated in the proximal portion of the growth cone with microtubules located in the distal region of the axon shaft and contributes to the assembly and translocation of microtubules into more proximal regions of the growth cone (Mallavarapu \& Mitchison 1999, Mitchison \& Kirschner 1988, Suter \& Forscher 2000). The growth cone advancement and retraction is controlled by the regulation of actin polymerization at the leading edge of filopodia and lamellipodia, of actin depolymerization in proximal regions of the growth cone and of the rate of F-actin retrograde flow within both filopodia and lamellipodia. It is amazingly, within the growth cone, attractive guidance cues can promote actin polymerization and prevent retrograde actin flow, while in contrast repulsive guidance cues can have reciprocal effects on these processes.

\subsubsection{Rho GTPases and Growth Cone Actin Dynamics}

There is ample evidence that axon guidance cue signaling involves the action of proteins belonging to the Rho family of small GTP-binding proteins, key regulators of actin cytoskeletal dynamics. The Rho family GTPases orchestrate actin filament assembly and disassembly through the control of actin polymerization, branching, and depolymerization. Moreover, Rho family members direct actin-myosin-dependent contractility, controlling the retrograde flow of F-actin within the growth cone that control cytoskeletal rearrangements (reviewed in Dickson 2001, Hall 1998, Luo 2000, Luo 2002). The best studied Rho GTPases, Cdc42, Rac, and RhoA, have been implicated in the control of lamellipodial and filopodial dynamics in fibroblasts and, more recently, in neuronal growth cones. Like all small GTP-binding proteins, Rho GTPases cycle between active and inactive states through 
the binding of guanine nucleotides. In their GTP-bound states, Rho GTPases recruit effector proteins to the membrane and are thus considered active. Hydrolysis of GTP to GDP by their intrinsic GTPase activity disrupts GTPase binding to effectors and thereby inactivates these GTPases

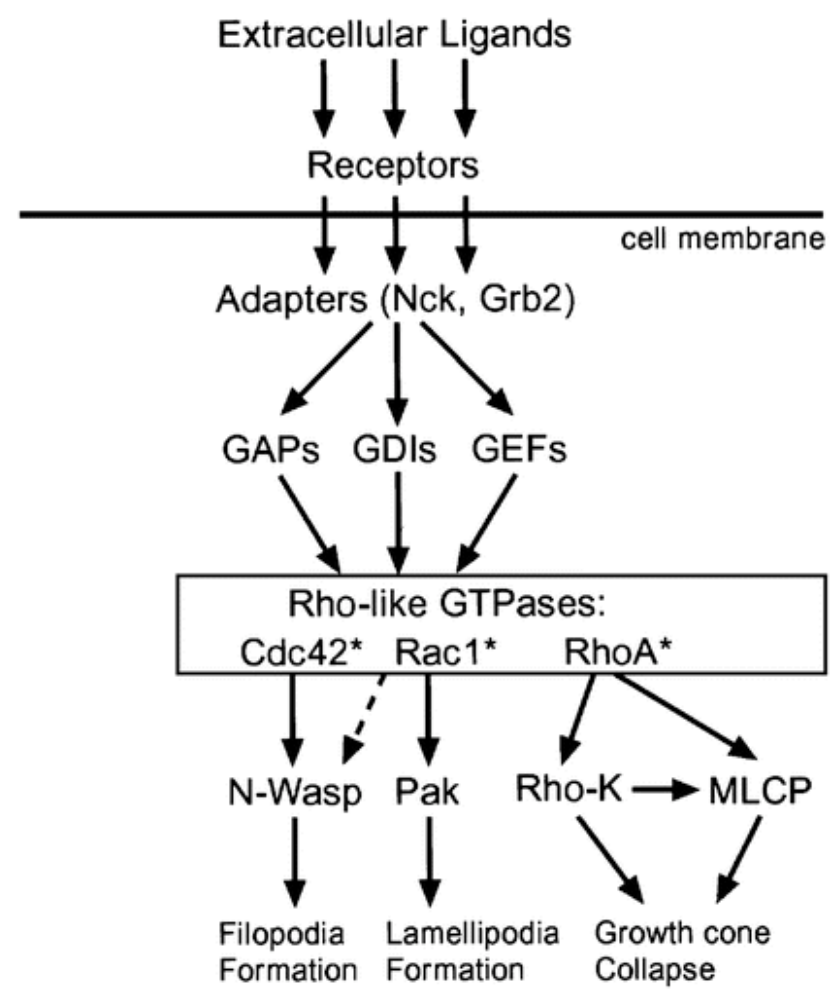

(Mueller BK et al Annu Rev Neuroscience, 1999)

The activity of Rho GTPases is controlled by the opposing actions of guanine nucleotide exchange factors (GEFs) and GTPase activating proteins (GAPs). GEFs facilitate the exchange of GDP to GTP, thereby turning on Rho GTPase signaling, whereas GAPs turn off Rho GTPase signaling by activating their endogenous GTPase activities. Axon guidance receptors can be directly or indirectly coupled to GEFs and GAPs, affording these receptors direct control over Rho GTPases. The large number of GEFs and GAPs, many of which are expressed uniquely both temporally and spatially in the nervous system, contrasts 
with the relatively small number of widely expressed Rho family GTPases. Ligand-gated association between individual GEFs and GAPs with guidance cue receptors is one mechanism by which specificity is imparted to signaling pathways activated by various guidance cues as reviewed in (Mueller et al 1999)

\subsubsection{Guidance cues}

Neurons are guided by numerous molecular components ranging from membrane receptors to diffusible proteins and small molecular lipid carrier. Each guidance molecule initiates neuronal growth cone guidance with the specialized signaling network, which enables the proper wiring of neurons from different regions with its functional target. Guidance molecules are classified upon the functional response at a specific time and at specific location. The guidance cue can either be attractive or repulsive at specific location and can be completely the reverse at a different location depends on the signaling intermediate it encounters. They range from intracellular soluble to insoluble proteins and extracellular membrane anchored to trans-membrane proteins.

\subsubsection{Repulsive guidance cues}

Repulsive guidance cue are the molecules are that mediate the turns or collapse the growing axonal growth cones to the reverse direction of navigation to help the neurons to traverse and reach its final destiny. They range from small proteins to trans-membrane receptors that act in a coordinated fashion to help the neurons navigate 


\subsection{Lyso Phosphatidic Acid, 1-oleoyl-2-hydroxy-sn-glycero-3-phosphate (LPA)}

LPA is a serum-derived phospholipid that elicits a variety of cellular responses, including proliferation, chemotaxis, platelet aggregation, and smooth muscle contraction. LPA is generated from cleavage of cellular membrane phospholipids and thus exhibits some heterogeneity in its fatty acid composition. The palmitoyl and oleoyl fatty acid-containing phospholipids are the most prevalent in serum. Biological activity typically requires long acyl carbon chains (i.e., $\mathrm{C} 16$ or $\mathrm{C} 18)$.

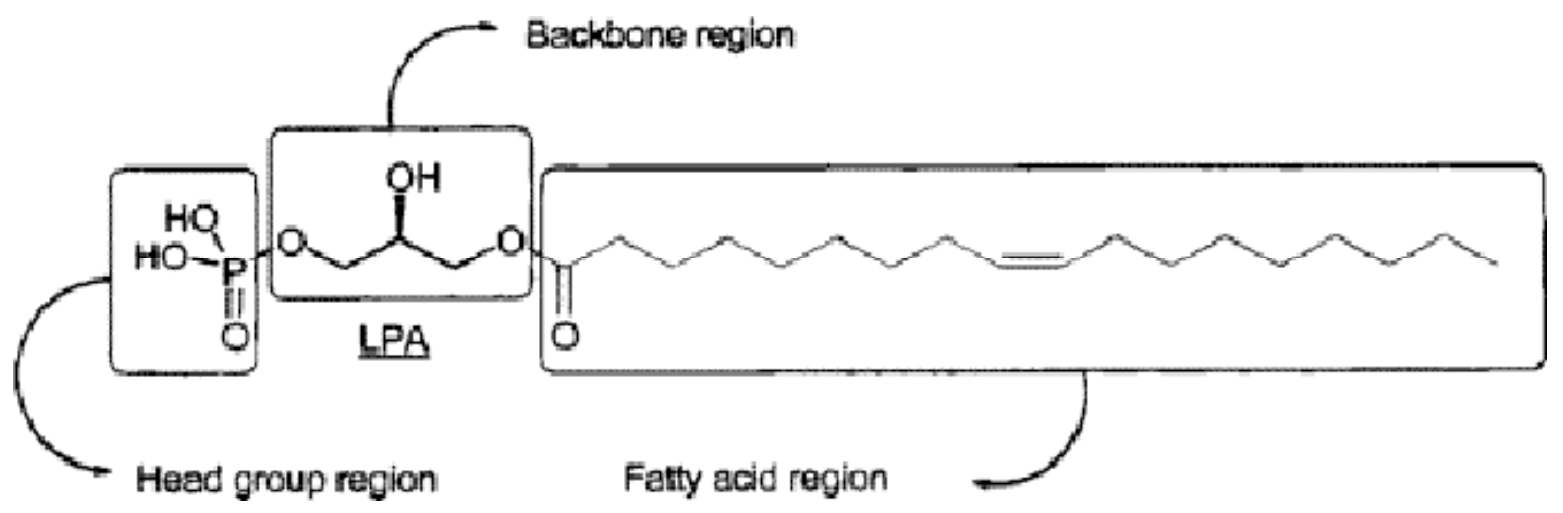

(Moolenaar. WH et al JBC 1995)

The actions of LPA are of wide range, and completely different among targeted cell types (W.H. Moolenaar et al, G. Tigyi et al 1995). In nervous tissues, (Jalink et al. and Tigyi et $a l$.$) , it was demonstrated that LPA induces morphological changes, such as growth cone$ collapse and neurite retraction. These activities are possibly related to the biological functions in neural morphogenesis, synaptic remodeling and regeneration failure after nerve injury. According to Jalink et al., the morphological responses are similar to the reactions 
induced by serum derived protease, thrombin. They also reported that LPA signaling is mediated by $\mathrm{G}$ protein coupled receptors and several second messenger systems involving small molecular weight G protein, Rho, calcium ions and protein kinases. Suramin, a polyanionic anti-parasite drug had been described as a LPA receptor antagonist by Van Corven et al. LPA causes growth cone collapse in primary cultured chick neurons. This action was dose dependent and the potency was almost identical in three different neuron types, dorsal root ganglion neurons, retinal neurons, and sympathetic ganglion cells. Fifty percent of growth cones were collapsed by $10^{-6} \mathrm{M}$ lysophosphatidic acid. The growth cone collapse started within 2 min after lysophosphatidic acid exposure and no homologous desensitization was observed. However, this action was reversible and not toxic to the neurons. Suramin, known as an antagonist to lysophosphatidic acid, which by itself had growth cone collapsing activity against, cultured primary neurons. This study demonstrated that LPA inhibit neurite growth of primary cultured neurons reversibly like in tumorderived cells. This result strongly supports the hypothesis that LPA acts as an inhibitory factor of neurite growth after nerve injury in vivo.

\subsection{Semaphorins and Neuropilins}

The semaphorins are a large group of axonal guidance molecules consisting at least of 30 different members (Chen et al 1997). Conserved in invertebrates and vertebrates, they serve as repulsive guidance cues, influencing growth cone guidance not only in a contactdependent way but also from a distance, as long-range chemo-repulsive cues (Messersmith et al 1995, Sheperd et al 1997, Varela-Echavarria et al 1997). In vivo data from 
grasshoppers and the presence of motifs known to promote neurite outgrowth has suggested that some members might function as attractive or permissive guidance cues (Inagaki et al 1995, Püschel et al 1995, Adams et al 1996, Kolodkin 1996, Mark et al 1997, JTW Wong et al 1997), although a direct demonstration of a positive effect remains to be obtained.

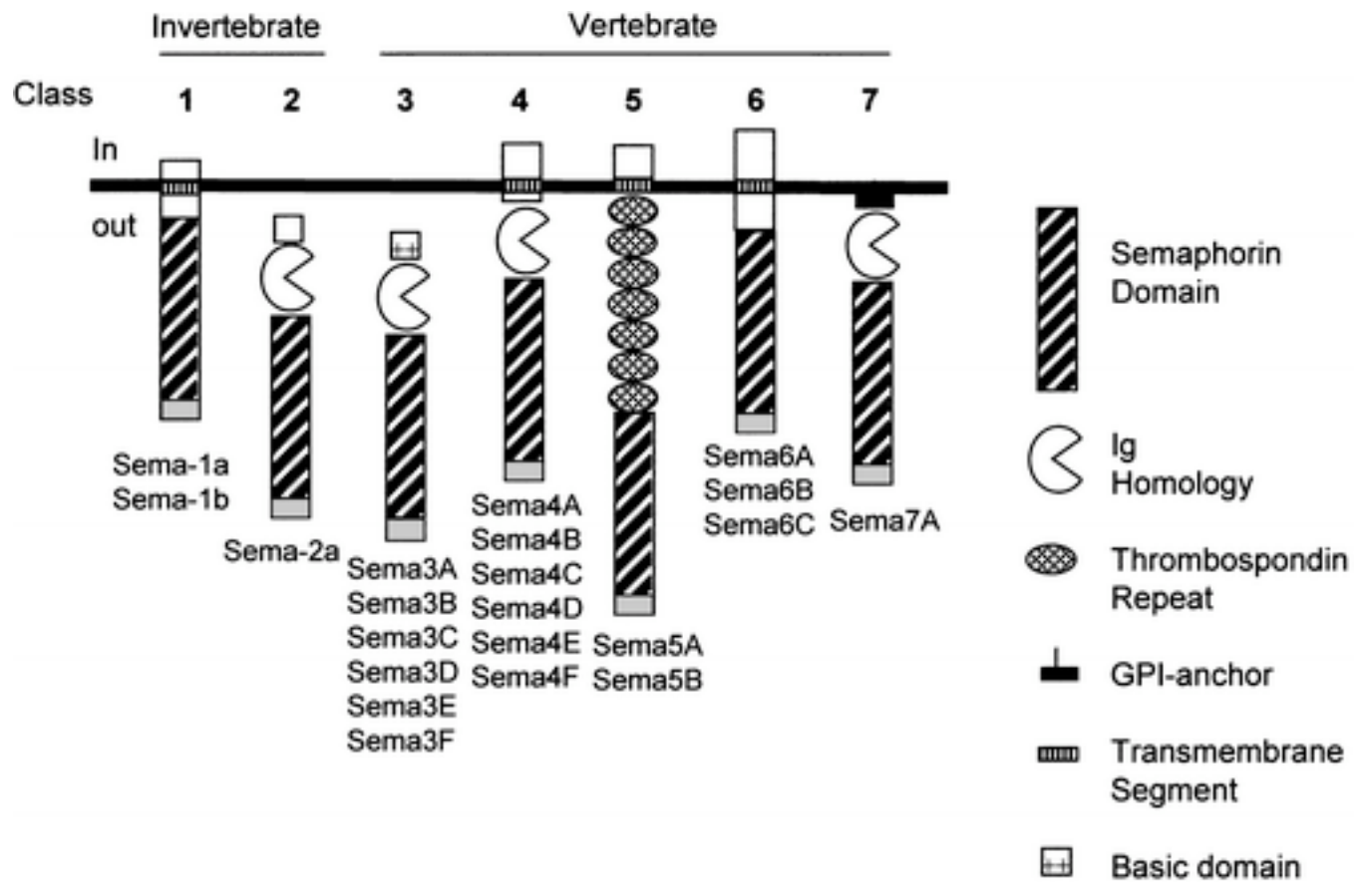

(Mueller BK et al Annu Rev Neuroscience, 1999)

The transmembrane semaphorin G-sema I (formerly fasciclin IV) was implicated in axonal guidance in the grasshopper limb bud (Kolodkin et al 1992). A first indication of a repulsive action came with the cloning of the first molecule inducing growth cone collapse (Luo et al 1993). Using growth cone collapse as an in vitro assay, membrane fractions from brain were purified and a molecule, collapsin-1 (formerly collapsin), was cloned that was extremely potent in inducing collapse: $10 \mathrm{pM}$ induced collapse of $50 \%$ of sensory growth cones. Collapsin-1 bound to beads induced turning of sensory growth cones often after filopodial contact alone and without inducing full collapse (Fan \& Raper 1995). Collapsin- 
1, a glycoprotein of $100 \mathrm{kDa}$ is extensively glycosylated and has no transmembrane domain, although it has a highly basic region near its $\mathrm{C}$-terminal end. A C2 immunoglobulin (Ig)-like domain is also found, and the only known protein with sequence homology in the N-terminal region was fasculin IV (Luo et al 1993). Based on sequence comparisons of this domain and structural similarities, semaphorins were grouped into six classes, with classes I, IV, V, and VI containing transmembrane forms and classes II and III secreted forms (Mark et al 1997, Kikuchi et al 1997).

\subsection{Neuropilins}

All classes of semaphorin have its binding partner in an equally large receptor family. The first candidates of receptors have recently been described, Using a COS cell expression and cloning approach, two groups independently identified a transmembrane protein called neuropilin as a collapsin-1/semaphorin III/D receptor (Neuropilin, initially identified by H Fujisawa and colleagues as a cell surface protein called A5, was suggested to be involved in axonal growth and guidance because of its expression on specific axon populations (Neuropils) in the nervous systems of Xenopus laevis, chicken and mouse embryos (Takagi et al 1991, Fujisawa et al 1997). Neuropilin has been shown to promote neurite outgrowth in vitro (Hirata et al 1993), after transfection of fibroblast-like cells (L cells) induced aggregation in cell aggregation assays. In these experiments, neuropilin acted as a heterophilic calcium-independent cell adhesion molecule, binding via its b1/b2 domains to molecules on the surface of L cells (Fujisawa et al 1997). Overexpression of neuropilin in 
mice driven by a beta-actin promoter resulted in defasciculation of nerves and ectopic branching (Kitsukawa et al 1995).

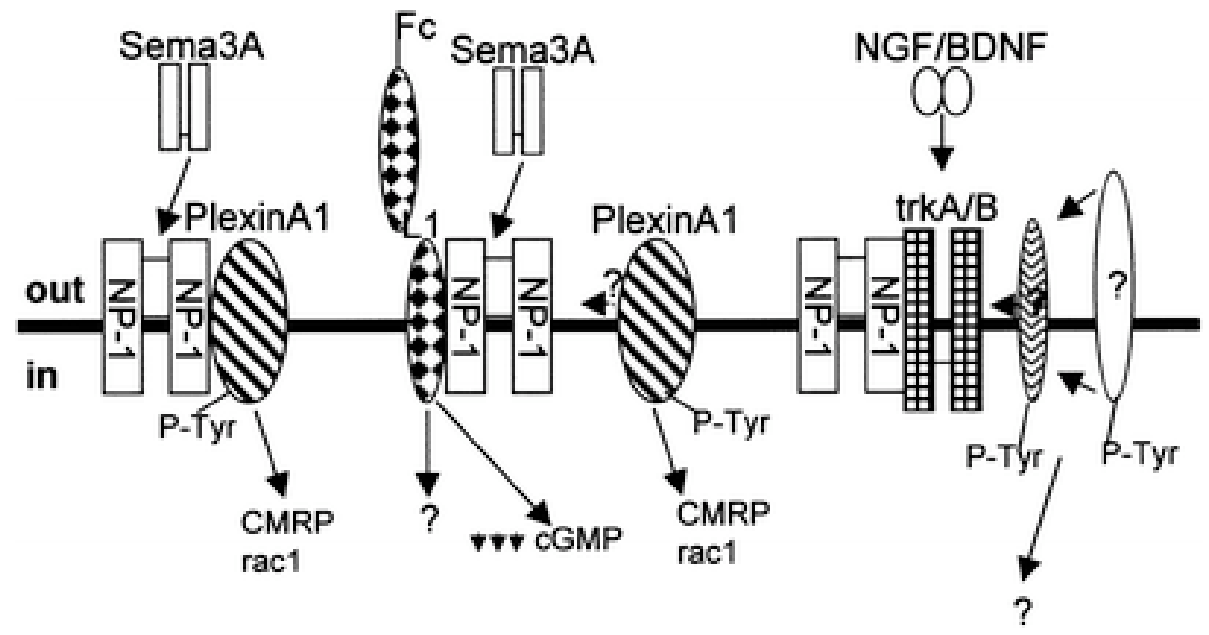

(Mueller BK et al Annu Rev Neuroscience, 1999)

\subsubsection{Attractive Guidance Cues}

\subsection{Netrins and Their Receptors}

The netrins are a family of proteins exerting attractive and repulsive effects on growing axons. Members of this family were found independently in invertebrates and vertebrates. A screen in C. elegans for mutants exhibiting defects in cell and axon migrations resulted in the discovery of more than 30 genes involved in these processes (Brenner 1974). Later it was shown that three genes, called unc-5, unc-6, and unc-40 (for "uncoordinated," indicating uncoordinated movement exhibited by mutants), are involved in circumferential guidance and migration of axons and cells along the dorsoventral axis (Hedgecock et al 1990, Ishii et al 1992, Wadsworth et al 1996, Hedgecock \& Norris 1997). In principle, the corresponding molecules could act as guidance cues or guidance receptors, or they could 
exert other functions. In a biochemical approach, designed for the detection of guidance molecules, M Tessier-Lavigne and coworkers, searched for a floor plate derived chemoattractant and succeeded in purifying from embryonic chicken brains, proteins called netrin1 and netrin-2. Netrin-1 and netrin-2 are expressed in the floor plate and ventral spinal cord, respectively, and attract commissural axons of the embryonic spinal cord (Kennedy et al 1994, Serafini et al 1994)

\subsection{Local protein turnover in growth cone guidance}

Positive guidance can either allow navigation for positive signals or inhibit for negative signals within growth cones, negative guidance inhibits the navigation or allow the positive turning of the growth cones to the signaling intermediate. Axonal growth cones often travel a long distance with a high degree of autonomy from the cell soma and it can even survive and grow in dissociated culture after severing from cell body (Shaw and Bray, 1977), maintains their unique properties similar to intact neurons (Guthrie et al., 1989). This suggests that the growth cone steering is likely mediated by local mechanisms independent of the cell soma; in agreement, retinal axons navigate correctly after soma removal (Shaw and Bray, 1977).

Two important properties of growing axons are their ability to navigate far from the cell body and their ability to change responsiveness to extracellular cues as they grow toward and reach their ultimate target, since growing axons in vivo make rapid decisions, sometimes at large distances from their soma as they encounter new molecular territories 
along the pathway (Alvarez et al., 2000), During growth and establishing connections growth cones have to respond to their signal sequences in an immediate fashion ranging from seconds to minutes. When these responses are compared to the distance traveled by axons from the cell body and the speed of molecules transported along the long axon in response to environmental stimuli, it is an impossible task that the growth cone responds to the guidance cue in an immediate time interval.

This insight suggests the involvement of local protein turnover in the axon and growth cone as a mechanism for growth cone navigation and wiring of network between and within neurons. Proteins are transported along the axons from the cell body by the slow and fast axonal transport. The proteins like cytoskeletal and cytosolic are primarily transported by slow axonal transport could possibly take days and years to reach the terminal of long axon (reviewed by Hirokawa et al., 1997). This provides the argument that how proteins last this extended journey and supplied to distal tip of the axon. Evidence has accumulated indicating that axons contain specific mRNAs and ribosomes and can synthesize cytoskeletal proteins and some other proteins. It remains unclear whether local protein synthesis could supply the cytoskeletal proteins and other slow-transported proteins that are required for the maintenance, plasticity, and regeneration of long axons. The abovementioned roles of local protein turnover machineries like transport; synthesis, degradation and chaperone assisted folding of protein in axon are discussed in detail below, will provide the readers with understanding of functional roles of individual machineries of protein folding in axonal guidance and development of nervous system. 


\subsubsection{Axonal transport mechanism in growth cone guidance}

Intracellular movement is the key to the functioning of eukaryotic cell. In the complex macromolecular environment of the cytoplasm, proper functioning is impossible without the constant movement of intracellular components from place to place. In recent years we have observed a change in our understanding of the extent of these movements inside cells and their underlying molecular mechanisms.

We now know that intracellular transport is coordinated by a diverse array of molecular motor proteins that bind specific cargoes and direct them in a particular direction along cytoskeletal tracks (Vale and Milligan, 2000). Moreover, the cargoes themselves are far more diverse than previously imagined, including every type of membranous organelle and species of transport vesicle, as well as nonmembranous cargoes such as cytoskeletal polymers, cytosolic protein complexes, ribosomes, and messenger RNAs.

\subsubsection{Slow versus Fast axonal transport with examples}

The difference in the rate of fast and slow axonal transport has long been assumed to indicate that membranous and nonmembranous cargoes move by fundamentally distinct mechanisms, but direct observations on the movement of these cargoes in living cells now indicate that they are all transported by fast motors and that the principle difference between fast and slow transport is not the rate of movement per se, but the manner in which the movement is regulated. Various proteins and components like, cytoskeletal proteins, 
membrane receptors, organelles, and soluble transmembrane protein were reportedly transported along axons by slow or fast mode of transport, as explained with examples of neurofilament and $\alpha$-synuclein

\subsection{Neurofilament-M subunit}

Recently the movement of neurofilaments and microtubules has been observed in axons, and these observations indicate that the solution to the slow axonal transport controversy is relatively simple. Cytoskeletal polymers do move in axons, but their movements are not slow after all. Both neurofilaments and microtubules move at fast rates, compared to the rate of movement of membranous organelles, but the average rate of movement is slow because the movement is both infrequent and bidirectional (Roy et al., 2000; Wang et al., 2000; Wang and Brown, 2001).

\subsubsection{2. a-Synuclein}

Synucleins are abundant nerve terminal proteins of hitherto unknown function. In diseases with Lewy bodies, human $\alpha$-synuclein concentrates in these lesions in the cell body and mutations in led to heritable Parkinson's disease with Lewy bodies. This indicates that changes in the normal metabolism and axonal transport of $\alpha$-synuclein are perturbed in these diseases. Recent work (Jensen. PH et al 1999) show that synucleins are transported by fast anterograde and retrograde transports and colocalize with synaptophysin and SNAP-25 around the lesion sites. 


\subsubsection{Protein synthetic machinery in the growth cone guidance}

Vertebrate growth cones possess the machinery necessary for protein translation (Bassell et al. 1998 and Tennyson 1970) and can translate proteins locally (Crino and Eberwine 1996 and Davis et al. 1992). Developing dendrites possesses multiple different mRNAs (Crino and Eberwine, 1996) and surprisingly, inhibiting the protein synthesis in hippocampal neurons blocks synaptic long-term depression (LTD) on the rapid timescale of 5 min (Huber et al., 2000), In principle, one mechanism to regulate axon responsiveness could be to synthesize proteins, such as cell surface receptors, within the distal segment of the axon after reaching an intermediate target. While cytoplasmic poly-adenylation, local translation has been studied extensively as a mechanism for synapse regulation in dendrites (Richter 1999; Martin et al. 2000; Wells et al. 2000; Job and Eberwine 2001 and Steward and Schuman 2001), it is assumed that the vertebrate axons are not capable of protein synthesis, based on studies that failed to detect ribosome's, and the ability of axons to transport proteins from the cell body (Tanaka and Sabry 1995; Alvarez et al. 2000; Lodish et al. 2000 and Schwartz and DeCamilli 2000).

Many studies over a period of four decades based on labeled amino acid incorporation into isolated axon preparations, inhibitors of translation, and detection of various components of protein translation machinery have led to increasing interest in the idea of RNA translation by vertebrate axons (Koenig 1967; Bassell et al. 1998; Eng et al. 1999; Koenig et al. 2000 and Campbell and Holt 2001; reviewed by Alvarez et al., 2000). However the question of whether vertebrate axons could export locally synthesized proteins such as guidance 
receptors to the cell surface remains unsolved. In the case of vertebrate axons, it was strongly believed for several decades that they receive all their proteins by anterograde transport from the cell body and are not capable of translation (Tanaka and Sabry 1995; Lodish et al. 2000 and Schwartz and DeCamilli 2000).

Protein translation in dendrites is now well established as a mechanism for synapse regulation (Martin et al. 2000; Wells et al. 2000; Job and Eberwine 2001 and Steward and Schuman 2001). Translation has also been recognized to occur in large axons of invertebrates such as Aplysia, although these have dendrite-like features and are considered to differ in this regard from vertebrate axons (Martin et al. 2000 and Spencer et al. 2000).

Signaling molecules like Semaphorin 3a and Netrins in addition, were shown to regulate the neurite extension by controlling the gene expression through mitochondria and p42/p44 MAPK mediated signaling cascade in PC12 and hippocampal neurons (Schwamborn JC et al 2004). An increased production of mRNAs for beta-actin, plexin A1 and Crmps are well documented and these studies imply that protein synthesis is a key player in regulated activity of growth cone navigation. Proteins like neurofilament subunits, synaptophysin, $\alpha-$ spectrin, filamentous actin, and ezrin are well documented for their significant synthetic in neuronal presynaptic compartment, (Brown. A et al 2001. Wheeler, TC et al 2002., Sangerman. J et al 2001) 


\subsubsection{Protein degradation machinery in the growth cone guidance (Ubiquitin Proteasome System (UPS))}

\subsubsection{Ubiquitin}

Ubiquitin is a member of a family of small proteins that are structurally similar and have the unusual property of forming a stable chemical bond with other proteins. Ubiquitin carries a C-terminal glycine residue with a carboxyl group that forms an isopeptide bond with the $\varepsilon$-amino group of lysine residues, or less commonly, with the $\alpha$-amino group at the $\mathrm{N}$-terminus of a substrate protein. The conjugation of ubiquitin to a protein can regulate a protein in different ways. Known or suspected mechanisms of ubiquitin action include altering protein stability, location, binding partners, or physical conformation.

Ubiquitin modifies proteins involved in many cellular functions (Glickman \& Ciechanover 2002, Hicke \& Dunn 2003, Muratani \& Tansey 2003, Peng et al. 2003). Lys48-linked polyubiquitin chains have been studied extensively, and they regulate nuclear, cytosolic, and endoplasmic reticulum membrane proteins by targeting these proteins for degradation by the $26 \mathrm{~S}$ proteasome (Glickman \& Ciechanover 2002). Lys63-linked chains are known to regulate DNA repair, signal transduction, and endocytosis and are likely to control other basic cellular processes as well (Fisk \& Yaffe 1999, Galan \& Haguenauer-Tsapis 1997, Hoege et al. 2002). Mono-ubiquitination also serves as an important regulatory signal for many cellular functions like down regulation of receptors (Schnell \& Hicke 2003). 


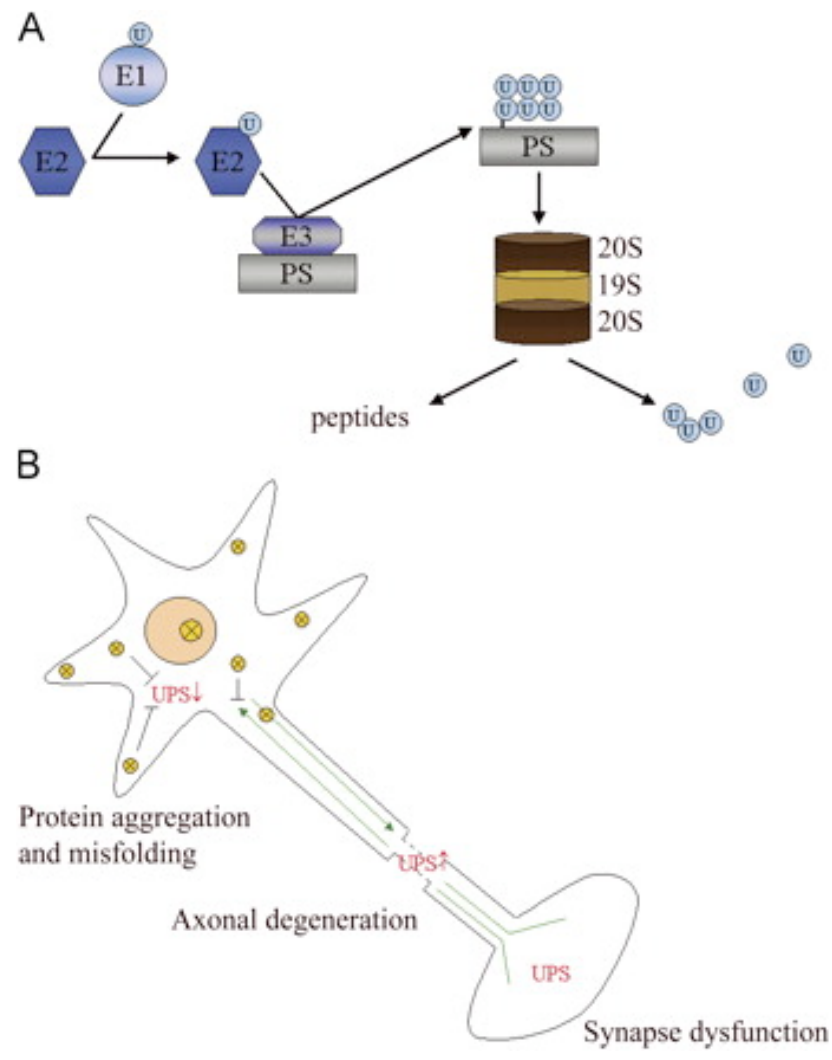

Korhonen. L et al JCB 2004

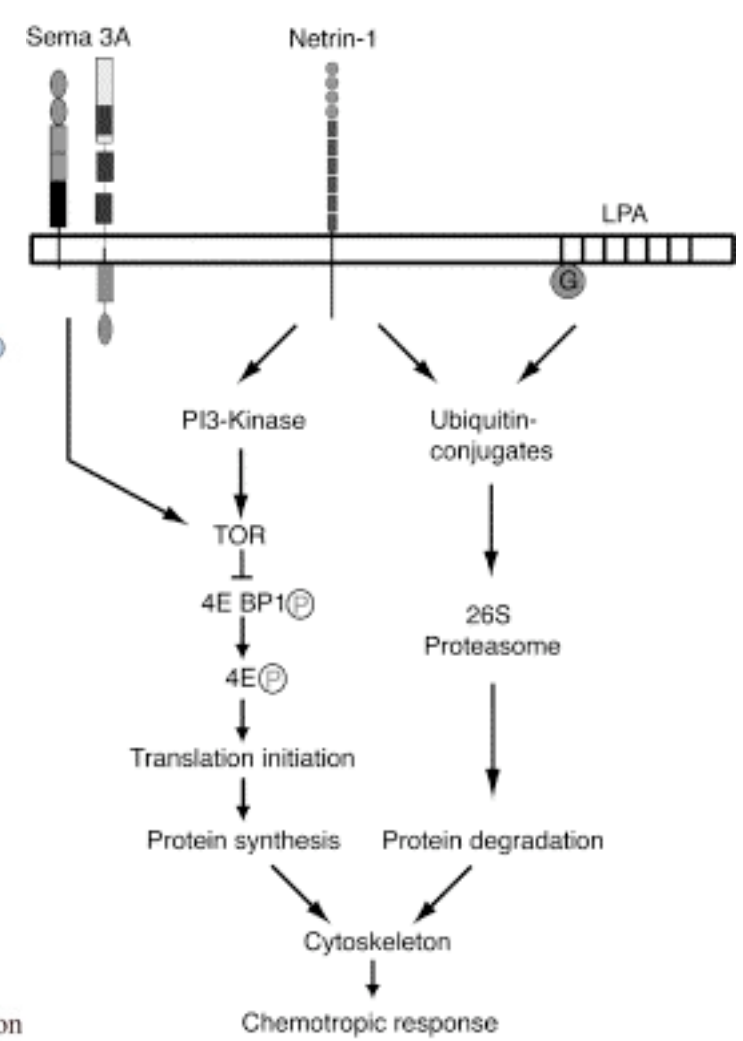

Campbell. DS \& Holt.C et al Neuron 2001

\subsubsection{Ubiquitination in the nervous system}

Ubiquitin and the ubiquitination machinery are potent regulators of protein stability, localization, and activity. Their function in the nervous system has been investigated for many years, although the primary focus has been on the role of ubiquitination in neurodegenerative diseases (reviewed in Gassen and Lee et al 2003, Mayer et al 2002). Recently, a number of studies have linked ubiquitin modification to the development and function of synapses in the "healthy" nervous system. In fact, ubiquitin's role in neurodegeneration may result, in part, from the impairment of ubiquitin-dependent regulation of normal neuronal physiology. 
Ubiquitination and the proteasome system together can accomplish the recycling of excess protein in the axonal growth cone compartment and serves an essential function in maintaining the integrity of synapse. Wiring the developing brain is an essential part of building a functional nervous system. Establishing the appropriate connections is a complex, multistep task (.Huber et al 2003, Yu and Bargmann 2001). A series of recent observations confirms that the ubiquitin and the proteasome are important regulators of neuronal connectivity, axon guidance, axonal pruning, synapse formation and growth (Reviewed in Di Antonio.A et al 2004).

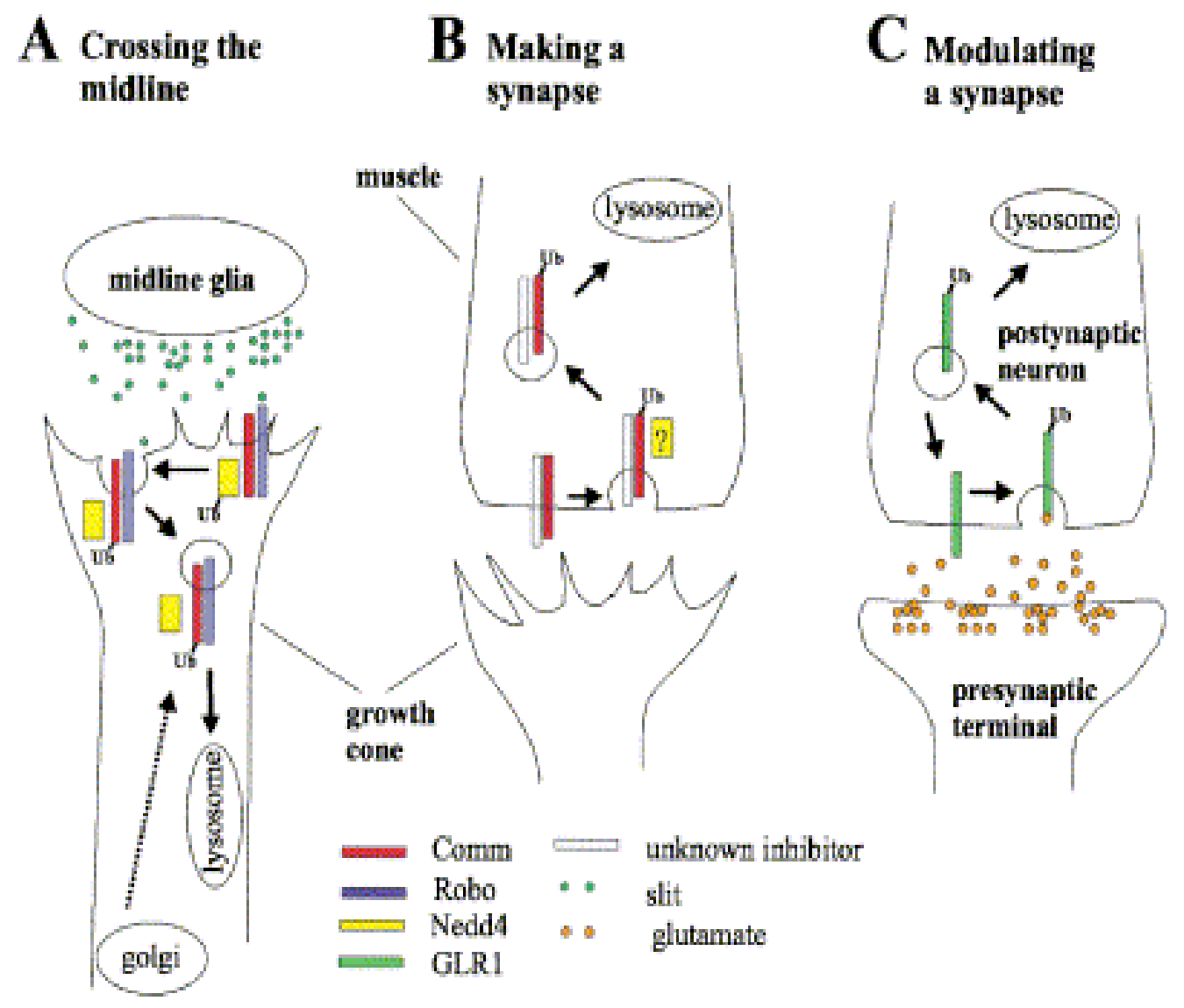

(Di Antonio.A et al Annul Rev Neuroscience 2004).

Ub-Ubiquitin 


\subsubsection{Proteasome mediated protein degradation in neuronal axons}

The presence of the protein degradation machinery in the growth cone has been a debated issue that gained a momentum by the evidence of protein ubiquitination machinery and proteasome system by the work, which represent level of protein recycling in neuronal growth cones (sangerman and Goodman 2001; Gianluca and Letourneau 2002), and the effect of local response of proteasome and protease inhibitor on the growth cone (Campbell and Holt 2001). In addition to key players like kinases, phosphatases, small G proteins, cyclic nucleotides, and intracellular calcium, the protein translation machinery (Doherty et al. 2000, K orey and Van Vactor 2000, Brittis et al, Campbell and Holt 2001), are the components that regulate growth cone behavior. The study on the drosophila mutant bendless shed light on to the involvement of ubiqutin in regulating neuronal connectivity (Thomas and Wyman 1984), it was reportedly identified that bendless was an E2 ubiquitinconjugating enzyme (Muralithar and Thomas 1993, Oh et al. 1994). By using repulsive guidance cue in the giant fibre the other group has demonstrated that changing levels of semaphorin signaling give a similar phenotype to that of bendless mutant (Murphey et al. 2003) and was appreciated for the idea of bendless may be regulating the levels of key signaling molecules on the growth cone (Muralithar and Thomas 1993, Oh et al. 1994). These seminal findings leadingly paved the way for field of developmental neurobiology to ubiquitination and interrelating this link with other guidance molecules like Ephrins, Semaphorins, Netrins and Slits. A recent work in Xenopus retinal growth cones also demonstrates that ubiquitin dependent proteasomal degradation is a conclusive answer for

local protein turnover as the mechanism of growth cone navigation (Campbell and Holt 
2001), shows that the growth cones of Xenopus retinal axons in culture contain ubiquitin, the E1 ubiquitin activating enzyme, and proteasome components.

\subsubsection{Protein folding machinery in the growth cone guidance}

\subsubsection{Molecular chaperones OR Heat Shock Proteins (HSPs)}

Chaperones are known to be a large group of unrelated protein families, which play a role in stabilizing unfolded proteins, unfold them for translocation across membranes, for degradation, and to assist in their correct folding and assembly. Most, but not all, heat shock proteins are molecular chaperones. Molecular chaperones bind and stabilize proteins at intermediate stages of folding, assembly, translocation and degradation. Heat shock proteins have been classified by molecular weight, for example, Hsp70 for the 70-kDa, heat shock protein. Hsp70 null mutants of E. coli cannot grow at elevated temperature. Hsp100 null fruit flies lose their capacity for acquired thermo tolerance. Heat shock proteins are among the most well conserved proteins known. Amino acid sequences for Hsp70s of $E$. coli and man are almost $50 \%$ identical.

In the nervous system, Hsp70 overexpression in cultured hippocampal (Beaucamp N., et al 1998, Fink S.L. et al 1997) and peripheral (Uney JB et al 1993. Mailhos C and Uney JB et al 1994) neurons and glia (Uney JB et al 1994) similarly are protected against insults such as heat shock and metabolic stresses. Hsp70 expression can also be suppressed with antisense oligonucleotides that inhibit transcription (Sato et al 1996). Using this approach, 
Sato et al found that the protection from induced tolerance was reversed with Hsp70 blockade using antisense oligonucleotides. Hsp70 overexpression is not protective in all instances. Using a defective herpes simplex virus (HSV) vector (Fink et al 1997) showed that Hsp70 overexpression protected cultured hippocampal neurons from severe heat shock, but failed to protect against direct application of glutamate or 3-nitro propionic acid (3-NP), a mitochondrial toxin. From the studies of (Wagstaff, et al 1999), it was showed that Hsp70 overexpression protected cultured peripheral neurons from thermal and simulated ischemia, but not apoptotic stimuli, which in turn suggest a link to other signaling cascade with HSP70.

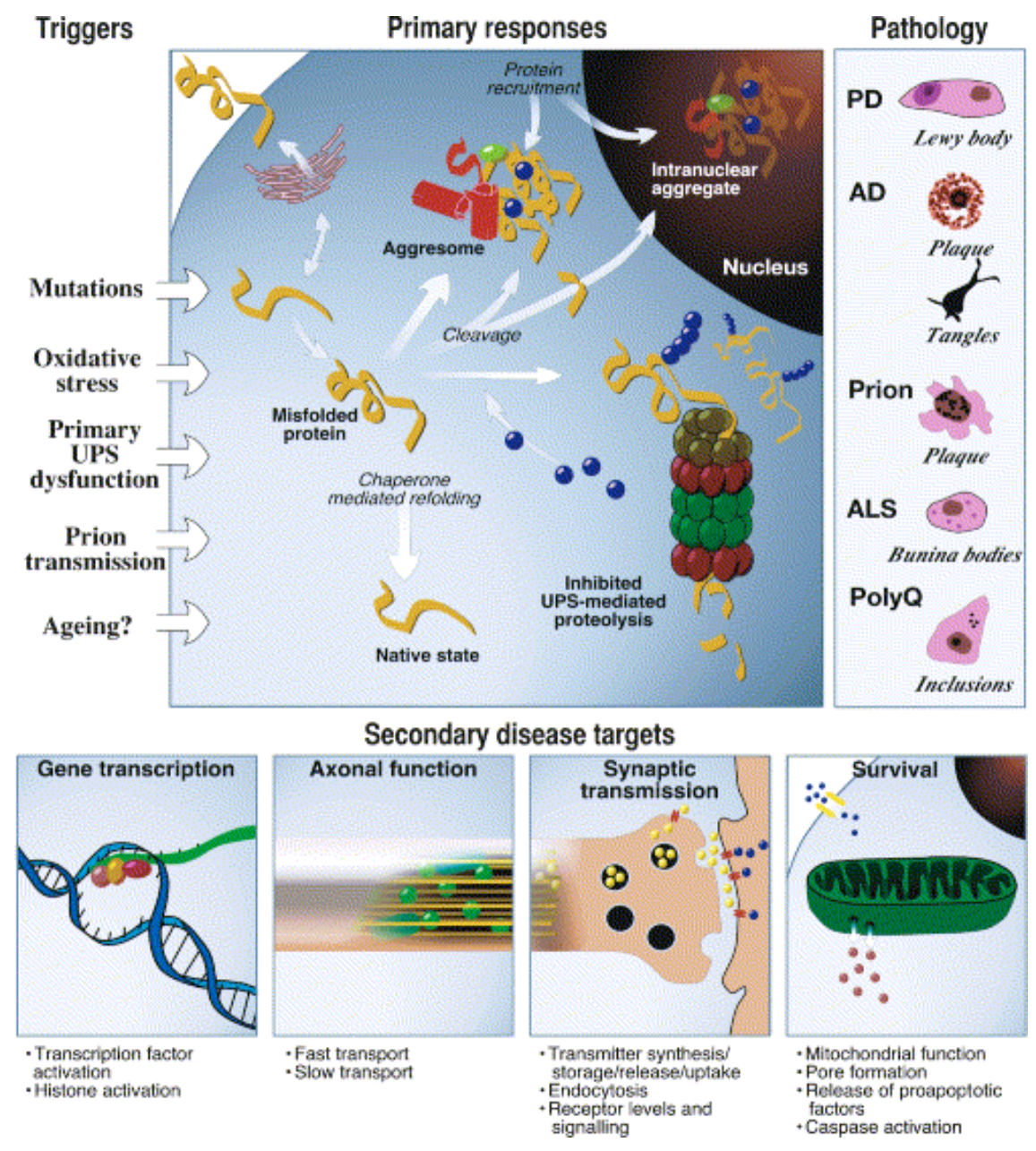

(Ciechanover et al Neuron 2003) 


\subsubsection{BAG-1 (Bcl-2-associated athanogene)}

BAG1 is a multifaceted protein implicated in the modulation of a large variety of cellular processes and was identified as a binding partner of anti-cell death protein Bcl-2 a known regulator of apoptosis (Takayama et al 1995). BAG1 is known for its multitude of functions ranging from transcriptional regulation to control of cell migration. A common element of the remarkable functional diversity of BAG-1 is through the interaction with molecular chaperones of the Hsp70 family (reviewed in Takayama and Reed 2001; Doong et al 2002). BAG-1 functions as a nucleotide exchange factor for mammalian cytosolic Hsc70, thereby triggering substrate unloading from the chaperone and has been proposed to promote cell survival by association of BAG-1 with the proteasome, which suggests a role in coordinating chaperone and degradation pathways. BAG-1 is an ubiquitin domain protein that links the molecular chaperones Hsc70 and Hsp70 to the proteasome (Connell, P Jiang, J, Demand, J, Murata, S 2001).

During proteasomal sorting BAG-1 can cooperate with another co-chaperone, the carboxyl terminus of Hsc70-interacting protein CHIP. CHIP was recently identified as an Hsp70and Hsp90-associated ubiquitin ligase that labels chaperone-presented proteins with the degradation marker ubiquitin. Ubiquitylation of BAG-1 is strongly stimulated when a ternary Hsp70·BAG-1·CHIP complex is formed (Alberti S., et al 2002). Complex formation results in the attachment of an atypical polyubiquitin chain to BAG-1, in which the individual ubiquitin moieties are linked through lysine 11 . The non-canonical polyubiquitin chain does not induce the degradation of BAG-1, but it stimulates a 
degradation-independent association of the co-chaperone with the proteasome. The cochaperone BAG1 binds and regulates $70 \mathrm{kDa}$ heat shock proteins (Hsp70/Hsc70) and exhibits cytoprotective activity in cell culture models. Bag1 protein is expressed early in neurogenesis in vivo and is capable of modulating neuronal cell survival and differentiation at least in part from a nuclear location.

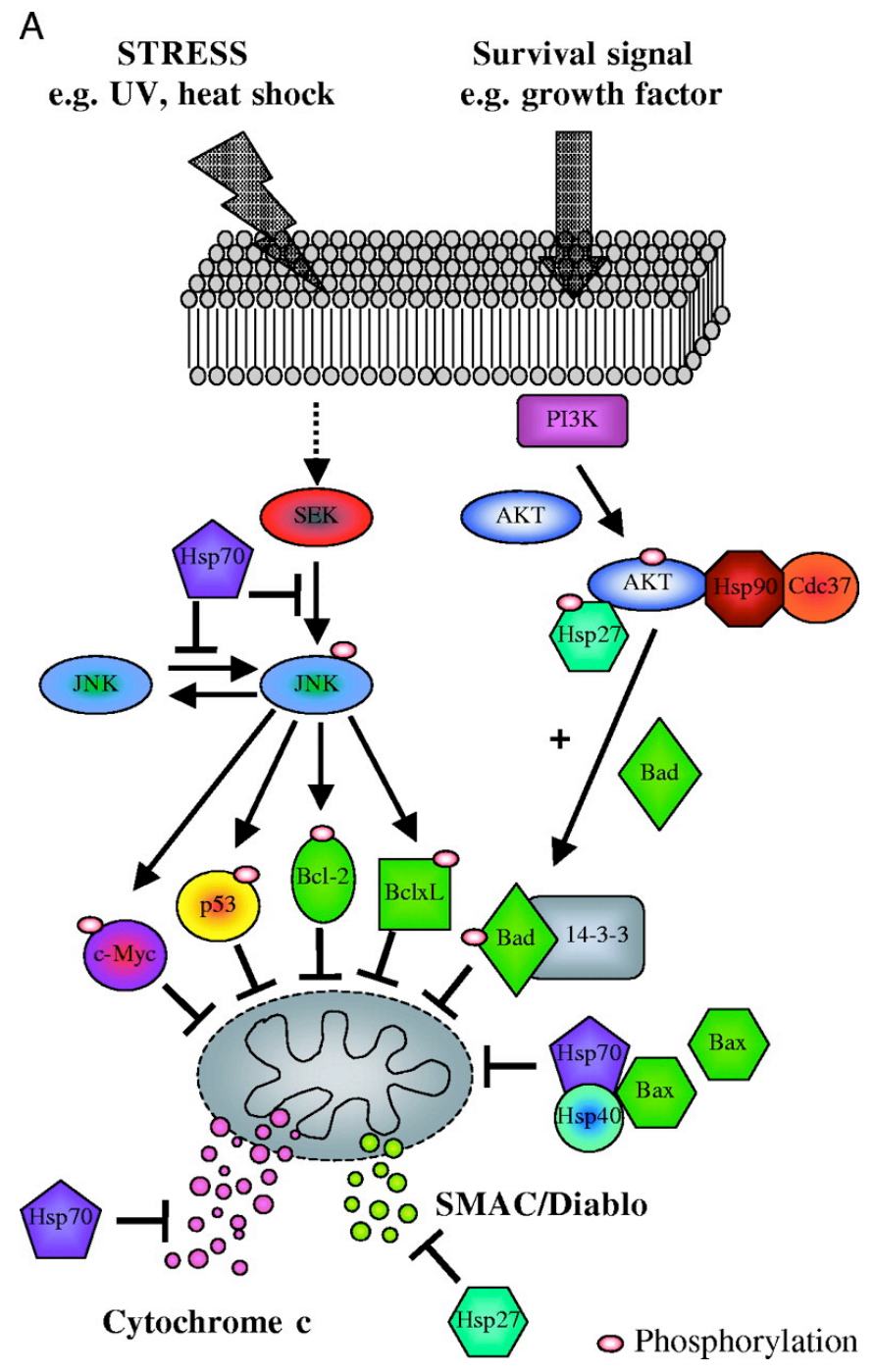

Beere. HM JCS 2004 
Recently it was demonstrated that BAG1 expression is induced during neuronal differentiation in the developing brain (Kermer P and Reed .JC, et al 2003). However, the in vivo effects of BAG1 during development and after maturation of the central nervous system have never been examined. Experiments with transgenic mice over-expressing BAG1 in neurons, revealed resistance to glutamate-induced apoptotic neuronal death of cultured cortical neurons, while brain development was essentially normal. Moreover, in an in vivo stroke model involving transient middle cerebral artery occlusion, BAG1 transgenic mice demonstrated decreased mortality and substantially reduced infarct volumes compared to wild-type littermates. Interestingly, brain tissue from BAG1 transgenic mice contained higher levels of neuroprotective Hsp70/Hsc70 protein but not mRNA (Kermer .P and Reed .JC, et al 2002), suggesting a potential mechanism involving stabilization of Hsp70/Hsc70, The role of BAG1 in anti-apoptotic cascade and its neuro-protective activity against stress related proteins will be discussed in the following results chapter.

\section{Schematic model for growth cone protein turnover machinery}

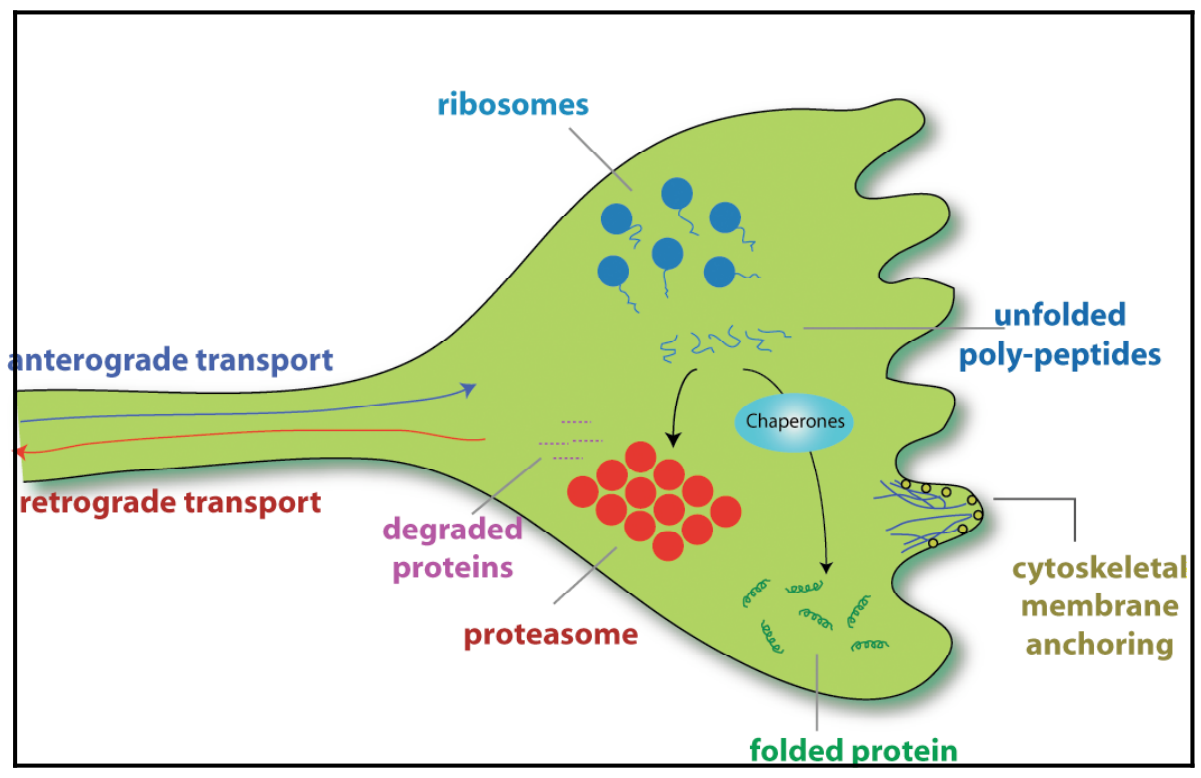




\subsection{Local protein turnover as regulatory mechanism for growth cone guidance}

Based on the above-mentioned facts and argumentation, we would like to propose that the regulation of local protein turnover plays a key role in the growth and collapse of neuronal growth cones.

As argued above, proteins are transported along the axons in a rather slow rate, axonal transport can therefore not be sufficient to supply necessary proteins to the growth cone in a time frame required to explain the morphological changes in the growth cone. On the other hand, retrograde axonal transport could contribute to the rapid clearance of the proteins as the growth cones presents a small confined volume. Of course, we cannot exclude the anterograde delivery of proteins to the growth cones from a distal axonal pool. However, this optional mechanism cannot explain the continuous and repetitive morphological changes of the advancing growth cones as the distal axonal pool of proteins become depleted and cannot be replenished in time. Furthermore, this would mean that all possible necessary proteins are always present in this pool. As the presence of local protein synthesis has been established, this presents a more likely source for the rapid introduction of larger amount of specific protein to the growth cone. The presence of proteasome components in the growth cones suggests that proteins can be recycled and cleared, depending on need. 
As both of these mechanisms; ribosomal protein translation from axonally delivered mRNAs and the ubiquitin proteasome system, present tonically active machineries, increased clearance, production, or recycling rates of specific proteins demands the necessity for regulation of the protein cycle at the level of chaperones. Chaperones are positioned at the ideal location to control protein levels as their inactivity would cause newly produced nascent polypeptide chains to be shuttled to the proteasome directly, and their activity would lead to the folding and therefore functional stabilization of specific proteins. In order for this cycle to work properly in response to environmental cues and the metabolic status of growth cones, chaperones need to be regulatable other than through their synthesis. This regulation necessarily has to be embedded in the cellular signaling network in order to respond to external stimuli.

Acute changes in chaperone activity, thus produced, would lead to the desired changes in the composition of the local growth cone proteome. Given the morphological changes of the advancing growth cone, the proteins that are likely under control of these regulatory mechanism include, cytoskeletal associated and structural proteins. These will be briefly discussed in a later results section. I will next discuss the chaperone system and point toward the first indication that such signaling-depended regulation can occur. I will discuss the BAG1 protein as such a signaling node as it regulates HSP70 activity, signals downstream through the RAF kinase, ERK pathway, which is involved in differentiation, and is itself under regulatory control of an upstream anti apoptotic signaling network through its connection to the Bcl-2 protein. Disturbances in this tightly regulated cycle are also likely to be involved in neurodegenerative conditions, were aggregation of proteins 
like Poly-glutamine proteins, microtubule binding protein Tau, and $\alpha$-synuclein, lead to neurotoxicity in Huntingtin's disease, Alzheimer's disease, and Parkinson's disease respectively.

There is accumulating evidence that these protein aggregates inhibit the proteasomal function, lead to aberrant transcription of proteins, thereby upsetting normal cellular homeostasis. In fact, the expression or BAG1 protein is known to limit ischemic damage in stroke model systems. These pathophysiological implications will be discussed in a later section of the results chapter.

\subsection{Imaging techniques used-FRET and FLIM}

\subsubsection{Principle of FRET}

Fluorescence resonance energy transfer (FRET) is a distance-dependent physical process by which energy is transferred non-radiatively from an excited molecular fluorophore (the donor) to another fluorophore (the acceptor) by means of intermolecular long-range dipole-dipole coupling. FRET can be an accurate measurement of molecular proximity at angstrom distances (10-100 $\mathrm{A}$ ) and highly efficient if the donor and acceptor are positioned within the Förster radius (the distance at which half the excitation energy of the donor is transferred to the acceptor, typically 3-6 nm). The efficiency of FRET is dependent on the inverse sixth power of intermolecular separation (Förster, 1965; Clegg, 1996; Lakowicz, 
1999), making it a sensitive technique for investigating a variety of biological phenomena that produce changes in molecular proximity (dos Remedios et al., 1987).

\subsubsection{Requirement for FRET}

Technological advances in light microscopy imaging, combined with the availability of genetically encoded fluorescent proteins provide the tools necessary to obtain spatial and temporal distribution of protein associations inside living cells (Heim and Tsien, 1996; Day, 1998; Elangovan et al., 2002, 2003). The widely used donor and acceptor fluorophores for FRET studies come from a class of auto-fluorescent proteins, called GFPs. The spectroscopic properties that are carefully considered in selecting GFPs as workable FRET pairs include sufficient separation in excitation spectra for selective stimulation of the donor GFP, an overlap ( $>30 \%)$ between the emission spectrum of the donor and the excitation spectrum of the acceptor to obtain efficient energy transfer, and reasonable separation in emission spectra between donor and acceptor GFPs to allow independent measurement of the fluorescence of each fluorophore (Pollok and Heim, 1999).

\subsubsection{FRET imaging techniques and biological applications}

Whereas light microscopy initiated our understanding of cellular structure and the associated function, molecular biological studies over the past few decades have shown that cellular events, such as signal transduction and gene transcription, require the assembly of proteins into specific macromolecular complexes. Traditional biophysical or biochemical 
methods did not provide direct access to the interactions of these protein partners in their natural environment. Intensity-based imaging techniques applying the method of FRET microscopy (wide field, confocal, and multiphoton [MP]) were subsequently developed, facilitating the study of these interactions inside intact living cells (Periasamy, 2001). GFPbased FRET imaging methods have been instrumental in determining the compartmentalization and functional organization of living cells and for tracing the movement of proteins inside cells (Hanson and Kohler, 2001).
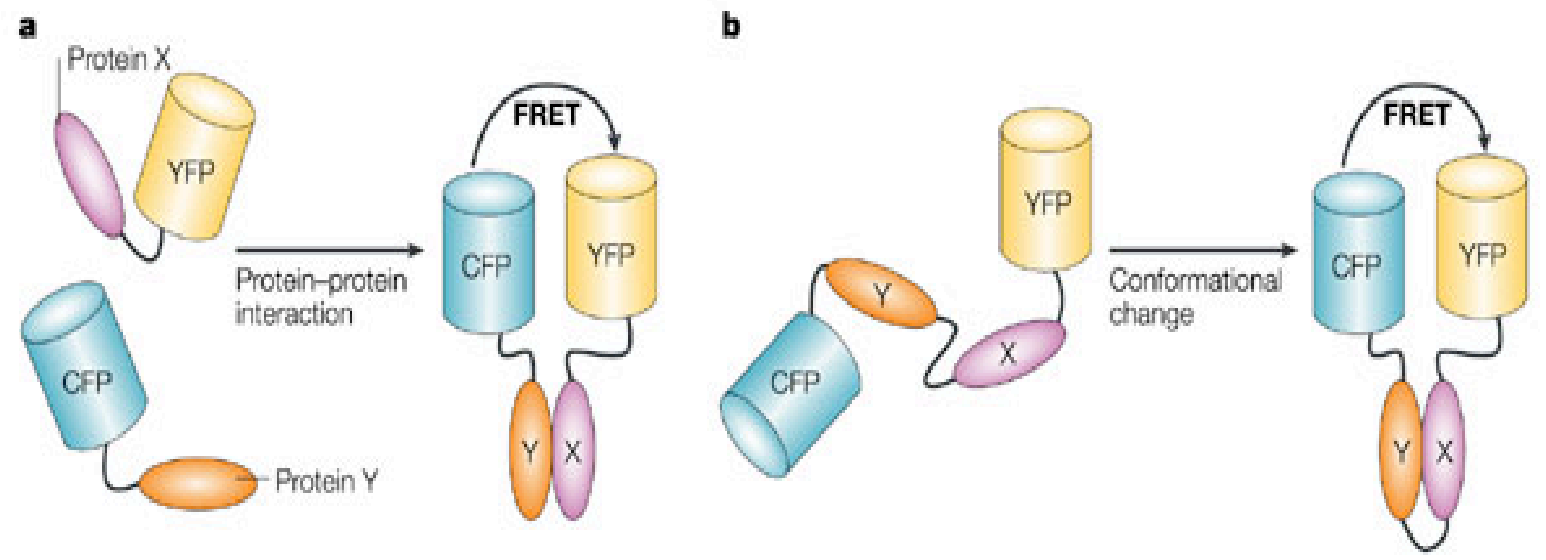

c

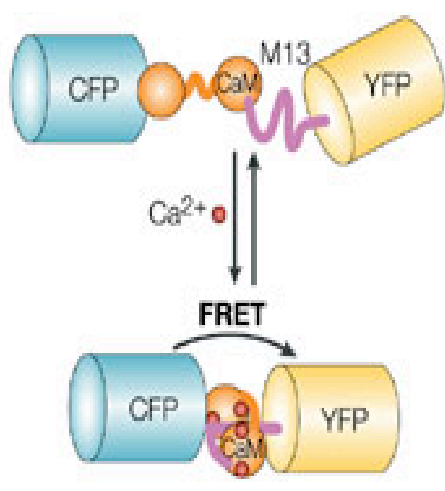

b

d

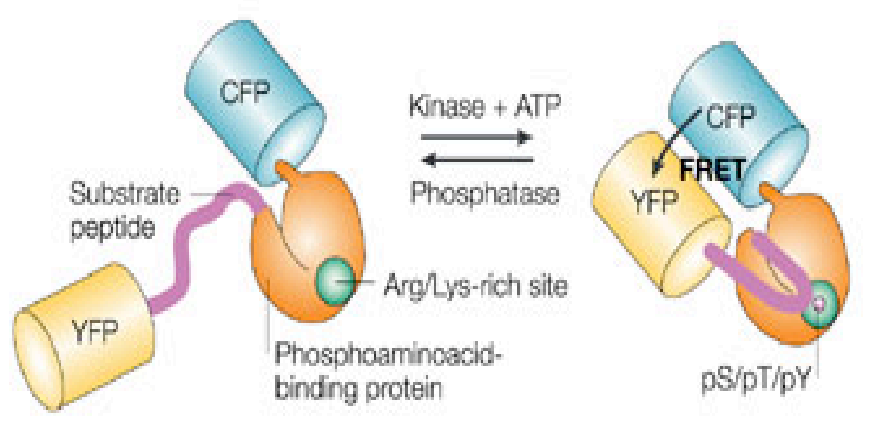

Nature Reviews | Molecular Cell Biology

New imaging technologies, coupled with the development of new genetically encoded fluorescent labels and sensors and the increasing capability of computer software for image acquisition and analysis, have enabled more sophisticated studies of protein functions and 
processes ranging from gene expression to second-messenger cascades and intercellular signaling (Roessel and Brand, 2002, Bunt et al). New fluorescent indicators have allowed the measurement of $\mathrm{Ca} 2+$ signals in the cytosol and organelles that are often extremely localized (Miyawaki et al., 1997) and nondestructive imaging of dynamic protein tyrosine kinase activities in single living cells (Ting et al., 2001).

\subsubsection{FLIM (Fluorescent Lifetime Imaging Microscopy)}

\subsubsection{Principle of FLIM}

The fluorescence lifetime is defined as the average time that a molecule remains in an excited state prior to returning to the ground state. For a single exponential decay, the fluorescence intensity as a function of time after a brief pulse of excitation light is described as

$I(t)=I 0 \exp (-t /)$

Where I0 is the initial intensity immediately after the excitation pulse. In practice, the fluorescence lifetime $(\tau)$ is defined as the time in which the fluorescence intensity decays to 1/e of the intensity immediately following excitation. Fluorescence decay is often multi exponential, leading to complex decay curves. The combination of lifetime and FRET (FLIM-FRET) provides high spatial (nanometer) and temporal (nanoseconds) resolution

(Bacskai et al., 2003; Elnagovan et al., 2002; Krishnan et al., 2003). The presence of 
acceptor molecules within the local environment of the donor that permit energy transfer will reduce the fluorescence lifetime of the donor. By measuring the donor lifetime in the presence and the absence of acceptor one can, in principle determine the distance between the donor and acceptor-labeled proteins.

\subsubsection{Frequency Domain FLIM}

Frequency domain FLIM uses sinusoidally modulated light source as a excitation light and senses the lifetime dependent demodulation and phase delay of the emission signal. Pulsed excitation can also be applied in the frequency-domain as it was used in the case of timedomain FLIM. Wide field microscopes are commonly used for lifetime detection in frequency domain FLIM (reviewed in Esposito. A et al, Curr prot in cell biology 2004).

\subsubsection{Time-domain FLIM}

In time-domain methods, pulsed light is used as the excitation source, and fluorescence lifetimes are measured from the fluorescence signal directly or by photon counting. Temporal resolution of the imaging modalities can be achieved by the technique of fluorescence lifetime imaging (FLIM). This technique monitors the localized changes in probe fluorescence lifetime and provides an enormous advantage for imaging dynamic events within the living cells. When combined with FRET, this approach provides direct evidence for the physical interactions between two or more proteins with very high spatial and temporal resolution (Bastiaens and Squire, 1999; Elangovan et al., 2002). 


\section{Chapter 2: MATERIALS}

\subsection{Chemicals}

PBS-Tabletten

Sigma

MTT Formazan

Sigma

Chlorpromazine hydrochloride

Sigma

Fibroblast Growth Factor - Basic

Sigma

Nystatin

Sigma

Crystal violet

Sigma

Glycerol, ACS, 99,5\%

Sigma

Poly-L-Lysine Hydrobromide

Sigma

Glucose (45\%) Solution

Sigma

Poly-L-Ornithine hydrobromide

Sigma

horse serum

Sigma

PBS-Tabletten

Sigma

Glutaraldehyde, Grade I, 25\%

Sigma

Glutaraldehyde, Grade I, 8\%

Sigma

Gelatin-Lösung

Sigma

Poly-L-Lysine Hydrobromide

Sigma

Collagen TypeIV

Sigma

Poly-L-Ornithine hydrobromide

Sigma

Cholesterol, powder

Sigma

Sodium-cholesterylsulfate

Sigma

Gelatin, typeA, powder

Sigma

5(6)-Carboxy-X-Rhodamine

Sigma

Rhodamine 800

Sigma

Rhodamine 101

Sigma

BDNF

Sigma

Fibronectin (solid)

Sigma

Methyl-b-Cyclodextrin

Sigma 
PBS-Tabletten

Sigma

Valinomycin

Sigma

Poly-L-Lysine $\mathrm{HBr}$

Sigma

Jump-Start Taq DNA-Polymerase

Sigma

BSA (Albumin bovine serum)

Sigma

Uridine

Sigma

Poly-D-Lysine hydrobromide

Sigma

Trypsin Inhibitor from chicken egg white

Sigma

5-Fluoro-2'-deoxyuridine

Sigma

L-Cysteine

Sigma

Poly-L-Lysine Hydrobromide

Sigma

Taq DNA-Polymerase

Sigma

Emetine dihydrochloride hydrate

Sigma

Transferrin

Sigma

Cytosin arabinfuranoside

Sigma

Insulin-Bovine

Sigma

Poly-L-ornithine hydrobromide

Sigma

Interleucin-1b-from mouse

Sigma

Aphidicolin

Sigma

PBS-Tabletten

Sigma

Phalloidin-FITC

Sigma

DMSO

Sigma

Glutaraldehyde solution

Sigma

Aphidicolin

Sigma

Tau protein human

Sigma

Tubilin from bovine brain

Sigma

Laminin

Sigma

Nimodipine

Sigma

Galanthamine $\mathrm{HBr}$

Sigma

LB Agar Powder

Sigma

LPA-Na-salt

Sigma

Triton X-114

Sigma 
Guanosine-5'-diphosphat-Na-salt

Sigma

Poly-L-Lysine Hydrobromide

Sigma

LPA-Na-salt

Sigma

PBS-Tabletten

Sigma

Trypton

Sigma

Fibronectin, 0.1\% Solution

Sigma

Natriumfluorid

Sigma

Aluminiumchlorid

Sigma

Tetraethylorthosilicat

Sigma

Cholera Toxin B subunit, Biotin

Sigma

Heparin, wt $6000 \mathrm{~mol}$

Sigma

Recombinant Mouse Netrin-1

R\&D Systems

Recombinant Human Semaphorin 3A/Fc Ch

Triethoxyphenylsilane

R\&D Systems

4-Aminobenzamidine x $2 \mathrm{HCl}$

Fluka

LAURDAN

Fluka

Fluka

Kaliumiodid

Fluka

Guanosine-5' -g.triphosphat-Na-salt

Fluka

Ammoniaklösung (28\%)

Fluka

Biotin

Fluka

Natriumhydroxid, p.A.

Roth

Methanol, gradient grade HPLC

Roth

Ethanol, vergällt

Roth

Iso-Propanol

Roth

Ethanol, p.A.

Roth

Borsäure, p.A.

Roth

Tris $\mathrm{HCl}$, p.A.

Roth

Wasser, steril

Roth

Ethanol, vergällt

Roth

Natriumhydroxid, p.A.

Roth

Methanol

Roth

Methanol, gradient grade HPLC

Roth 
Ethanol, vergällt

Roth

Tissue-Box (30 Stk.)

Roth

Vinyl-Gloves, Gr.S

Roth

Vinyl-Gloves, Gr.M

Roth

Vinyl-Gloves, Gr.L

Roth

Nitril-Gloves, Gr. S

Roth

Nitril-Gloves, Gr. M

Roth

Nitril-Gloves, Gr. L

Roth

Wischtüscher T (28 Rollen)

Roth

Essigsäure, p.A.

Roth

Titriplex III, EDTA

Roth

Borsäure

Roth

Ethanol, p.A.

Roth

Ethanol, vergällt

Roth

Wasser, steril

Roth

IPTG

Roth

Hefeextrakt

Roth

MES pufferan

Roth

EGTA, p.A.

Roth

Ethanol, vergällt

Roth

Methanol, p.A.

Roth

Rotipuran Aceton, p.A., ASC

Roth

Precision Plus Protein Marker

Bio-Rad

Empty Econo-Pac Columns

Bio-Rad

MiniProtean3-short plates

Bio-Rad

Precision Plus Protein (all blue standards)

Bio-Rad

Protein Assay Dye Reagent

Bio-Rad

\subsection{Cell culture medium, inhibitors and chemicals}

DMEM

Invitrogen

RPMI1640

Invitrogen 
Ionomycin

Calbiochem

b-Nerve growth factor, human

Calbiochem

ML-7 Hydrochlorid

Calbiochem

Colchicine

Calbiochem

Cytochalasine D

Calbiochem

Jasplakinolide

Calbiochem

Latrunculin A

Calbiochem

Micotubule-Interfering Agents Set

Calbiochem

Lactacystin

Calbiochem

Roscovitine

Calbiochem

Pepstatin A

Calbiochem

Leupeptin hemisulfate

Calbiochem

LnLL

Calbiochem

Chymostatin

Calbiochem

E-64L

Calbiochem

Heat shock protein70 inhibitor

Calbiochem

Heat shock protein 27 inhibitor

Calbiochem

Bcl-xL BH44-24 (human)

Merck Biosciences

Calpastatin Peptide

Merck Biosciences

Caspase Inhibitor I

Merck Biosciences

Caspase Inhibitor, neg.control

Merck Biosciences

\subsection{Molecular biology chemicals, competent cells and vectors}

Stbl2 comp. Cells

One Shot GeneHogs Comp cells

One Shot Top 10 Comp cells

One Shot BL21(DE3) Comp cells

p-EYFPn1 vector

p-EGFPn1 vector

p-ECFPn1 vector
Invitrogen

Invitrogen

Invitrogen

Invitrogen

BD Biosciences

BD Biosciences

BD Biosciences 
p-EGFPC1 vector

p-EGFPC3 vector

pd2EGFn1 vector

pd2ECFPn1 vector

MinElute Reaction CleanUp Kit

MinElute Gel Extraction Kit

QiaQuick PCR Purification Kit

Collection tubes, $2 \mathrm{ml}$

N-Terminus pQE-Vector-Set

MinEltue Gel Extraction Kit

QiaPrep Spin MiniPrep Kit

TransMessenger Transfection Reagent

Rneasy Mini Kit

Omniscript RT Kit

Rnase-Free Dnase Set
BD Biosciences

BD Biosciences

BD Biosciences

BD Biosciences

Qiagen

Qiagen

Qiagen

Qiagen

Qiagen

Qiagen

Qiagen

Qiagen

Qiagen

Qiagen

Qiagen

\subsection{Enzymes}

Not I

EcoR I

Dpn I

Sac II

Vent(exo-)-DNA-Polymerase

Bst-DNA-Polymerase

Klenow fragment 3' to 5' exo-

Streptavidin

Hind III

BamH I

Kpn I

DeepVent-DNA-Polymerase

Xho I

Xba I
New England Biolabs

New England Biolabs

New England Biolabs

New England Biolabs

New England Biolabs

New England Biolabs

New England Biolabs

New England Biolabs

New England Biolabs

New England Biolabs

New England Biolabs

New England Biolabs

New England Biolabs

New England Biolabs 
Sac I

Apa I

Sma I

Nhe I

Vent-DNA-Polymerase

Nco I

Pst I

Sal I

Sac II

Nde I

Taq-DNA-Polymerase

T4-Polynucleotide Kinsase (PNK)

Sma I

Vent-Polymerase

BspE I

Nru I

GM 2163, Dam Neg. Stamm

T4-DNA-Polymerase

Age I

T4-Polynucleotide kinase

BsiW I

Pml I

Pst I

Afe I

BsmB I

BsrG I

Pme I

Sac I

BsrG I

Taq-DNA-polymerase

T4-DNA-Ligase
New England Biolabs

New England Biolabs

New England Biolabs

New England Biolabs

New England Biolabs

New England Biolabs

New England Biolabs

New England Biolabs

New England Biolabs

New England Biolabs

New England Biolabs

New England Biolabs

New England Biolabs

New England Biolabs

New England Biolabs

New England Biolabs

New England Biolabs

New England Biolabs

New England Biolabs

New England Biolabs

New England Biolabs

New England Biolabs

New England Biolabs

New England Biolabs

New England Biolabs

New England Biolabs

New England Biolabs

New England Biolabs

New England Biolabs

New England Biolabs

Roche Diagnostics 
Alcaline Phosphatase, calv

SspB I

Nhe I

Bgl II

Ksp I

PinA I

FuGene 6 Transfection Reagent

Klenow Enzyme

Caspase-3 (CPP32)

Jump-Start Taq DNA-Polymerase
Roche Diagnostics

Roche Diagnostics

Roche Diagnostics

Roche Diagnostics

Roche Diagnostics

Roche Diagnostics

Roche Diagnostics

Roche Diagnostics

BD Biosciences

Sigma

\subsection{Antibodies}

Anti-Ubiquitin bovine (rabbit)

Anti-Proteosome 19S-Subunit S2 hm (rabbit)

Anti-tau AB, phosphos pecific (Ser262)

Anti-tau-phospho-specific (Ser422), Hman(rabbit)

Glia Fibrillary Acidic Protein

Sek. AB, goat-anti-mouse, cy3 conjugated

IgG (ab')-2-Fragment (Kaninchen, Maus)

Poly AB Goat-anti-mouse, cy5

Poly AB Goat-anti-rabbit-FITC

Poly AB Goat-anti-rabbit

Poly AB Goat-anti-mouse

IgG F(ab)2-fragment, Goat anti Rabbit - Cy5

IgG F(ab')2-fragment, Ziege, Maus, poly AB - FITC

Mouse X TAU (CT) Antibody

Mouse-Tau-AB-c-treminal (T46)

Monoclonal Anti-a-tubulin clone B-5-1-2

Monoclonal Anti-a-tubulin clone DM 1A
Calbiochem

Calbiochem

Calbiochem

Calbiochem

DAKO

Dianova

Dianova

Dianova

Dianova

Dianova

Dianova

Dianova

Dianova

Zytomed

Zytomed

Sigma

Sigma 
Monoclonal Anti-beta-tubulin III

Anti-Ezrin monoclonal AB clone 3C12

Anti-HA-tag, polyclonal AB

Antikörper monoclonal, HA tagged .11

Anti GFP antibody

Anti HSP70 antibody

Anti HSP27 antibody
Sigma

Sigma

MoBiTec

HISS Diagnostics

Roche diagnostics

Chemicon international

Chemicon international 


\section{Chapter 3: METHODS}

\subsection{MOLECULAR BIOLOGY PROTOCOL}

\subsubsection{Buffers and solutions}

3.1.1.1. Luria Bertani medium (LB)

10 g Bacto-trypton

$5 \mathrm{~g}$ bacto-yeast extract

$5 \mathrm{~g} \mathrm{Nacl}$

\subsubsection{LB ampicillin agar plates}

$1.5 \%$ agar in LB medium

$100 \mathrm{ug} / \mathrm{ml}$ of ampicillin

Poured at $55^{\circ} \mathrm{c}$ to $10 \mathrm{~cm}$ Petri plates

3.1.1.3. Transformation buffer 1 (TFB1) $30 \mathrm{mM}$ calcium acetate, ph 5.8

$100 \mathrm{mM}$ rubidium chloride

$10 \mathrm{mM}$ calcium chloride

$50 \mathrm{Mm}$ manganese chloride

$15 \%$ glycerol (w/v)

3.1.1.4. Transformation buffer 2 (TFB2) $10 \mathrm{~mm}$ MOPS, ph 6.5

$75 \mathrm{~mm}$ calcium chloride

$10 \mathrm{~mm}$ rubidium chloride

$15 \%$ glycerol $(\mathrm{w} / \mathrm{v})$

\subsubsection{Glycerol stock buffers}

$10 \% \mathrm{v} / \mathrm{v}$ of glycerol in water

$100 \%$ glycerol for frozen stock 
3.1.1.6. SOC medium

3.1.1.7. Buffer p1

3.1.1.8. Buffer p2

3.1.1.9. Buffer p3

\subsubsection{Restriction digestion Buffers}

3.1.2.1. NEB1

3.1.2.2. NEB2

3.1.2.3. NEB3
$0.5 \%$ yeast extract

$2 \%$ bacto-tryptone

$10 \mathrm{~mm}$ sodium chloride

$2.5 \mathrm{~mm}$ potassium chloride

$10 \mathrm{~mm}$ magnesium sulphate

$10 \mathrm{~mm}$ magnesium chloride

$20 \mathrm{~mm}$ glucose

$50 \mathrm{~mm}$ Tris/Hcl ph 8.0

$10 \mathrm{~mm}$ EDTA

100 ug/ml RNase A

$0.2 \mathrm{M} \mathrm{NaOH}$

$1 \% \operatorname{SDS}$

$3 \mathrm{M}$ potassium acetate ph 5.5

$10 \mathrm{~mm}$ Bis tris propane-hcl ph 7.0,

$10 \mathrm{~mm} \mathrm{mgcl} 2,1 \mathrm{~mm}$ DTT

$10 \mathrm{~mm}$ tris-hcl ph 7.9,

$10 \mathrm{~mm}$ mgcl2, $50 \mathrm{~mm}$ Nacl, $1 \mathrm{~mm}$ DTT

50 mm Tris-hcl ph 7.9,

10 mm mgcl2, 100 mm Nacl, 1 mm DTT 
3.1.2.4. NEB4

3.1.3. Gel loading buffer

3.1.4. PCR buffers

3.1.5. Cloning buffers
$20 \mathrm{~mm}$ Tris-acetate ph7.9,

$10 \mathrm{~mm}$ magnesium acetate,

$1 \mathrm{~mm}$ DTT, $50 \mathrm{~mm}$ potassium acetate

$0.25 \% \mathrm{w} / \mathrm{v}$ bromophenol blue

$40 \%$ saccharose in $1 \mathrm{x}$ TAE

10 reaction buffer NEB thermopol

DNTP mix Roche

Deep VENT polymerase NEB

CIP 10x de-phosphorylation buffer (roche)

SAP 10x de- phosphorylation buffer (roche)

Klenow fragment enzyme (NEB)

CIP or SAP enzymes (Roche)

\subsubsection{Chemical competent cells preparation}

Stocks of competent bacteria were streaked on to a LB agar plates and kept at $37^{\circ} \mathrm{C}$ overnight and a single colony is picked for inoculating in $5 \mathrm{ml}$ of starter culture, unless and otherwise mentioned and cultivated for 12 hours or more. $1 \mathrm{ml}$ of starter culture was inoculated into $99 \mathrm{ml}$ of pre warmed LB medium without any antibiotic, until and otherwise mentioned by providers of competent bacteria with internal resistance gene. Cultures were grown at $37^{\circ} \mathrm{c}$ until it reaches OD 0.6.cells were pellet down at 5000X g for $15 \mathrm{~min}$ at $4^{\circ} \mathrm{C}$, the bacterial pellet was re suspended into $0.2 \mathrm{ml}$ culture volume of buffer TFB1 and incubated on ice for $5 \mathrm{~min}$. Cells were pellet down at 3000X $\mathrm{g}$ for 15 min at $4{ }^{\circ} \mathrm{C}$ and re-suspended in $0.04 \mathrm{ml}$ culture volume of buffer TFB2 and incubated for $30 \mathrm{~min}$ on ice before aliquot them as $50 \mathrm{ul}$ fractions. The cells were quick frozen at $-80^{\circ} \mathrm{C}$. 


\subsubsection{Preparation of electro-competent cells}

Stocks of competent bacteria were streaked on to a LB agar plates without antibiotic until and otherwise mentioned and were kept at $37^{\circ} \mathrm{C}$ overnight. Single colony from bacteria grown overnight was picked for inoculating in $5 \mathrm{ml}$ of starter culture and cultivated for 12 hours or more. $1 \mathrm{ml}$ of starter culture was inoculated into $99 \mathrm{ml}$ of pre warmed LB medium without any antibiotic until and otherwise mentioned by providers of competent bacteria with internal resistance gene. Cultures were grown at $37^{\circ} \mathrm{C}$ until it reaches Optical Density (OD) of 0.6. The cultured cells were pre chilled on ice for $15 \mathrm{~min}$ and pellet down at $5000 \mathrm{Xg}$ for $15 \mathrm{~min}$ at $4^{\circ} \mathrm{C}$. Pellet were resusupended in $500 \mathrm{ml}$ of sterile ice cold water and centrifuged for $15 \mathrm{~min}$ at $5000 \mathrm{Xg}$, repeated for one more time. To the pellet, desired volume of sterile distilled water is added to resuspend and aliquot into $100 \mathrm{ul}$ stocks. The stocks were quick-frozen with liquid nitrogen and were stored at $-80^{\circ} \mathrm{c}$.

\subsubsection{Determining the concentration of DNA}

DNA concentration was determined using a spectrophotometer by its principle of absorbing DNA at $260 \mathrm{~nm}$. DNA was diluted in water at appropriate dilution and was measured for the absorption at $260 \mathrm{~nm}$. Absorbance or OD of 1 at $260 \mathrm{~nm}$ corresponds to approximately $50 \mathrm{ug} / \mathrm{ml}$ for double stranded DNA or $40 \mathrm{ug} / \mathrm{ml}$ for single stranded DNA and RNA or $20 \mathrm{ug} / \mathrm{ml}$ of oligo-nucleotides. The ratio between the OD at 260 and 280 was counted to calculate the relative purity of nucleic acids. Pure sample will be of the OD 1.8 and 2.0 for DNA and RNA respectively and gives a measure for relative contamination.

\subsubsection{Transformation of DNA to chemical competent cells}

The sample DNA of 50 -100 ng of DNA was mixed with $50 \mathrm{ul}$ of competent bacterial aliquot and incubated on ice for $30 \mathrm{~min}$. Cells were subjected to heat-shock in a water bath at $42^{\circ} \mathrm{C}$ for 45 seconds and incubated on ice for $5 \mathrm{~min}$. To the heat-shock treated transformation mixture, $1 \mathrm{ml}$ of LB medium was added and incubated at $37^{\circ} \mathrm{C}$ for 1 hour. 
The transformed samples of desired volume was plated on LB-plates with appropriate antibiotic.

\subsubsection{Transformation of DNA to Electro competent cells}

$50 \mathrm{ng}$ of DNA was added to an aliquot of $50 \mathrm{ul}$ electro-competent cells, incubated on ice for $5 \mathrm{~min}$ and the cells were transferred into disposable electroporation cuvette. The electroporation mixture was electroporated by applying 2 times 2000 watt and the cells were mixed with $1 \mathrm{ml}$ of SOC medium, incubated for 1 hour at $37^{\circ} \mathrm{c}$ and the desired volume of cells were plated onto a LB agar plate with appropriate antibiotic.

\subsubsection{Preparation of Glycerol stock}

Bacteria colonies harboring plasmids was grown in LB medium with respective antibiotic at $37^{\circ} \mathrm{c}$ overnight. From the cultures grown overnight desired amount of amount of stock were frozen at a concentration mixture of $0.3 \mathrm{ml}$ of bacterial culture and $0.7 \mathrm{ml}$ of $100 \%$ glycerol in a cryo-preservation tubes. The cells were quick frozen by immersing them using liquid nitrogen and stored at $-80^{\circ} \mathrm{c}$.

\subsubsection{Agarose gel}

The size and purity of DNA was analyzed using agarose gel electrophoresis, DNA of multiple size can be resolved using agarose gels, based on their molecular size, shape and net charge under the influence electrical field. The DNA can only be resolved accordingly to their relative size and the gel was made according to the size of DNA to be resolved. Molecular size of DNA ranges from few 100 bases to $20 \mathrm{~Kb}$ can be resolved in differential proportion of gel mixture, lower the DNA size higher will be the percentage of gel mixture. 


\begin{tabular}{|l|l|}
\hline Agarose concentration $(\%)$ & DNA size $(\mathrm{kb})$ \\
\hline 0.5 & $20-1.5$ \\
\hline 0.7 & $12-1.0$ \\
\hline 1.0 & $7-0.5$ \\
\hline 1.2 & $4-0.4$ \\
\hline 1.5 & $3-0.2$ \\
\hline 2.0 & $2-0.1$ \\
\hline
\end{tabular}

\subsubsection{Mini preparation of plasmid DNA}

Plasmid DNA was purified using ready-made kit from qiagen, following the manufacturers instruction. Single colony of desired bacteria was grown in a $5 \mathrm{ml}$ of LB medium with appropriate antibiotic at $37^{\circ} \mathrm{c}$ overnight. The cultured bacterial cells were pelleted at 5000X g for $5 \mathrm{~min}$, resuspended in $0.25 \mathrm{ml}$ of resuspension buffer P1, lysed with $0.25 \mathrm{ml}$ of lysis buffer P2 and neutralized with $0.35 \mathrm{ml}$ of neutralization buffer N3. The cells after lysis were mixed thoroughly and centrifuged for $15 \mathrm{~min}$ at $12,000 \mathrm{Xg}$. The supernatant from the centrifuged fractions was loaded into spin column (qia mini prep) and spun for $1 \mathrm{~min}$ at $12,000 \mathrm{Xg}$ and the DNA was bound to column using $0.5 \mathrm{ml}$ of binding buffer $\mathrm{PB}$ and centrifuged at $12000 \mathrm{Xg}$ for $1 \mathrm{~min}$ and washed with $0.75 \mathrm{ml}$ of wash buffer PE and centrifuged at 12,000X g for one min. previous step was repeated again to remove the residual PE buffer. The spin column was air dried at RT for 2 min and the DNA was eluted using water of 50 to $100 \mathrm{ul}$ of water or elution buffer in an eppendorf tubes depends on the need and storage.

\subsubsection{Restriction Digestion DNA}

Restriction enzymes are used in terms of measuring unit as number of 'Units' (U) 
One unit of restriction enzymes is the amount of enzyme required to digest $1 \mathrm{ug}$ of substrate DNA in 1 hour

\author{
Plasmid DNA $\quad 0.5-3.0$ ug \\ Reaction buffer (10X) 2 ul \\ Restriction enzyme $\quad 1-5$ unit \\ BSA (if needed) $\quad 1 \mathrm{ug} / \mathrm{ul}$ \\ Water to make up to $20 \mathrm{ul}$ of reaction volume
}

Reaction mixture was incubated for $1-2$ hour at $37^{\circ} \mathrm{C}$ and temperature was adopted or changed accordingly, from the user instruction manual from the enzyme providers. The restriction digestion was analyzed by loading the restricted samples on an agarose gel ranging from 05-2 percent depending on the size of desired plasmid and insert released.

\title{
3.1.15. Polymerase chain reaction (PCR)
}

Template (plasmid, cDNA) $\quad 50 \mathrm{ng}$

Reaction buffer (10x) $\quad 5.0 \mathrm{ul}$

DNTPs mixture (100 um) $\quad 1.5 \mathrm{ul}$

Primer FW (100 pm) $\quad 1.25 \mathrm{ul}$

Primer RV $(100 \mathrm{pm}) \quad 1.25 \mathrm{ul}$

Enzyme Deep VENT polymerase $1.0 \mathrm{ul}$

Water made up to 50ul total reaction volume

\subsubsection{Temperature profile}

Initial denaturation $\quad 95^{\circ} \mathrm{c}$ for $5 \mathrm{~min}$

Final denaturation $\quad 95^{\circ} \mathrm{c}$ for 45 seconds

Annealing $55-60^{\circ} \mathrm{c}$ for 45 seconds 
Initial extension

Final extension $72^{\circ} \mathrm{c}$ for $1 \mathrm{~min} / \mathrm{kb}$ DNA size

$72^{\circ} \mathrm{c}$ for $5 \mathrm{~min}$

Reaction mixture was analyzed in agarose gel for the degree of amplification. The control samples without enzyme or plasmid were used to check the product amplified, possible cross reactivity and amplification error by buffer contamination.

\section{Temperature Profile}

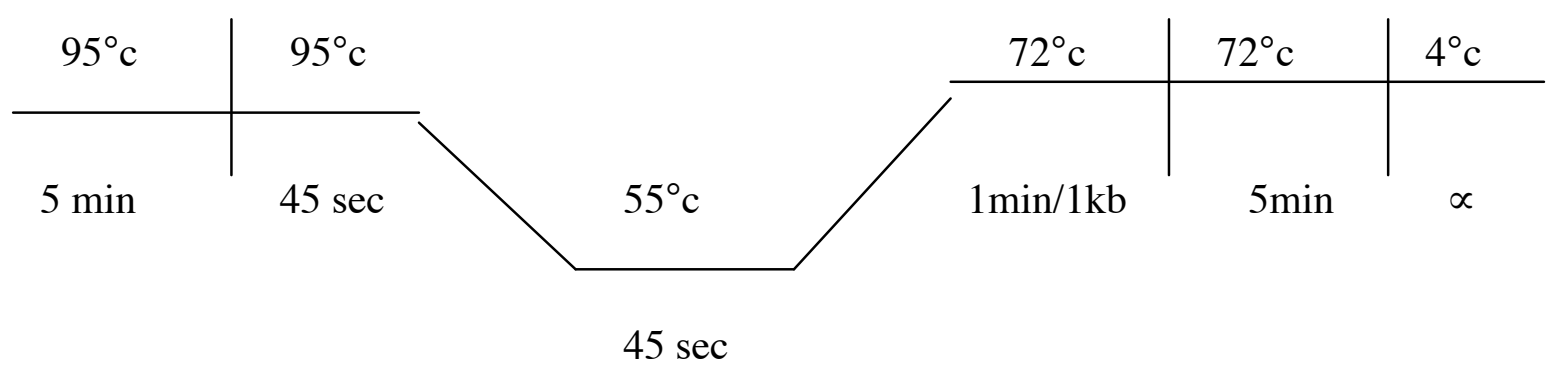

\subsubsection{Random Mutagenesis of EYFP}

Plasmid DNA consist of YFP gene, approximately about $100 \mathrm{ng}$ was transformed into XL1RED strain of E.coli bacteria, which was known for containing mutant DNA polymerase that lacks 3' to 5' exo-nuclease proof reading capacity. Since the mutant DNA polymerase in XL1RED competent bacteria were incapable of proof reading the DNA amplified, random mismatches were introduced in non-specific regions of DNA. The transformed samples were incubated 1 hour at $37^{\circ} \mathrm{C}$ before plating them into a $60 \mathrm{~cm}$ Petri dishes plates and kept overnight at $37^{\circ} \mathrm{C}$. The bacterial colonies from whole plates were scraped and dissolved in $100 \mathrm{ml}$ of LB medium, which was further resuspended and centrifuged at $5000 \mathrm{X}$ g for $15 \mathrm{~min}$. The pelleted bacteria's were used for isolating DNA by following the standard qiagen ready kit protocol. From the DNA made, 10ng of it was then retransformed into a BL21DE3 strain of E.coli bacteria, which was then plated onto a LB medium with appropriate antibiotic and $0.2 \mathrm{~mm}$ of IPTG $/ \mathrm{ml}$ of LB agar. The 
colonies obtained was then subjected to manual inspection for their mutant phenotype, since it was a fluorescent protein in our case, we inspect the colonies with UVillumination for the change in chromophoric phenotype

\subsubsection{Site directed mutagenesis}

Site directed mutagenesis is the alternative method to specifically mutate the DNA or plasmid of interest at a desired nucleotide level. For this the PCR primers were designed that can replace the desired nucleotide from the original nucleotide of the template DNA by PCR amplification.

Template

Reaction buffer (10x)

DNTPs mixture (100 um)

Primer FW (100 pm)

Primer RV (100 pm)

Enzyme Deep VENT polymerase

Water 50-100ng

5.0ul

$1.5 \mathrm{ul}$

$1.25 \mathrm{ul}$

$1.25 \mathrm{ul}$

1.0ul (added after initial denaturation) made up to $50 \mathrm{ul}$ total reaction volume

\subsubsection{Temperature profile}

Initial denaturation

Final denaturation

Annealing

Initial extension

Final extension $95^{\circ} \mathrm{C}$ for $5 \mathrm{~min}$

$95^{\circ} \mathrm{C}$ for 45 seconds

$55-60^{\circ} \mathrm{C}$ for 45 seconds

$72^{\circ} \mathrm{C}$ for $1 \mathrm{~min} / \mathrm{kb}$ DNA size

$72^{\circ} \mathrm{C}$ for $5 \mathrm{~min}$

The product of the PCR amplification was confirmed with a control samples without enzyme and cleaned with qiagen PCR purification kit. Bacterial DNA (original template) was removed from the PCR amplified product, by digesting the samples with enzyme 
Dpn1. The enzyme Dpn1 selectively digest the bacterial DNA bearing methylated bases at the beginning of template.

\title{
3.1.18. Dpn1 digestion
}

PCR sample template

10X buffer

Enzyme Dpn1

Water
2-4 ug of DNA

$2 \mathrm{ul}$

$1-2 \mathrm{ul}$

made up to 20ul reaction volume

Reaction samples were incubated at $37^{\circ} \mathrm{C}$ for $1-2$ hours and checked for template digestion in gel. (If required) From the $20 \mathrm{ul}$ of reaction mixture $5 \mathrm{ul}$ of amplified product was then transformed into BL21DE3 E.coli bacterial strain. (See transformation protocol). The colonies were picked for plasmid preparation and the DNA was confirmed by sequencing for desired mutation.

\subsubsection{Molecular Cloning and Vector construction}

\author{
DNA for Vector backbone 1-3ug \\ DNA for Insert 2-3ug
}

DNA for vector backbone and insert are digested with respective restriction endonuclease and checked for appropriate vector and insert size in an agarose gel (See agarose gel protocol) and further ligated. 


\subsubsection{Ligation}

Gel extracted vector backbone and insert are mixed in a ratio of 1:4 for Vector: Insert, and further ligated using enzyme T4 DNA ligase
Vector
50-200ng
Insert
$150-600 \mathrm{ng}$
Ligase buffer (10x)
2ul
T4 DNA ligase
$1 \mathrm{ul}$
Water
to make the reaction mixture to $20 \mathrm{ul}$

Kept at RT or $16^{\circ} \mathrm{C}$ for 2 hours and 2-5ul of ligated DNA was transformed into an XL1 Blue E.coli bacterial strain for plasmid preparation and DNA analysis.

\subsection{BIOCHEMICAL METHODS}

\subsubsection{Buffers and solutions}

\subsubsection{Wash buffer for bacterial cell pellet $\mathrm{Ph} 7.5$}

Tris-Base

$\mathrm{Nacl}$
$50 \mathrm{~mm}$

$300 \mathrm{~mm}$

\subsubsection{Lysis buffer for protein extraction}

Tris-Base

Triton X-100

Or lysozyme
$50 \mathrm{~mm}$

$1 \%$

1ug/ml 


\subsubsection{Equilibration buffer pH 8.0 (talon)}

Tris-Base

$\mathrm{Nacl}$

3.2.1.4. Wash buffer pH 7.0 (talon)

Tris-Base

$\mathrm{Nacl}$

Imidazole

3.2.1.5. Elution buffer pH 7.0 (talon)

Tris-Base

$\mathrm{Nacl}$

Imidazole
$50 \mathrm{~mm}$

$300 \mathrm{~mm}$
$50 \mathrm{~mm}$

$300 \mathrm{~mm}$

$5 \mathrm{~mm}$

\subsubsection{Dialysis buffer (PBS or TRIS ph 8.0)}

Phosphate buffered saline ( $\mathrm{Ph} 8.0)$

$50 \mathrm{~mm}$

$300 \mathrm{~mm}$

$300 \mathrm{~mm}$

$\mathrm{KH} 2 \mathrm{PO} 4$

K2HP04

$\mathrm{Nacl}$

0.1M TRIS base

$0.3 \mathrm{M} \mathrm{Nacl}$

\subsubsection{Strip buffer}

Tris-Base

Nacl

EDTA
$20 \mathrm{~mm}$

$300 \mathrm{~mm}$

$100 \mathrm{~mm}$ 
3.2.1.8. Charge buffer

3.2.1.9. Sample buffer

(Laemmli buffer 2X)

3.2.1.10. Stacking gel (buffer)

3.2.1.11. Separating gel buffer

3.2.1.12. Ammonium per-sulfate (APS)
$50 \mathrm{mM} \mathrm{NisO}_{4}$ in 1 liter of water

125mm TRIS-Hcl, pH 6.8

$4 \%$ SDS

0.004\% Bromophenol Blue

$20 \%(w / v)$ Glycerol

$20 \mathrm{~mm}$ DTT

1.0M Tris-Hcl Ph 6.8

1.5M Tris-Hcl pH 8.8

$30 \%$ Acrylamide,

29.2g acryl amide, Bis-Acrylamide mixture

$0.8 \mathrm{~g}$ bis-acrylamide, $100 \mathrm{ml}$ of water

$10 \%(\mathrm{w} / \mathrm{v})$

3.2.1.13. Sodium Do-decyl Sulphate (SDS) $10 \%$ w/v

3.2.1.14. 10x Running buffer: 1 L

3.2.1.15. 10x Blotting buffer: 1 L

1x Blotting buffer: $2 \mathrm{~L}$
$30.3 \mathrm{~g}$ Tris base $(=0.25 \mathrm{M})$

$144 \mathrm{~g}$ Glycine $(=1.92 \mathrm{M})$

$10 \mathrm{~g} \operatorname{SDS}(=1 \%)$--add last

$30.3 \mathrm{~g}$ Tris base $(=0.25 \mathrm{M})$

$144 \mathrm{~g}$ Glycine (=1.92 M), pH 8.3

To make $2 \mathrm{~L}$ of $1 \mathrm{x}$ Blotting buffer:

$400 \mathrm{ml}$ Methanol

$200 \mathrm{ml}$ 10x Blotting buffer

$1400 \mathrm{ml}$ with water 
3.2.1.16. Blocking buffer

3.2.1.17. PBST

3.2.1.18. Stripping buffer $0.5 \mathrm{~L}$
$5 \mathrm{~g}$ skimmed milk powder (cholesterol free)

$100 \mathrm{ml}$ of PBS

phosphate buffered saline

$0.1 \%$ Tween $(\mathrm{v} / \mathrm{v})$

$0.2 \mathrm{M}$ Glycine, $\mathrm{pH} 2.5$

$0.05 \%$ Tween 20

(Sterile filter solution at $4^{\circ} \mathrm{C}$ )

$50 \%(\mathrm{v} / \mathrm{v})$ Methanol

$10 \%(\mathrm{v} / \mathrm{v})$ Acetic acid

$50 \%(\mathrm{v} / \mathrm{v})$ water

$0.5 \%$ Coomassie brilliant blue

\subsubsection{Gel de-staining solution $50 \%(\mathrm{v} / \mathrm{v})$ Methanol \\ $10 \%(\mathrm{v} / \mathrm{v})$ Acetic acid \\ $50 \%(\mathrm{v} / \mathrm{v})$ water}

\subsubsection{Protein expression and purification}

Respective wild type and mutant plasmids in bacterial expression vectors PRSETB 6x HIS tag was transformed into a BL21DE3 E. coli bacterial expression system. Single colony was picked and inoculated into a starter culture of $25 \mathrm{ml} \mathrm{LB}$ amp and were grown at 37 degree overnight. Mass culture of $500 \mathrm{ml} \mathrm{LB}$ amp was made by inoculating $1 \% \mathrm{v} / \mathrm{v}$ of starter culture. The cultures were kept at $37^{\circ} \mathrm{C}$ and Optical Density (OD) of the culture medium was measured every hour until it was 0.6 OD. The bacterial cultures at 0.6 OD were induced with $0.4 \mathrm{mM}$ IPTG (constitutive inducer for lactose promotor) for 2 hour at $37{ }^{\circ} \mathrm{C}$. The induced cultures were ethanol shocked by mixing it with $2 \% \mathrm{v} / \mathrm{v}$ ethanol premixed in $500 \mathrm{ml}$ of LB amp and transferred into incubation temperature of $20^{\circ} \mathrm{C}$ for 5 
hours to initiate slow expression and better folding. Further the cells were harvested by pelleting them at $5000 \mathrm{X} \mathrm{g}$ for $20 \mathrm{~min}$. The sample was then processed for protein extraction using Bug buster lysis kit as follows.

Bacterial pellet was resuspended in a bug buster lysis solution at a concentration of 1 gram of cells $/ 5 \mathrm{ml}$ of solution and were incubated for $30 \mathrm{~min}$ at RT. The lysed samples were centrifuged at $12000 \mathrm{Xg}$ for $45 \mathrm{~min}$ and the supernatant of it was mixed with metal affinity (Cobalt) talon resins. The resins were pre- equilibrated with equilibration buffers, which allows the binding of protein to column. The samples bound to bead were loaded into Econopack-column (Bio-Rad) and allowed to flow by gravity flow. Column was washed with twice the column volume using wash buffer and eluted in a desired volume using elution buffer. Eluted samples were further analyzed for the characteristic protein either by SDS-PAGE gel or by western blot analysis (is discussed in the later section of methods). For long term use proteins were dialysed (in appropriate buffer), concentrated (to desired volume) and frozen for cryo preservation

\subsubsection{Protein quantification using BIORAD reagent}

Standard BSA stock of $1 \mathrm{mg} / \mathrm{ml}$

Working standard range 2-20ug

Standard BSA curve was made using the concentration range of 2-20ug in a series of tubes and unknown protein concentration were measured by using 10ul of concentrated unknown protein sample, The diluted series of standard and unknown protein sample were made up to $0.8 \mathrm{ml}$ with water and $0.2 \mathrm{ml}$ of BIORAD reagent was mixed and incubated for $5 \mathrm{~min}$ at RT, $0.2 \mathrm{ml}$ of each sample was used to measured at $595 \mathrm{~nm}$ in the ELISA reader. 


\subsubsection{Spectro-photometry}

Protein sample of equal concentration were diluted in Tris buffer ph 8.0 and measured for their absorption characteristic in a spectrophotometer with a absorption range of 200$600 \mathrm{~nm}$

\subsubsection{SDS-PAGE (Poly Acrylamide Gel Electrophoresis)}

\begin{tabular}{|l|l|l|l|l|l|l|}
\hline Comp/\% Gel For 10ml & 6 & 8 & 10 & 12 & 15 & Stacking For 5ml \\
\hline 30\% Acryl amide & 2.0 & 2.7 & 3.3 & 4.0 & 5.0 & 0.83 \\
\hline Separating Gel Buffer & 2.5 & 2.5 & 2.5 & 2.5 & 2.5 & 0.63 \\
\hline Water & 5.3 & 4.6 & 4.0 & 3.3 & 2.3 & 3.4 \\
\hline Temed & 0.008 & 0.008 & 0.008 & 0.008 & 0.008 & 0.008 \\
\hline $10 \%$ APS & 0.3 & 0.3 & 0.3 & 0.3 & 0.3 & 0.15 \\
\hline
\end{tabular}

SDS PAGE is a biochemical technique preferably used to analyse the quality and quantity protein of interest based on the principle of charge to mass ratio, when the protein where charged with the anionic detergent SDS and resolved using a gel matrix of different porosity they get separated by their difference in the molecular size and migrates towards the anode with a unique charge. Two glass plates of preferred thickness were sealed by a spacer or adapter were used to pour a gel of different composition, initially separating gel were poured and left for 15 min until the gel gets polymerized and the upper layer was covered with iso-propanol to get a uniform layer and leave it undried. Iso-propanol was discarded and washed with water to remove the trace of alcohol. Stacking gel was poured over the separating gel and covered until the top gel plate to 
place a comb (10-15wells) with desired volume (40-25ul) and number of lanes without air bubble. After 30 min combs were removed and washed with water to remover the trace of unpolymerised gel. Samples boiled with sample buffer of desired volume were loaded and separated at a power range of 100-150 voltages depending on the size of the protein and percentage of polymerised gel.

\subsubsection{Spectro-fluorimetry}

Proteins of YFP mutants were diluted in TRIS Hcl buffer of ph 8.0 at equal concentration and was measured for their excitation and emission behavior, Spectrofluorimetric cuvette of $1 \mathrm{~nm}$ slit width is used for all the measurement (Studies were carried out in)

Excitation scan was obtained by keeping a constant emission of 540nm with excitation range of $400-535 \mathrm{~nm}$ and emission scan was obtained by keeping constant excitation of $490 \mathrm{~nm}$ with emission range of 495-600nm

\subsection{CELL CULTURE METHODS}

\subsubsection{Buffers and solutions}

3.3.1.1. CHO cells medium

BME

$10 \%$ FCS

$1 \% \mathrm{p} / \mathrm{s}(100 \mathrm{U} / \mathrm{ml})$

$1 \%$ Glutamine filter sterilized

\subsubsection{Neuroblastoma cells medium $\quad$ RPMI 1640 \\ $10 \%$ FCS \\ $1 \% \mathrm{p} / \mathrm{s}(100 \mathrm{U} / \mathrm{ml})$, \\ $1 \%$ Glutamine filter sterilized}


$1 \%$ neuronal serum

$2 \% \mathrm{~B} 27$ growth factor

$1 \%$ glucose filter sterilized

\subsubsection{Pc12 cells medium}

\subsubsection{Hela cells medium}

\subsubsection{MCF-7 cells medium}

\section{DMEM}

$10 \%$ FCS

$5 \%$ horse serum

$1 \% \mathrm{p} / \mathrm{s}(100 \mathrm{U} / \mathrm{ml})$

DMEM

$10 \%$ FCS

$1 \% \mathrm{p} / \mathrm{s}(100 \mathrm{U} / \mathrm{ml})$

$1 \%$ Glutamine filter sterilized

\section{DMEM}

$10 \%$ FCS

$1 \% \mathrm{p} / \mathrm{s} 100 \mathrm{U} / \mathrm{ml})$

$1 \%$ Glutamine filter sterilized

Cells were cultured in a tissue culture flask for about $70-90 \%$ density and split into second generation or propagation in a fresh culture flask, depends on the cell type $\mathrm{CHO}$, Hela, MCF-7, were split with trypsination and other types like neuroblastoma, pc12 were simply disrupted with mechanical force from pipette. Seeding in the cover slips will be used in a preferential density depends on the type of work, for live cell measurement and imaging about 20-30 percent before transfection and for western blotting, protein preparation about $60-80$ percent before transfection 


\subsubsection{Coating and seeding neurons for collapse assay}

The culture dishes were coated with PLO and laminin in the following order, first with PLO (poly-1-Ornithine) in PBS and let coat for overnight at $37^{\circ} \mathrm{c}$, washed with PBS before coating with laminin in PBS. Neurons used for collapse assay were seeded in a culture dishes coated only with PLO and no laminin.

\subsubsection{Fixing cell's with PFA or METHANOL}

Cells for immunocytochemistry or imaging were rinsed in PBS and fixed with 4\% PFA for $20 \mathrm{~min}$ at $\mathrm{RT}$ or $100 \%$ methanol for $5 \mathrm{~min}$ at $4^{\circ} \mathrm{c}$. Washed once with PBS and quenched using quenching buffer Tris Nacl pH 8.0 and mount using 5-10ul mowiol with or without antibleaching agent at RT for three hours.

\subsubsection{Transfection}

Cells seeded in culture dishes were transfected using the Effectene transfection reagent a ready kit from qiagen. Cover slips of diameter $25 \mathrm{~mm}$ were sterilized and placed in the bottom of desired culture dish. The culture dish were seeded with 30-50\% cells and grown at RT for 16 hours before transfection. DNA concentration of $0.6 u g$ were used for each well of 6 well plates and mixed with $4 \mathrm{ul}$ of enhancer reagent and incubated for 5 min at RT, spun for $1 \mathrm{sec}$ before adding 12.5ul of Effecteene reagent and allowed to form a complex at RT for $15 \mathrm{~min}$.

\subsubsection{PC12 cells differentiation and transfection}

PC12 cells were grown in DMEM medium supplemented with 5\% horse serum and 10\% goat serum with $100 \mathrm{ng} / \mathrm{ml}$ of NGF for differentiation. Culture dish or cover slips used to 
grow PC12 cells were pre coated with collagen $1 \mathrm{mg} / \mathrm{ml}$ stock to be diluted 1:100 before coating. Cells of $40 \%$ density are let grow for two to three days and medium is changed with fresh differentiation medium. The differentiation protocol was complete after 7 to 9 days and the cells were split by simple resuspension before coating again.

\subsubsection{Cryo-preservation of cells}

Cell lines of all kinds were stored for years in frozen aliquots. Cells of 70 to $90 \%$ densities were split or resuspended in a suitable buffer and centrifuged. The pellet can be stored into 3 to $4 \mathrm{ml}$ frozen aliquots with a medium supplemented with 10\% DMSO. The cells were cooled down slowly in $-70^{\circ} \mathrm{c}$ freezer before quick-freeze them in a liquid nitrogen cryo cans

\subsection{WESTERN BLOTTING}

Western blotting is a biochemical method commonly used to analyze the quality and quantity of protein. It is a precise and substrate specific method of analyzing protein. It works by the principle of affinity-labeling and antigen-antibody interaction. This method is applied to detect the protein or antigen, which are synthesized or expressed in time with a specificity of its interacting first antibody raised against the specific protein or antigen of interest. The antigen -antibody complex were detected by a double labeling of second antibody tagged with an enzyme which is species specific where in the first antibody was produced. The antigen -antibody complex tagged with a substrate specific enzyme was treated against the colored substrate to detect the protein of interest resolved by their size in a PAGE gel electrophoresis. 


\subsubsection{Preparation of cell lysate}

Medium in the culture dish were removed and the cells were washed twice with PBS, scraped in required volume and centrifuged at $12,000 \mathrm{Xg}$ for $5 \mathrm{~min}$ at $4^{\circ} \mathrm{c}$. Pellet was then resuspended in a lysis buffer or RIPA buffer and passed through a 21-gauge needle 3-4 times to shear the DNA and membrane. Partially lysed cells were incubated on ice for 30$60 \mathrm{~min}$ and spun at $15,000 \mathrm{Xg}$ for $20 \mathrm{~min}$ at $4^{\circ} \mathrm{c}$. Supernatant were removed and mixed with $4 \mathrm{X}$ loading buffer and 1ul of DTT or mercapto-ethanol. Samples were boiled for 10$20 \mathrm{~min}$ at $95^{\circ} \mathrm{c}$ in a heating block.

\subsubsection{Blot transfer}

Filter papers wattMann no-1, approximately 6 to the right size of gel and membrane to make a cassette. Nitrocellulose membrane and filter papers were soaked in transfer buffer for $15 \mathrm{~min}$ before start. Western cascade was made by placing 3 filter papers over the cathode (red), Membrane on top of it followed by gel and three more filter papers. The cassette is protected by western cushion on either side to provide an air tight sealing to membrane and gel. Western blot cassette was prepared in a following order as represented in the picture below

\section{+ Cathode}

\section{Pads}

Papers

\section{Membrane}

Gel

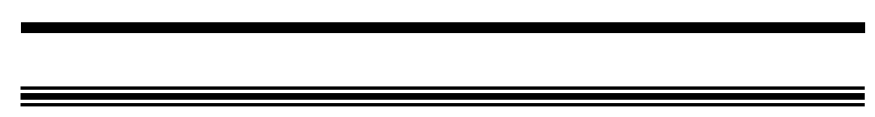

\section{Paper}

Pads

\section{- Anode}


Western cascade was inserted into a transfer block and placed in an appropriate orientation. Transfer of protein to membrane was carried out at a constant voltage ranging from 100-120 for 2 hours at $4^{\circ} \mathrm{c}$ with an ice block to avoid over heating of buffer and in turn the membrane. Membrane with protein was washed in PBS and blocked with 5\% BSA in PBS or 5\% skimmed milk powder (cholesterol free) for 1 hour at RT or $4^{\circ} \mathrm{c}$ overnight. Primary antibody of dilution ranging from 1:1000-3000 was added to the blocked membrane after washing it with PBST for 3 times 5 min each. Membrane was incubated at RT for 1 hour or overnight at $4^{\circ} \mathrm{c}$. Membrane was washed again with PBS three times for 5 min each before adding secondary antibody HRP conjugate at a dilution ranging from 1:5000-10,000 for 1 hour at RT or $4^{\circ} \mathrm{c}$ overnight. Treated membrane was washed again for three time $5 \mathrm{~min}$ each with PBST and visualized with a chemiluminescent substrate ECL- plus for 2 min after developing the bands in a photographic film by simple fixing and developing procedure.

\subsection{Imaging Techniques}

\subsubsection{Confocal and flourescence microscopy}

Imaging was done using the Leica SP-2 microscope with single-photon excitation in confocal mode. The microscope is equipped with an acousto-optical beam splitter (AOBS) for custom emission wavelength selection.

\begin{tabular}{|l|l|l|}
\hline Colours and Dyes & Excitation $(\mathrm{nm})$ & Emission Range (nm) \\
\hline CFP & 456 & $465-500$ \\
\hline GFP & 488 & $495-530$ \\
\hline YFP & 514 & $520-560$ \\
\hline CY3 & 563 & $570-630$ \\
\hline Cy5 & 633 & $640-750$ \\
\hline
\end{tabular}




\subsubsection{Immuno-cytochemistry or Immuno-fluorescence}

Cell culture medium was aspirated from the dish, washed with PBS once and fixed with 4\% PFA/0.25\% glutaraldehyde for 15-30 min at RT, washed again in PBS for $2 \mathrm{~min}$ and permeablised with $0.1 \%$ triton X-100 for $30-45$ min at RT. The permeabilisation mixture were aspirated and cells were washed with PBS two times at 2 min interval, blocked with 2\% BSA dissolved in PBS for 1-2 hours at RT. Cells were washed again with PBS for three times and incubated with primary antibody diluted in PBS of dilution ranging from 1:1000-3000 for 2-3 hours at RT or $4^{\circ} \mathrm{c}$ overnight. Further probed with secondary antibody tagged to fluorescent dyes, in a dilution ranging from 1:100-300 for 1-3 hours at RT or overnight at $4^{\circ} \mathrm{c}$. Extensive washing in PBS were required before mounting the samples in a mowiol with or without anti-bleaching reagent depending on the requirement of the samples and imaging technique.

\subsubsection{FqRET analysis}

Images of the GFP and Cy5 emission intensities were acquired using the Leica SP-2 confocal microscope with single-photon excitation in confocal mode. The microscope is equipped with an acousto-optical beam splitter (AOBS) for custom emission wavelength selection. GFP was excited using the $488 \mathrm{~nm}$ argon laser line; Cy5 was excited using the $633 \mathrm{~nm}$ HeNe laser line. Fluorescence emission was collected in spectral windows of 495-530 nm and 640-750 nm for GFP and Cy5, respectively. Ratios were calculated from the intensity division of GFP/CY5.

\subsubsection{Fluorescence Lifetime Microscopy: TD-FLIM}

The fluorescence decays were time-resolved by time-correlated single-photon counting (TCSPC) by the use of a SPC830 acquisition boards and SPCimage software (both from Becker\&Hickl GmbH, Berlin, Germany). Two-photon excitation of GFP was performed 
at $900 \mathrm{~nm}$ by a Ti:Sapphire Mira900F laser pumped by a Verdi-V8 laser (both from Coherent Inc.) and working in the femto-second mode-locked regime with a repetition rate of 76MHz. The laser beam was fed to the scanning head of a Leica TSC SP2 AOBS confocal microscope and the fluorescence light was collected by a multi channel plate photomultiplier (R3809U-50 by Hamamatsu Photonics) through a band-pass GFP filter $515 \pm 30 \mathrm{~nm}$ (EGFPHQset by AHF Analysentechnik AG) hosted on a custom-built filterwheel connected to the output optical port of the scanning-head. This allowed the filter to be removed to perform experiments on the complete emission spectral band. Analysis of the fluorescence transients was performed with the SPCimage software, which results were exported and analysed using an in-house developed Matlab toolbox. 


\section{Hypothetical model for local protein turnover: Project Overview}

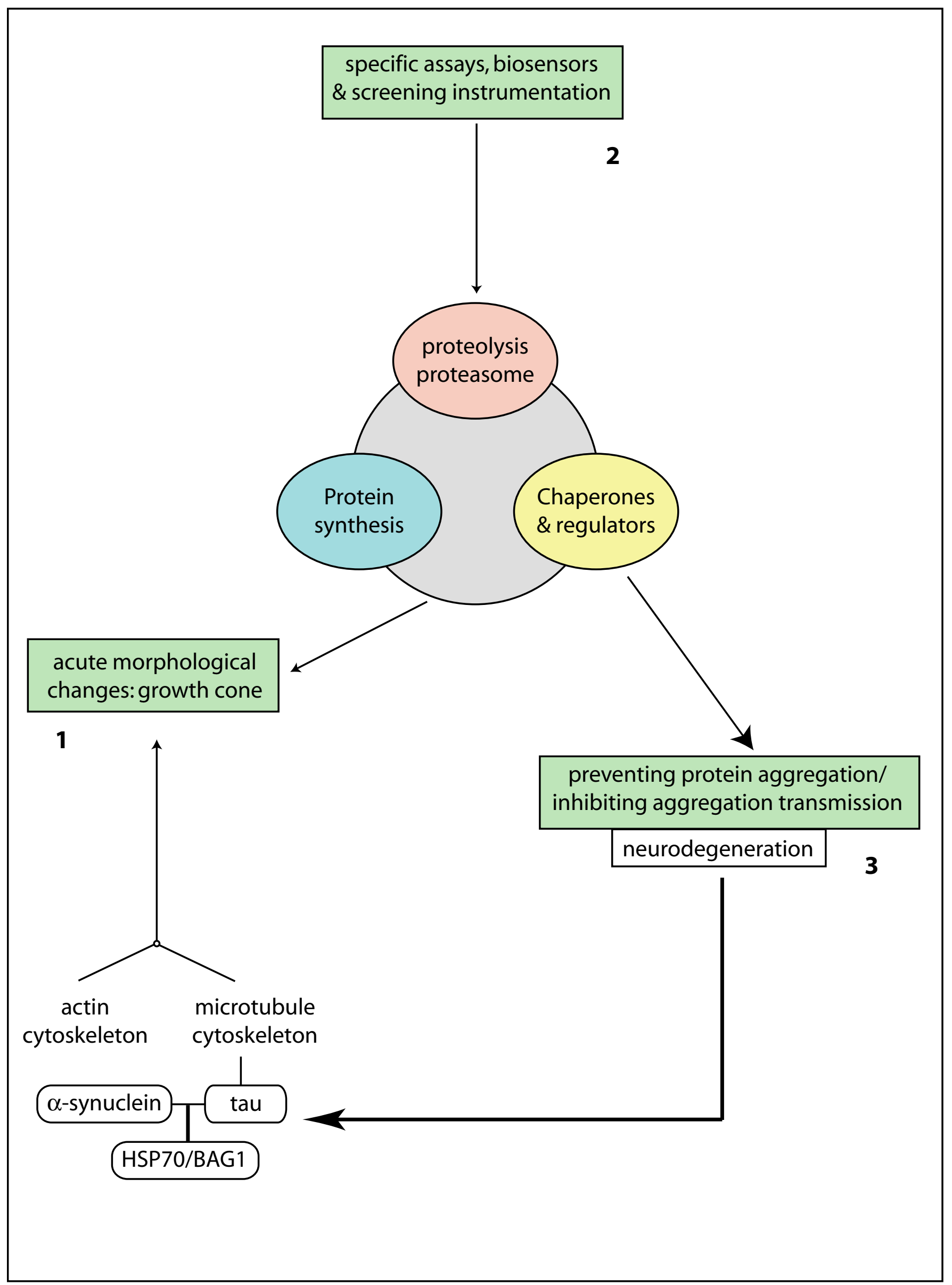




\section{Chapter 4: RESULTS}

\subsection{Generation of biosensors for the study of local protein turnover in the growth cone}

To analyse the growth cone local protein turnover mechanisms, we used FRET based technologies to create biosensor that can detect relevant biochemical reactions in single cell. These biosensors are sensitive toward:

1. Protein transport

2. Protein degradation

3. protein synthesis

4. protein folding

In the following part of the results section, I will discuss about the local protein turnover machinery related biosensor that were created.

\subsubsection{Biosensor to analyse protein transport}

In order to study the role of axonal transport, a biosensor is needed that report the rate and directionality in which protein are transported along axons. We created a sensor that can address local turnover hypothesis with respect to transport. For this we construct an axonal transport marker based on the principle of FRET, which can optically mark specific population of protein with high spatial and temporal resolution. This construct is an Intramolecular FRET tag comprises of CFP donor and 
YFP acceptor flurophore tagged next to each other by a small flexible linker of GlyGly-Arg-Gly-Gly residues and we call this biosensor as FLAP tag (Fluorescence Localization After Photobleaching) named after its properties as FRET sensor

\section{Figure A: FLAP tag}

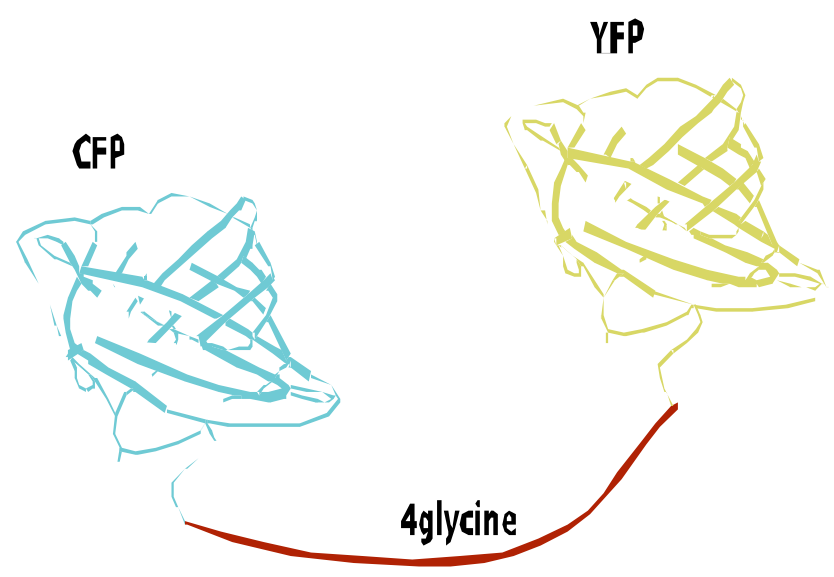

\subsubsection{FRET by FLAP Principle}

FLAP-based FRET sensor works by the principle of acceptor photobleaching. The steps involve in is, by exciting the donor flurophore CFP by $456 \mathrm{~nm}$ argon laser and collecting the fluorescence of both CFP (460-500nm) and YFP (520-560nm) (Figure 1A). Given the sensitized emission from the YFP channel indicates the occurrence of FRET between CFP donor fluorophore and YFP acceptor fluorophore (Figure 1B). By bleaching the YFP molecule by direct excitation at 514nm using 100\% laser illumination for 5 to 10 seconds, recover the fluorescence of CFP which was quenched due to FRET (Figure 1B). By comparing the ratio of CFP over YFP before and after Photobleaching we could get a visual experience of the amount and movement of the bleached protein fraction. The efficient FRET between CFP and 
Figure1: Principle of FLAP tag based transport assay

\section{CFPexcitation}

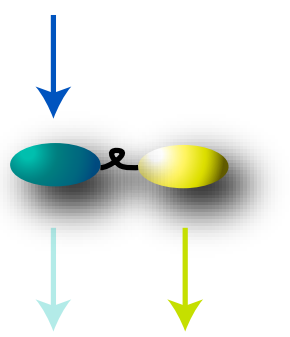

YFPemmision

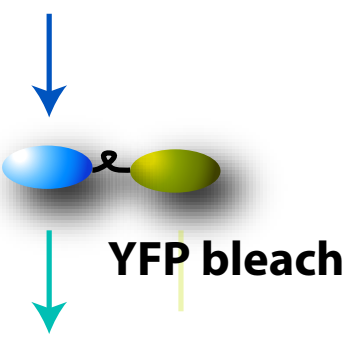

CFP emmision

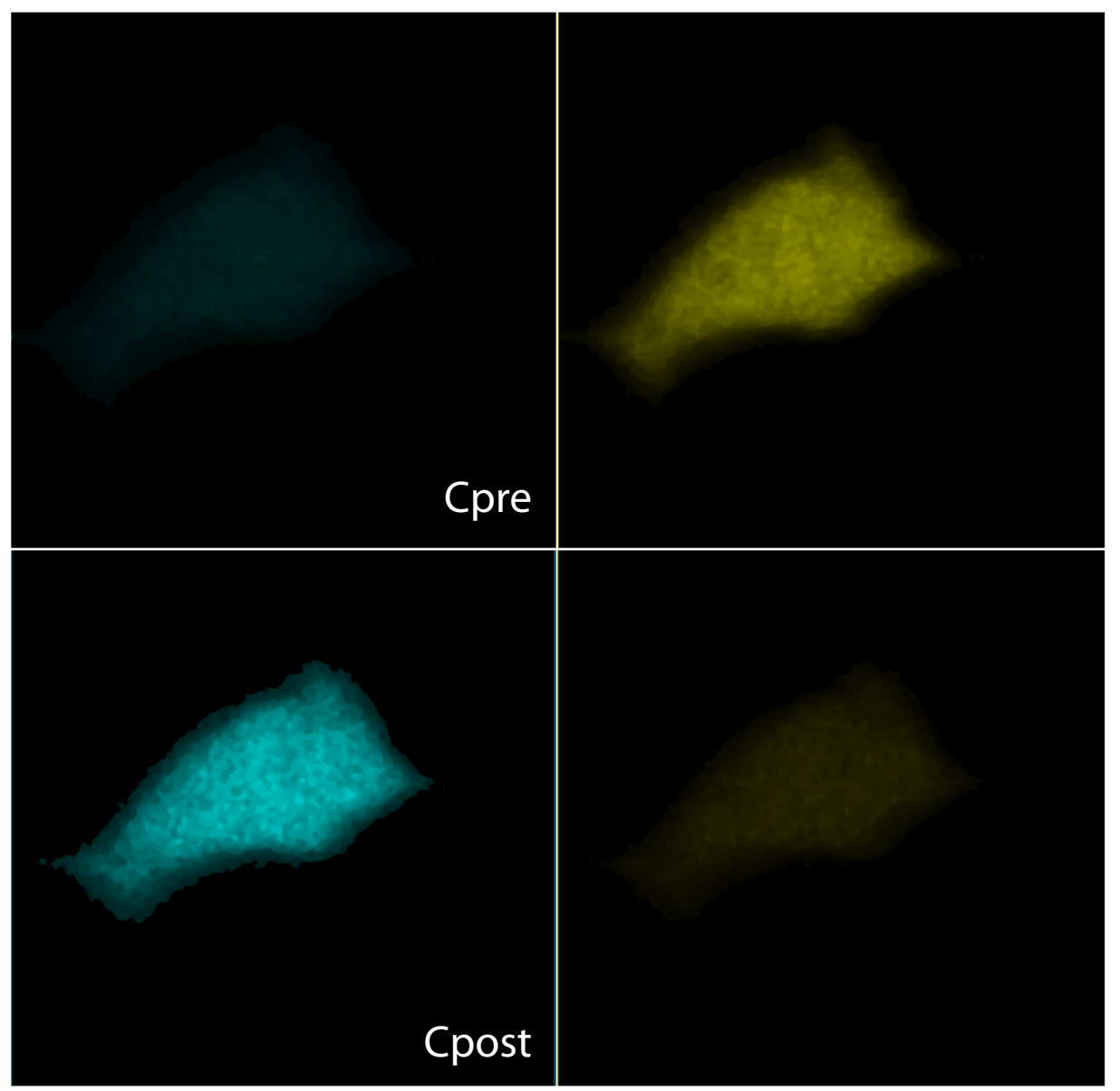

A

\section{FLAP TAG PHOTOBLEACHING EXPERIMENT}

\section{Figure 1: FLAP tag photobleaching experiment for transport assay:}

(A) Figure represents the scheme of acceptor photo-bleaching experiment performed using the CFP-YFP-FPAP tag. (B) Figure representing the FRAP experiments performed in neuroblastoma cells (SHSY5Y) using FLAP tag. Observing YFP emission upon CFP excitation indicates FRET in the FLAP tag. This can further be viewed on removing the YFP emission by bleaching, completely recovers the FRET induced quenching of CFP (Figure B Cpost).The ratio image of CFP over YFP before and after photbleching gives the quantitative measure of FRET efficiency in FLAP tag.(calculated $60 \%$ spproximately). By following the bleached over unleached FLAP tag in a cell gives a measure for directionality of transport ad total protein transported in time. 
YFP is independent of concentration of flurophore because FLAP tag is a single moiety tagged one next to the other. The subsequent emission of CFP and YFP, upon CFP excitation and following the population in time provides us with the possibility to indicate the directionality and rate of protein transport.

\subsubsection{Biosensor to analyse protein ubiquitin machinery}

A current difficulty in genetically expressable FRET pairs is the simultaneous demand for a large spectral overlap between donor emission and acceptor excitation spectra. The other concern is to spectrally seperate the FRET donor and acceptor emission. In this section I will discuss the process by which we created the spectrally seperable, high efficiency FRET sensor, which can efficiently be used as an ideal acceptor for FRET based measurement.

\subsubsection{Generation of REACh by mutating the Yellow Fluorescent Protein (YFP)}

We introduced mutations in the GFP variant YFP to create a dark chromoprotein, which eventually loses its fluorescence emission and maintains the absorption characteristics. Using the point mutation approach by PCR based precise insertion of nucleotides, we replaced the coding sequenc of YFP to mutants. To create a GFP variant with low quantum yield.

The mutations in the GFP RS8/ORG18 mutant was followed up (Kummer, A.D. et $a l$ ), we chose the point mutations at the following positions in the coding sequences of YFP gene Y145W, I148V (Figure A) and subsequently cloned into bacterial 
pRSETA (Invitrogen) and mammalian pEYFPN1 (clontech) vectors for characterising them in bacteria and cell line respectively. We further called these mutant YFP as the (Reasonance Energy Accepting Chromoprotein) REACh1(Y145W) and REACh2 (Y145W, I148V) there after.

\section{Figure A : REACh Mutant}

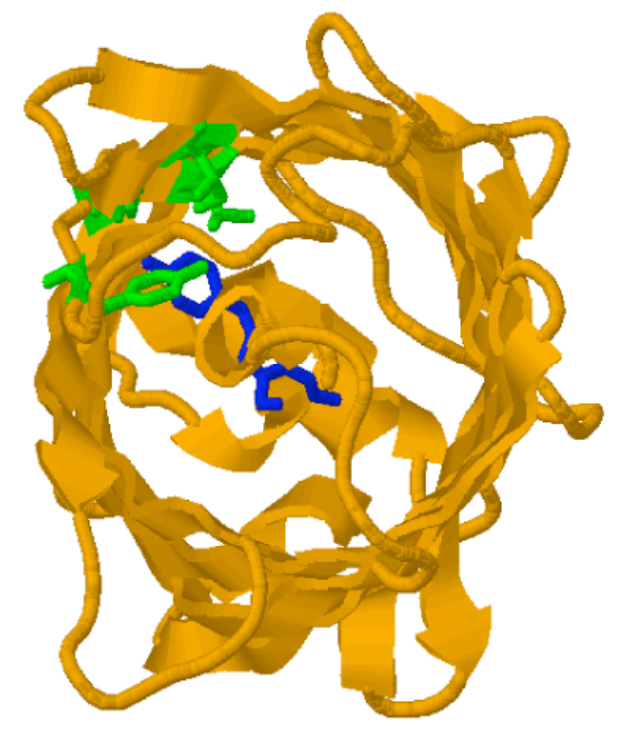

\section{REACh Mutantion and structure}

YFP structure as given by ribbon model shows the beta barrel of 7 parallel beta sheets as arrow (Yellow) and the chromophoric region of GFP resposible for fluorescence emission is shown in ball and stick, representing the atom (Blue). The mutations chosen for creating the chromoprotein, were depicted in atomic configuration by ball and stick model (Green). REACh1 and REACh2 are the mutants of the combination of amino acid residues (Green). Namely, REACh1 Y145W and REACh2 Y145W, I148V

\subsubsection{Spectrofluorimetric and Lifetime analysis Of Mutant Protein}

We examined the YFP mutants, REACH1 and REACH2, for the mutants with reduced quantum yield. Of these mutants, the mutation at position Y145W (REACh1) reduced the fluorescence emission by $98 \%$ and caused a blue shift of the emission maximum from $533 \mathrm{~nm}$ to $530 \mathrm{~nm}, \mathrm{I} 148 \mathrm{~V}$ reduced the emission by $82 \%$ as compared to EYFP. Combination of both mutations reduced the emission by $97 \%$, accompanied by a slight red shift of the emission to $538 \mathrm{~nm}$ (Figure 2A,B). The excitation maximum of the Y145W mutation shows a blue shift from $514 \mathrm{~nm}$ to $495 \mathrm{~nm}$, a blueedge broadening from $50 \mathrm{~nm}$ to $62 \mathrm{~nm}$ half-width, and the spectrum is less structured due to an increase in the low-wavelength shoulder. The excitation spectrum of the 
Figure 2: Spectrofluorimetric characterisation of REACh mutants

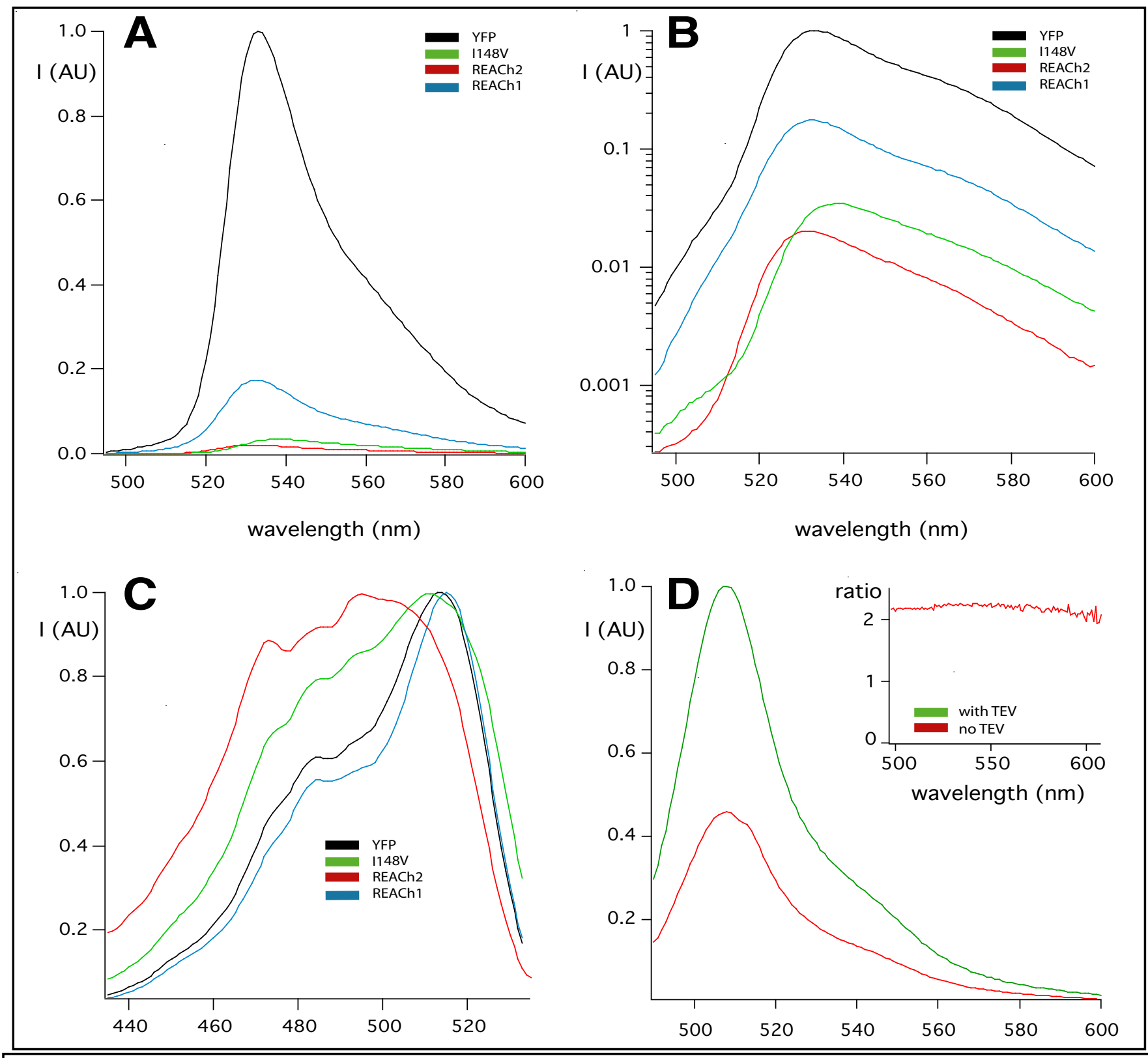

Figure 2. Spectrofluorimetric analysis of recombinant REACh mutants and intramolecular FRET construct. (A) Emission spectra of wild type YFP (black), I148V mutant YFP (blue), REACh1 (red) and REACh2 (green) upon excitation at $490 \mathrm{~nm}$. (B) The emission spectra shown in (A) with the fluorescence intensity (AU) inlog-scale for visualization of the low-intensity REACh1 and -2 mutants. All spectra in $(A, B)$ were corrected for protein concentration. (C) Excitation spectra of wild type YFP (black), I148V mutant YFP (blue), REACh1 (red) andREACh2 (green) with emission set at $540 \mathrm{~nm}$. Spectra were normalized to the excitation maximum of wild type YFP for comparison of spectral shape. Fluorescence intensities are given in arbitrary units (AU). (D) FRET experiment on the GFP-(TEV linker)REACh1 construct. The emission is shown for excitation at $457 \mathrm{~nm}$. The construct was incubated with $10 \mathrm{UTEV}$ protease $/ \mu \mathrm{g}$ protein for $16 \mathrm{~h}$ (green) or without TEV protease (red) Emission spectra are normalized to the maximum emission of the treated sample. Inset shows the ratio of emission spectra of treated overuntreated protein samples. The GFP emission is unquenched by the removal of the acceptor and shows a ratio of 2.2 indicating $55 \%$ FRET. The ratio is not dependent of wavelength, showing that there is no c8ntribution from REACh1 to the emission spectra. 
I148V mutation shows a slight narrowing at the higher wavelengths to $46 \mathrm{~nm}$ halfwidth due to a slight decrease in the low-wavelength shoulder. The combination of both mutations partially corrected the red-shift of the excitation maximum by the Y145W mutation to $510 \mathrm{~nm}$ but still exhibits blue-edge broadening of the excitation spectrum of $65 \mathrm{~nm}$ half-width and decreases the low-wavelength shoulder that was caused by Y145W (Figure 2C). The absorption of these mutants was not significantly affected when compared to YFP and even increased slightly for the double mutant. Y145W absorption was 106\% (at $510 \mathrm{~nm}$ ), I148V absorption was $85 \%$ and the double mutation has an absorption of $120 \%$ of wild-type YFP absorption. These differences most likely reflect folding properties of the recombinant proteins.

\subsubsection{Lifetime analysis on REACh protein in solution at different wavelengths}

Mutant and wild type recombinant proteins purified from bacteria's were subjected to lifetime analysis through time correlated single photon counting measurement coupled to two-photon laser MIRA 2000. The measurements were performed at two different wavelength to excite the proteins samples in their excitation maximum namely, $500 \pm 40 \mathrm{~nm}$ and $550 \pm 40 \mathrm{~nm}$ (Figure 3 ). The corresponding lifetime at different wavelength for the entire mutant protein collections were recorded in table below (Table1)

On the basis of their spectral characteristics, we have chosen the Y145W mutant and the Y145W/I148V double mutant as FRET acceptors for the spectrally similar GFP. A loss of fluorescence is generally caused by a reduced quantum yield, which is in agreement with the short fluorescence lifetimes obtained for the REACh mutants. By extended photon counting of the faint fluorescence from cells expressing extreme amounts of REACh mutants, we determined a lifetime of REACh1 of 319 ps and 
Figure 3: Lifetime analysis of REACh mutants in solution

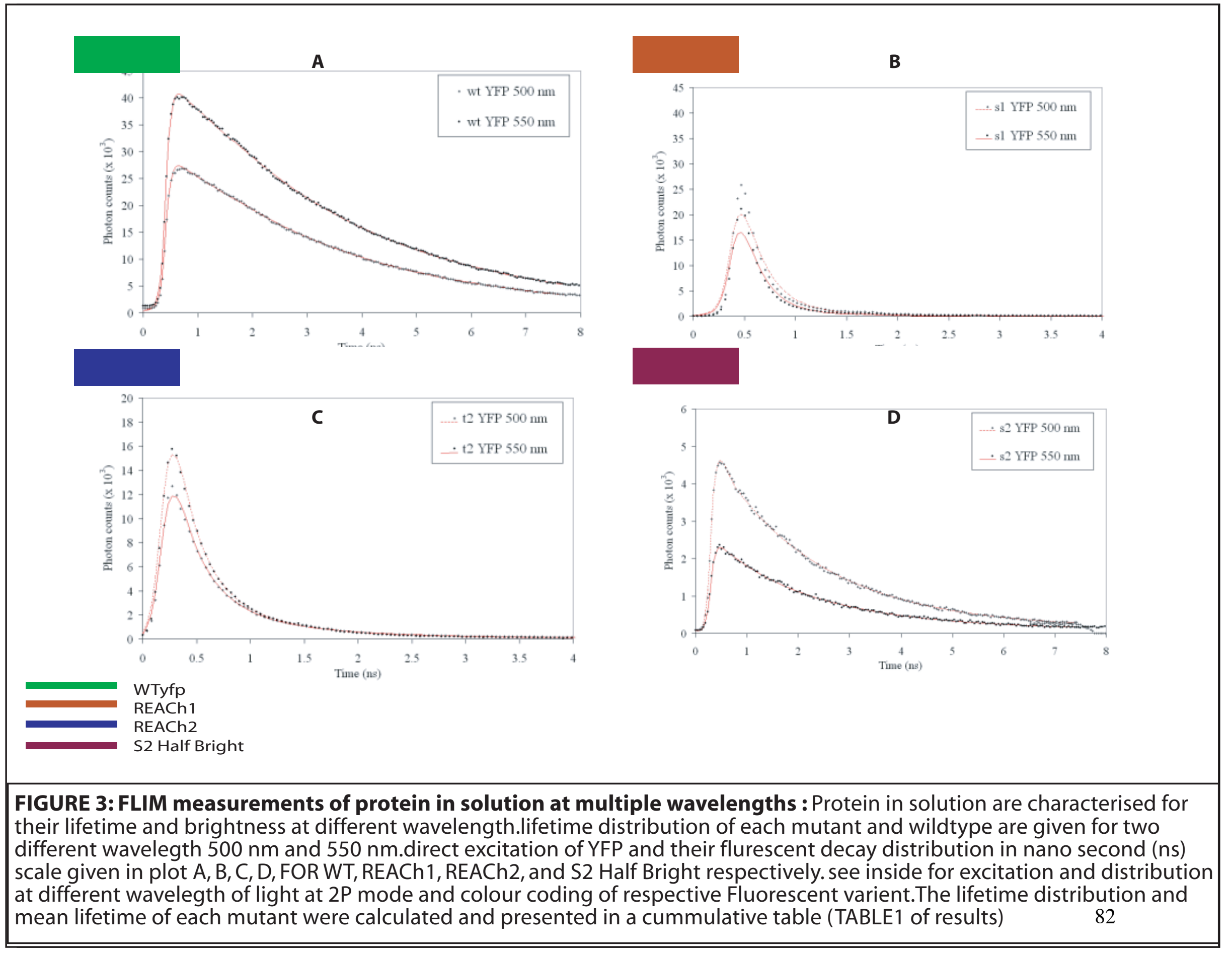


REACh2 of 320 ps (Figure $2 \mathrm{~B}, \mathrm{C}$ ), as compared to the lifetime of $2.9 \mathrm{~ns}$ for YFP (Figure $3 \mathrm{~A}$ ) and half bright S2 mutant of $1.5 \mathrm{~ns}$ and was also confirmed by the lifetime measurement of REACh1 protein mammalian cells (Figure 4). In the case of the RS8/ORG18 GFP mutant, a quantum yield reduction was showed to be caused by the occurrence of fast internal conversion (Kummer, A.D. et al). This is most likely explained by mutation-induced spatial relaxation of the beta-barrel inner core, which allows the fluorophore to release its excited state energy to its environment, and we assume that the same mechanism is responsible for the loss of fluorescence in REACh. From the obtained spectra, the Förster distance $\left(\mathrm{R}_{0}\right)$ of the REACh1/2-GFP FRET pairs, (the distance at which $50 \%$ of the molecules undergo FRET), was calculated (Harpur, A.G. et al a laboratory manual). The $\mathrm{R}_{0}$ distance for the $\mathrm{Y} 145 \mathrm{~W}$ mutant is $5.6 \mathrm{~nm}$, identical to the $\mathrm{R}_{0}$ for the GFP-YFP pair, $5.4 \mathrm{~nm}$ for the $\mathrm{I} 148 \mathrm{~V}$ mutant and $5.9 \mathrm{~nm}$ for the $\mathrm{Y} 145 \mathrm{~W} / \mathrm{I} 148 \mathrm{~V}$ double mutant. The higher $\mathrm{R}_{0}$ value for REACh2 is due to its broader excitation half-width, thereby causing a larger spectral overlap with GFP emission. REACh1 loses this advantage due to the concomitant blue shift of the excitation maximum (Table 2).

We demonstrate the usefulness of this novel REACh-GFP FRET pair by fluorescence spectroscopy on a recombinant intramolecular FRET construct. To this end, GFP and REACh1 were fused by a peptide sequence that is specifically recognized by the TEV protease. FRET, present in the intact construct was lost upon cleavage of this linker sequence. The GFP emission increases 2.2-fold upon incubation with TEV protease, indicative of the presence of 55\% FRET in the intact construct (Fig. 2D). This increase was not present upon TEV treatment of a construct lacking the TEV site. Importantly, the emission of the TEV construct was increased two-fold over the entire emission spectrum (see the ratio of emission spectra in the insert Figure 2D), showing that the emission originates exclusively from the GFP donor without contribution of residual YFP emission. 
Figure 4: Lifetime analysis of REACh mutants in mammalian cells

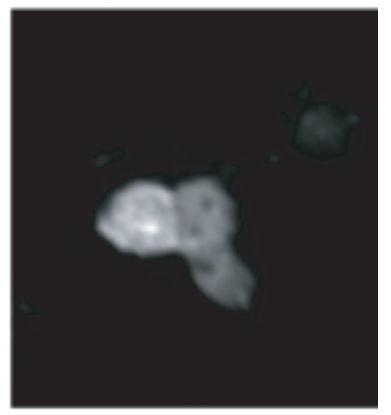

A

REACh1 in MCF-7 cells 320PS

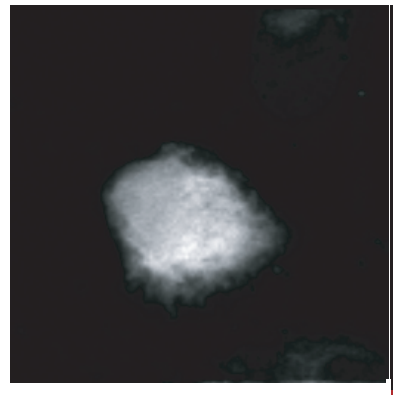

C

REACh2 in MCF-7 cells 320PS

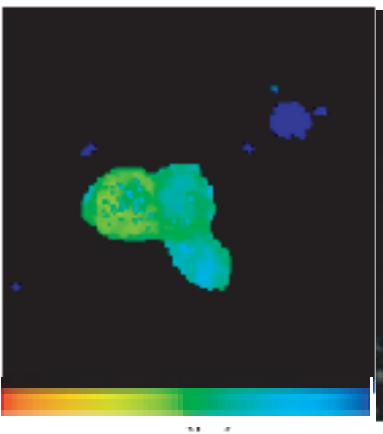

ns

0.8

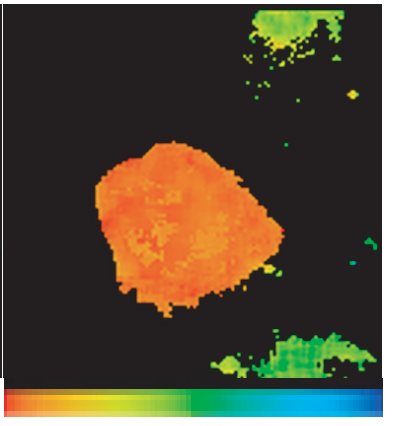

0.5

ns

2.2

B
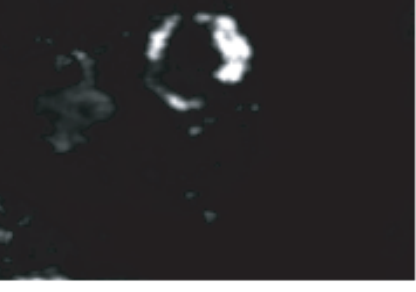

B

REACh1 ubq in MCF-7 cells 320PS

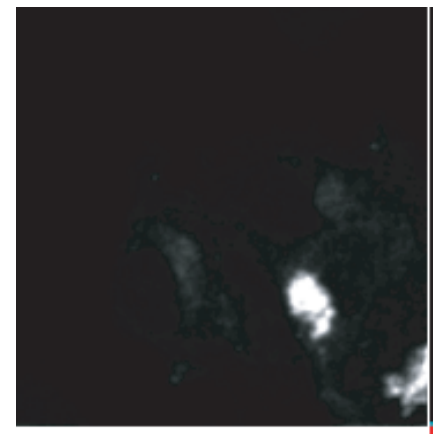

D

0.5

ns

Figure 4: Fluorescent Lifetime analysis of REACh mutants

(A)Fluoresent emission distribution of REACh1 shows a homogenous distribution in MCF-7 cells and the corresponding lifetime image in a pseudo colur map.(B) Fluorescent emission imagee of REACh1-ubiquitin shows a aggregated worm like distribution of protein around the perinuclear structures with no change in lifetime when compared to REACh without ubqiquitin, shows no change in lifetime by tagging ubiquitin (C) FLIM images of REACh2 mutant witout ubiquitin tag show similar fluorescence distribution to REACh1(A) with no change in lifetime compared to the REACh2-ubiquitin shown in(D). Green patches around the cell with lower lifetime is due to low signal to noise ratio contributes to lifetime 
Table1. Lifetime analysis with protein in solution

\begin{tabular}{|c|c|c|}
\hline Wavelength2photon(nm) & $\begin{array}{c}\text { Mean Lifetime } \\
@ 500 \pm 40\end{array}$ & $\begin{array}{c}\text { Mean Lifetime } \\
@ 550 \pm 40\end{array}$ \\
\hline Mutant protein Name & 3.13 & 3.26 \\
\hline WT & 0.32 & 0.4 \\
\hline S1 & 1.48 & 1.23 \\
\hline S2 & 0.26 & 0.23 \\
\hline T2 & & \\
\hline
\end{tabular}

Table2. Spectral characteristics of REACh mutant's

\begin{tabular}{|l|l|l|l|l|l|l|l|}
\hline Mutation & Name & $\begin{array}{l}\text { Excitation } \\
\text { maximum } \\
(\mathrm{nm})\end{array}$ & $\begin{array}{l}\text { Excitation } \\
\text { bandwidth } \\
\text { FWHM } \\
(\mathrm{nm})\end{array}$ & $\begin{array}{l}\text { Emission } \\
\text { maximum } \\
(\mathrm{nm})\end{array}$ & $\begin{array}{l}\text { Relative } \\
\text { fluorescence }\end{array}$ & $\begin{array}{l}\text { Relative } \\
\text { absorption }\end{array}$ & $\begin{array}{l}\text { R0 } \\
\text { GFP } \\
\text { Donor } \\
\text { (nm) }\end{array}$ \\
\hline Wild type & EYFP & 514 & 50 & 533 & 1.00 & 1.00 & 5.6 \\
\hline I148V & Single2 & 515 & 46 & 532 & 0.18 & 0.85 & 5.4 \\
\hline Y145W & REACh1 & 495 & 62 & 530 & 0.02 & 1.06 & 5.6 \\
\hline $\begin{array}{l}\text { Y145W+ } \\
\text { I148V }\end{array}$ & REACh2 & 510 & 65 & 538 & 0.03 & 1.20 & 5.9 \\
\hline
\end{tabular}

(FWHM: full width at half maximum). 


\subsubsection{A REACh-based single-cell FRET sensor for protein ubiquitination}

Since our dark REACh mutants no longer emit fluorescence, as it avoids the spectral blead through REACh mutants can be used as an ideal acceptors for FRET microscopy with GFP. We designed a FRET assay for the activity of the protein ubiquitination machinery that relies on the interaction between GFP- and REAChlabeled proteins inside cells. For this, the GFP donor was fused to the efficient PEST ubiquitination substrate (GFP-PEST) and was co-expressed with REACh2-labeled ubiquitin. FRET occurs when REACh-ubiquitin molecules are covalently attached to GFP-PEST. This poly-ubiquitination modification then targets the GFP-PEST to active proteasomes where the protein is digested (Figure 5,6,7).

\subsubsection{Biochemical and immuno-cytochemical characterisation of REACh protein ubiquitination}

REACh-ubiquitin was accepted as a substrate for the ubiquitination machinery, since western blotting of protein samples from cells, expressing REACh2-ubiquitin $(\sim 35$ $\mathrm{kDa}$ ) showed high-molecular weight adducts (ladder with prominent enrichment at $\sim 250 \mathrm{kDa}$ and higher) with anti-ubiquitin (Fig. 5E) and anti-GFP antibodies (Fig. 5F) and there was also a fraction of proteolytically liberated REACh chromoprotein $(\sim 27$ $\mathrm{kDa}$ ) which escaped complete proteasomal degradation (Fig. $5 \mathrm{~F}$ ). The band at $\sim 8 \mathrm{kDa}$ in (Fig. 5E) represents endogenous ubiquitin. The GFP-PEST was distributed throughout the cell cytosol and, due to its small size, passively enters the nucleus. In addition, small punctate clusters with higher intensity can be observed in the perinuclear region (Figure 5A). The latter staining most likely indicates that the GFP- 
Figure 5 : Antibody colocalisation of REACh ubiquitination of GFP-PEST

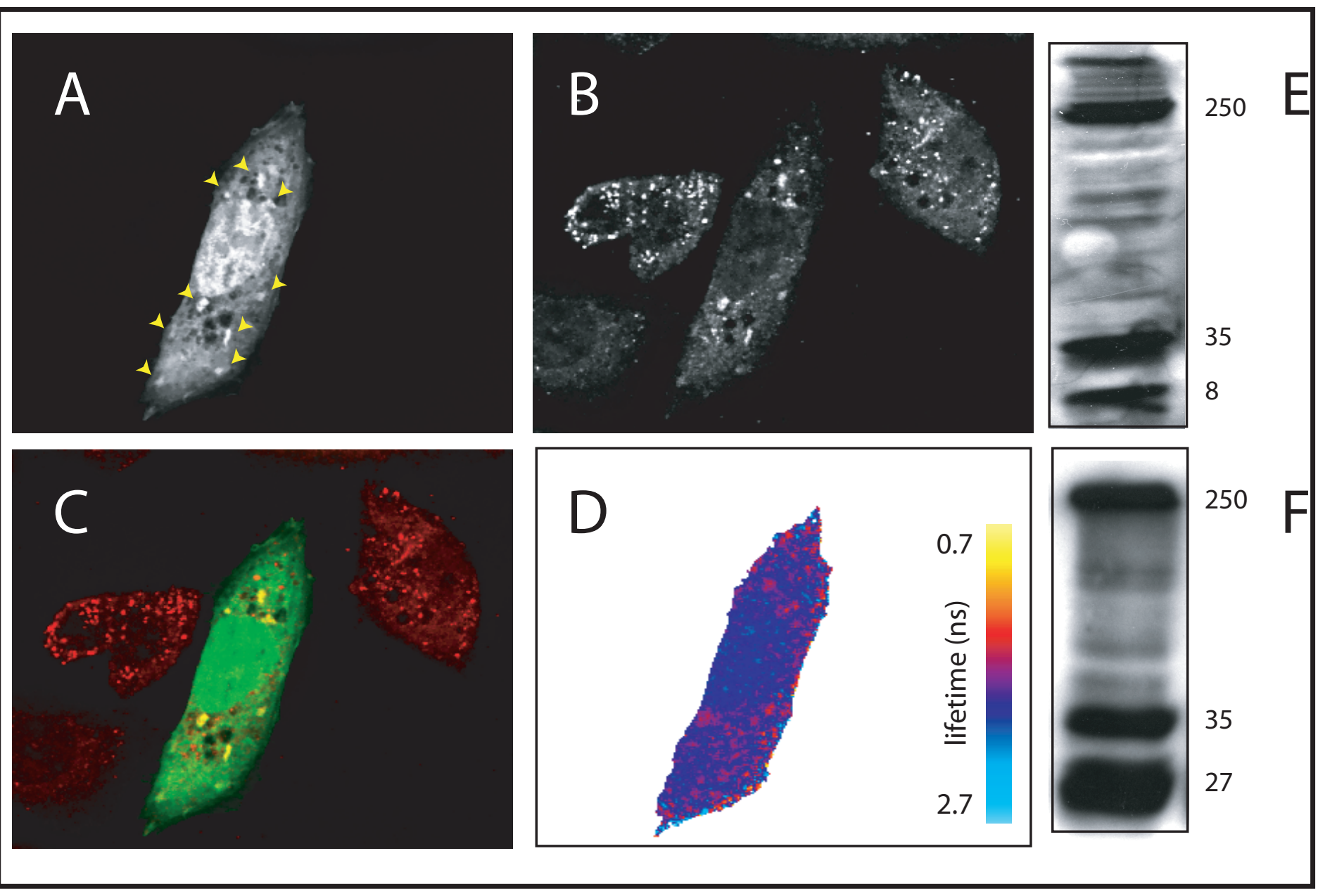

\section{Figure 5: REACh-ubiquitination of the GFP-PEST substrate in cells.}

(A) Fluorescence emission distribution of GFP-PEST shows a homogeneous distribution in the cytoplasm in addition to a passive enrichment in the nucleus and punctate/wormlike higher intensity structures throughout the cytoplasm (arrowheads). (B) immunofluorescence staining with anti-ubiquitin antibodies identifies the high intensity GFP structures in (A) as ubiquitin-rich particles, most likely proteasomes. Shown are cells co-expressing GFP-PEST andREACh2-ubiquitin, 4 surrounding cells do not express the GFP-PEST. (C) Overlay of GFP-PEST (green channel) and REACh2-ubiquitin (red channel) indicates co-localisation (yellow signals) in proteasomal structures. (E) FLIM image of the same cell using 2-photon TCSPC imaging shows the presence of low-lifetime signals in cytoplasmic structures, indicative of FRET between GFP-PEST and conjugated REACh-ubiquitin. (F) Western blot of cells expressing GFP-PEST and REACh-ubiquitin with anti-ubiquitin antibody showing free ubiquitin ( 8 kDa), REACh-ubiquitin ( $\sim 35 \mathrm{kDa})$ and high-molecular weight adducts (ladder with prominent enrichment at $\sim 250 \mathrm{kDa}$ and higher). (G) Western blot of cells expressing GFP-PEST and REACh-ubiquitin with anti-GFP antibody showing free REACh ( 27 kDa), REACh-ubiquitin ( 35 kDa) and high-molecular weight adducts (ladder with prominent enrichment at $\sim 250 \mathrm{kDa}$ ). 
PEST kinetically accumulates in proteasomes during its degradation. The colocalisation of anti-ubiquitin immunoreactivity (Fig. 5B,C) in these clusters confirms the accumulation of ubiquitinated GFP-PEST and further establishes these structures as likely proteasomes. Note the comparable distribution of ubiquitin immunoreactivity in untransfected neighboring cells (Figure 5B), which shows that the distribution of proteasomes in cells expressing GFP-PEST is not disturbed. To demonstrate that this co-localisation represents covalently ubiquitinated GFP-PEST, we measured FRET between GFP-PEST and REACh2-ubiquitin by FLIM. As can be seen in (Figure 5D), punctate structures with clearly reduced fluorescence lifetimes (1.5 to $1.7 \mathrm{~ns})$ can be observed in the cytoplasm (as visualised in the pseudo color loolup table for lifetime), indicative of the occurrence of FRET at these sites. Fluorescence emission was never detected by two-photon microscopy of cells expressing only REACh-ubiquitin or unfused REACh mutants under the imaging conditions used by us for the detection of ubiquitination.

\subsubsection{Lifetime analysis of REACh-FRET sensors in multiple spectral windows}

One major advantage of REACh is that FLIM measurements can be performed on all emitted photons as they only originate from the donor. Therefore, we compared the FLIM analysis of FRET in two spectral emission windows to confirm again the absence of REACh fluorescence contribution to the donor lifetime measurements. (Figure 6) shows the fluorescence lifetime distribution of the FRETting GFPPEST:REACh2-ubiquitin complex using a GFP narrow band-pass emission filter in front of the single photon detector. This was compared with a measurement where an emission filter was omitted (Fig. 7) to sample all emitted photons. Reduced lifetimes 
can be seen in peri-nuclear structures (Figs. 6A,D, 7A,D) that are similar to the ubiquitin-containing structures in Fig. 5, showing the accumulation of ubiquitinated GFP-PEST in proteasomes. In fact, with the localisation of these proteasomes revealed by the reduced lifetime, it should be possible to recognize these structures also in the GFP fluorescence intensity images shown in panel A of (Figures 6 and 7), even in the background of homogeneous cytoplasmic GFP-PEST. Furthermore, a slight reduction of the fluorescence lifetime can be observed in the cytoplasm, indicative of ubiquitination of GFP-PEST that has not yet been targeted to the proteasomes. In contrast, avoiding of REACh-ubiquitin coexpression produced GFPPEST emission with typical non-FRETing GFP lifetime, demonstrating that proteasomal processing did not affect the GFP lifetime (Figs. 6B,E, 7B,E).

A contamination with possible residual short lifetime YFP-REACh fluorescence would cause an obvious additional global reduction in lifetime in the measurement lacking the GFP emission filter. In agreement with our spectroscopic measurements, the results are identical (Fig. 6,7), indicating the exclusive dependence of the measurement on GFP emission. This is especially clear from the typical non-FRET GFP lifetimes that could be observed in the control sample where PEST-GFP is substituted with eGFP (Figs. 6C,F, 7C,F).

To further increase the stringency of these control measurements, we have selected examples from a small population of cells, that shows aggregates in the GFP emission window (most extreme case shown in (Fig. 6C). We reasoned that possible coclustering of high levels of GFP and REACh-ubiquitin in these aggregates would provide an extreme condition in which some of the residual fluorescence of REACh 


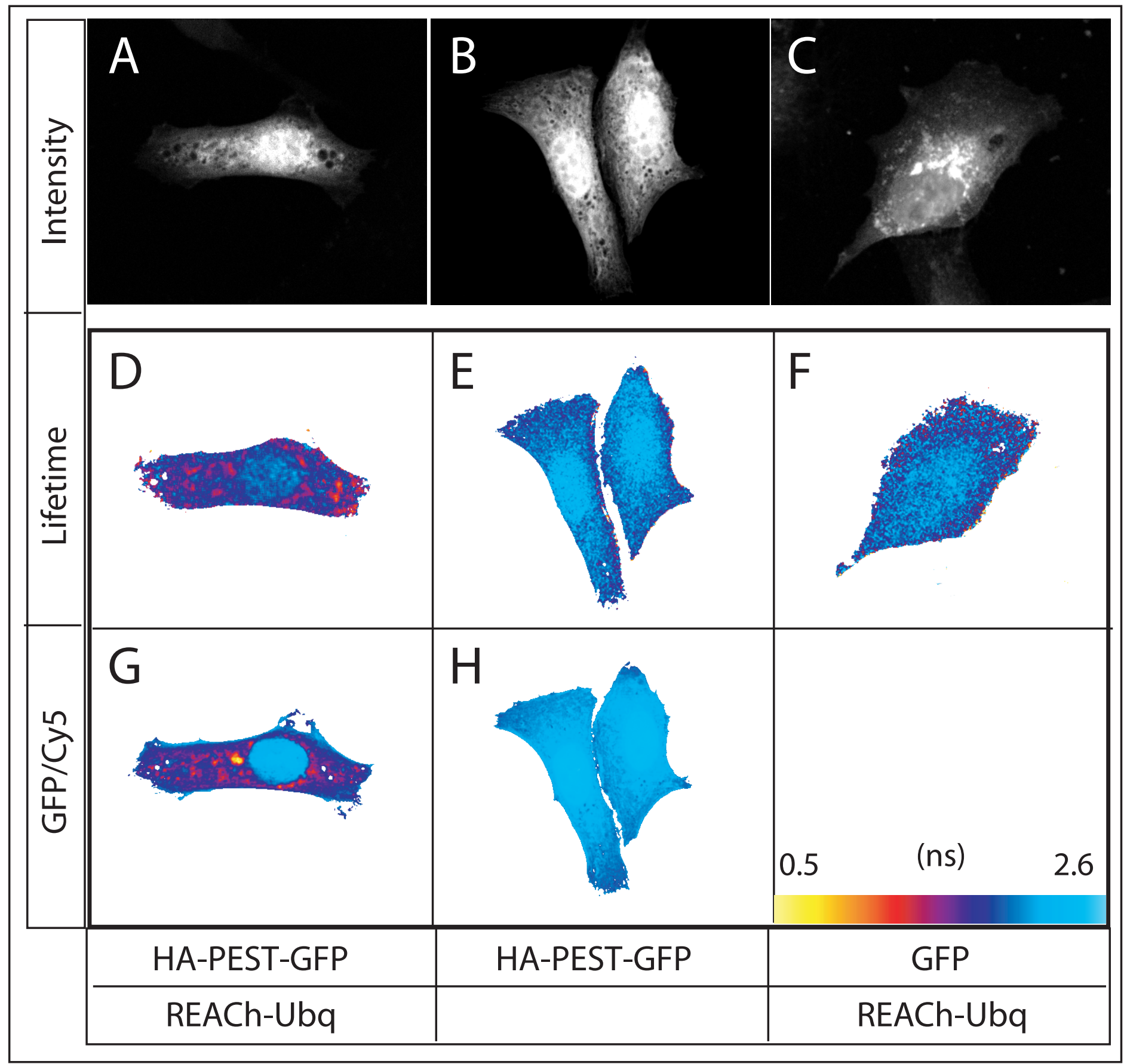

Figure 6: FRET measurement of GFP-PEST REACh-ubiquitination by FLIM and FqRET.

Fluorescence lifetimes were determined using time-correlated single-photon counting on a 2-photon microscope with fluorescence detection without any emission filter. FqRET was performed on a confocal microscope. (A-C) GFP fluorescence intensity images of HA-GFP-PEST $(A, B)$ or non-PEST GFP $(C)$ in the presence of REACh2-ubiquitin $(A, C)$. (D-F) Corresponding lifetime maps showing the presence of ubiquitination of GFP-PEST in (A) as judged by lower GFP lifetimes. The cells expressing HA-GFP-PEST without REACh-ubiquitin (E) and non-PEST GFP in the presence of REACh-ubiquitin (F) show similar homogeneous lifetimes. Corresponding FqRET ratio images of GFPand Cy5 emission for cells expressing HA-PEST-GFP in the presence $(D, G)$ and absence $(F, H)$ of REACh-ubiquitin. Also here, a lower lifetime (D) and GFP/Cy5 ratio (G) can be observed in the cytoplasm. From the lifetime values, again FRET efficiencies between GFP-PEST and REACh2-ubiquitinwere calculated to range from $25-40 \%$, confirming that the lifetime results are not influenced by possible residual REACh emission. Warm colors indicate high FRET (low lifetime and ratio), light blue colors indicate the absence of FRET.. 


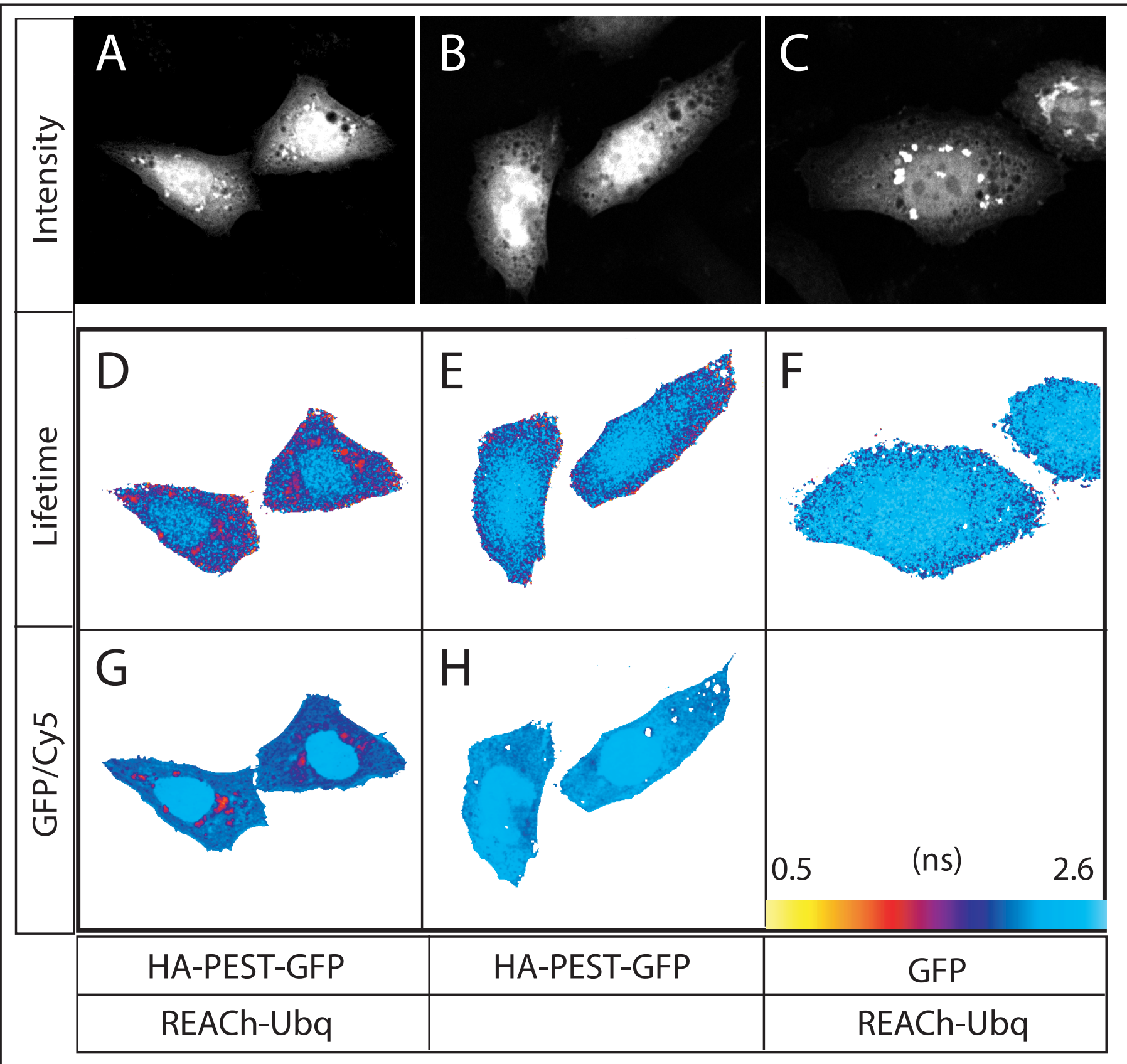

Figure 7: FRET measurement of GFP-PEST REACh-ubiquitination by FLIM and FqRET.

Fluorescence lifetimes were determined using time-correlated single-photon counting on a 2-photon microscope with fluorescence detection through a GFP emission band-pass filter. FqRET was performed on a confocal microscope. (A-C) GFP fluorescence intensity images of HA-GFP-PEST $(A, B)$ or non-PEST GFP (C) in the presence of REACh2-ubiquitin (A,C). (D-F) Corresponding lifetime maps showing the presence of ubiquitination of GFP-PEST as judged by the lower lifetime in punctate structures, indicative of active proteasomes (A). The cells expressing HA-GFP-PEST without REACh-ubiquitin (E) shows a homogeneous lifetime (E) that is comparable to the lifetime of non-PEST GFP in the presence of REACh-ubiquitin (F) demonstrating that the GFP fluorescence lifetime is not affected by proteasomal targeting due to the PEST sequence and the absence of lifetime contribution of possible residual REACh fluorescence. Corresponding FqRET ratio images of GFP and Cy5 emission for cells expressing HA-PEST-GFP in the presence $(D, G)$ and absence $(F, H)$ of REACh-ubiquitin show the same structures as identified by lower lifetimes by FLIM. Small differences are explained by the fundamentally different imaging methods: 2-photon excitation for (D-F) and 1-photon confocal imaging for $(\mathrm{G}, \mathrm{H})$. Furthermore, the apparent higher ratio in the nuclear areas is caused by underrepresented $\mathrm{Cy}-5$ immunostaining due to lower penetration into the chromatin. Note that in addition to the punctate FRET structures, a lower lifetime (D) and GFP/Cy5 ratio (G) can be observed in the cytoplasm that is indicative of cytosolic REACh ubiquitination of PEST-GFP. From the lifetime values FRET efficienciesbetween GFP-PEST and REACh2-ubiquitin were calculated to range from 25-40\%. Warm colors indicate high FRET (low lifetime and ratio), light blue colors indicate the absence of FRET. 
could be detected by the lowered lifetime. In fact, in none of the control cells and the selected cells shown in panel $\mathrm{C}$ of figures 6 and 7 could any significant lifetime reduction be observed. This might be due to the fact that overexpression of GFP may also be targetted to proteasome clusters for clearence, which obviously have no or little interaction figure $(6 \mathrm{C}, \mathrm{F}, 7 \mathrm{C}, \mathrm{F})$.

\subsubsection{Donor Photobleaching kinetics as a indicator for the occurence of FRET}

The use of the REACh acceptor proteins implies a FRET detection method based on donor fluorescence properties. Besides FLIM, REACh acceptors can also be used for intensity-based FRET detection. FRET protects fluorophores against photobleaching since FRET reduces the lifetime of the excited state, on which the photochemical reaction of photobleaching acts. FRET can therefore be measured by analysis of donor fluorescence photobleaching kinetics (Jovin, T.M et al) in a conventional microscope. Figure 3 shows a cell expressing GFP-PEST and REACh1-ubiquitin (Figure 3A).

The bleaching rate of GFP-PEST under continuous illumination resulted in a lower bleaching rate as can be clearly observed in peri-nuclear structures that correlate to high-intensity GFP-PEST structures representing active proteasomes (Figure 6A, see also Figure $4 \mathrm{G}, \mathrm{J})$. This results in a higher relative amount of residual GFP fluorescence in these structures (Figure 8C) during the photobleaching procedure. Cells expressing GFP-PEST without REACh-ubiquitin (Fig. 8B) show a homogeneous bleaching rate (Figure 8D). We compared the bleaching kinetics of the bleach-protected structures in Fig. 8A (Figure 8A box 1, 8E O), the FRET situation, with a control region in the same cell (Figure $8 \mathrm{~A}$ box 2, 3E $\mathbf{0}$ ) and a region in a control cell lacking the REACh-ubiquitin acceptor (Figure 8A box 3, 8E $\square$ ). As can be seen in Fig. 8E, only the proteasomal structures show a significantly slower 
Figure 8: Donor photobleaching analysis of REACh ubiquitination

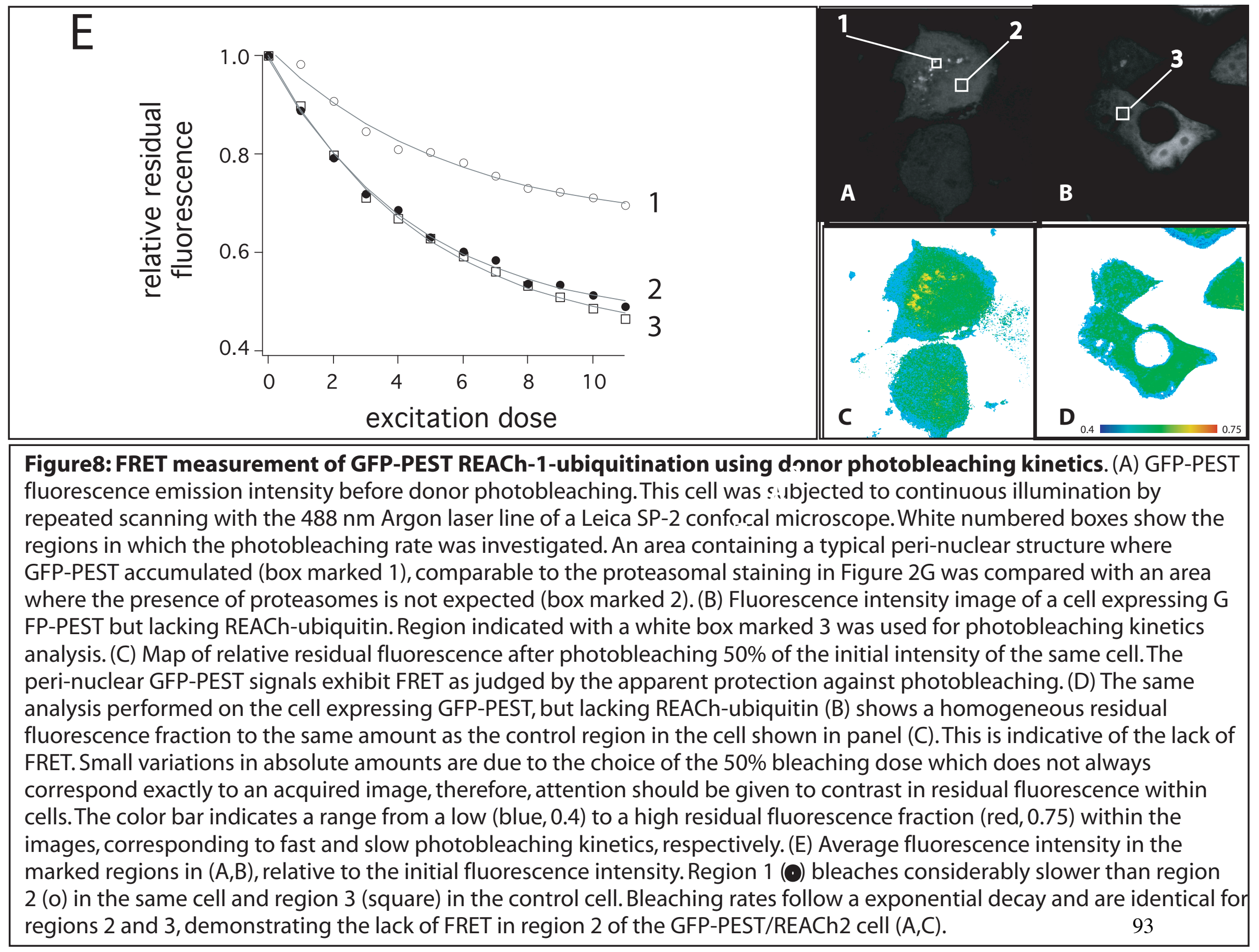


photobleaching rate. This contrast in bleaching kinetics between structures exhibiting FRET in a non-FRET background allows the visualisation of the occurrence of FRET, as in Fig. 8C.

\subsubsection{FqRET Fluorescence quenching Reasonance Energy Transfer}

FqRET is a alternative tool for measuring FRET between two flurophores when they interact. It follows the principle of fluorescence quenching of donor by FRET, which can be directly correlated with the intensity of the reference fluropore that emits far from the FRET spectral window. Using the intensity of donor and reference flurophore and the corresponding ratiometric analysis of donor fluorescence over reference fluorescence. Figures 6 and 7 also show an additional and independent donor fluorescence intensity-based method for the determination of FRET (Figs $6 \mathrm{G}, \mathrm{H}, 7 \mathrm{G}, \mathrm{H})$. These two sets of experiments were performed on HA-tagged GFPPEST in the presence of Cy5-immunofluorescence detection of the HA tag. The Cy5 emission is well-separated from the absorption wavelength of REACh by its spectral high-wavelength position and its emission is therefore not affected by FRET coupling in this system.

This allows the Cy5 signal to be used as an optically inert concentration reference for the quantitation of FRET-quenched GFP-PEST fluorescence emission by division of the GFP emission upon GFP excitation by the Cy5 emission upon Cy5 excitation. The reduced GFP-PEST emission due to FRET with the REACh-ubiquitin, is detected by a decrease of the emission ratio in this concentration-independent measurement. As can be seen from Figures 6D,G and 7D,G, the low lifetime clusters match the reduced GFP/Cy5 emission ratio in the same cell. The presence of ubiquitinated PEST-GFP in 
the cytoplasm can also be observed in the emission ratio maps in the presence and absence of REACh2-ubiquitin (Figs. 6G, 7G and 6H, 7H respectively). Small deviations are caused by the difference in focal size and position between the lifetime images (Figs 6D, 7D: 2-photon microscopy) and the ratio images (Figs 6G, 7G: confocal microscopy). The increased emission ratio in the nucleus is caused by incomplete penetration of the anti-HA antibody in the dense chromatin structure of the nucleus. Cy5 does not give rise to significant FRET with the GFP, as can be seen by comparison of fluorescence lifetimes in panel E of Figures 6 and 7 where a nonPEST GFP served as inactive REACh-ubiquitination substrate.

The lifetime maps of GFP lacking the PEST sequence, when co-expressed with REACh2-ubiquitin (Figures 6,7 panel F) and REACh1-ubiquitin (not shown), show a homogeneous distribution of approximately $2.4 \mathrm{~ns}$ that is typical for EGFP. We have purposefully selected GFP-expressing cells that exhibit a clustered appearance as a result of high expression levels (most extreme case shown in Figure 6C) to be able to show the selectivity and concentration-independence of the assay. No lifetime reduction could be observed in these clusters, indicative of the absence of covalent ubiquitination. These high-intensity GFP clusters either represent insoluble aggregates and/or proteasomal structures clearing the cell from an excess of GFP. If REACh coclusters in these structures, then their local high concentration by itself is not sufficient to contaminate the GFP lifetime by their possible residual fluorescence. More importantly, they do not exhibit FRET, indicating that the FRET signals observed for PEST-GFP and REACH-ubiquitin is not likely explained by simple coinclusion in proteasomes. The same lifetime distribution is obtained from GFP-PEST fluorescence in the absence of REACh-ubiquitin (Figures 6,7 panel E). This set of 
experiments proves that the measured fluorescence lifetimes correspond to GFP and that a possible contribution of REACh residual fluorescence can be neglected under the conditions used. In conclusion, we demonstrate by different methods the suitability of the REACh acceptor in determining FRET between proteins in a physiologically relevant setting. In its current use, the ubiquitination assay reports on the local activity of the ubiquitination machinery, but can easily be converted in a cell-based screening assay for candidate protein substrates for ubiquitination. Although the REACh acceptor is not visible in cells, the meaningful readout of FRET measurements is the visualisation of the donor-acceptor complex. This is inferred from the occurrence of FRET as judged from the donor fluorescence properties. In fact, the acceptor is commonly present in excess over the donor, to saturate the donor binding event for optimal and quantitative detection of the interaction. The localisation of the acceptor was established in a separate experiment with antibody against Ubiquitin tagged to the acceptor flurophore, which is practically invisible. It can be visualised with a reference fluoroprotein far apart from the donor and acceptor emission for example CY5.1 fluorescent dyes.

\subsubsection{Biosensor to analyse protein folding and chaperone activity}

\subsubsection{Creation of folding mutant and Recombinant protein characterisation}

The chaperone dependent folding activity sensor was identified from a random mutagenesis screen of YFP by visual inspection of the bacterial phenotype of the colonies. The Bacterial pRSETB EYFP gene was mutated by using random mutagenesis Kit with XL1red strain (Stratagene). This experimental step uses the mutated DNA polymerase, which lack the proof reading activity. Plasmid DNA were made from total colonies and re-transformed into BL21DE3 (Stratagene) bacterial 
expression strain. Individual colonies were then inspected manually for their fluorescent phenotype. The phenotype indicative of folding impairment is a colony with combined fluorescent and non-fluorescent bacteria. Due to the expressiondependent upregulation of the bacterial chaperone program (Jürgen et al. 2000) and the continuous bacterial division in the colony, one or more fluorescent "zigzag" lines, corresponding to a contorted fluorescent colony slice is formed, that can easily be recognized by eye (See Figure 9A). Based on this phenotype, 17 colonies were picked from \pm 15.000 colonies screened and plasmid DNA was isolated. These 17 cDNAs were re-transformed into B121 bacteria for detailed and statistical evaluation of their phenotype. Of these 17 candidates, 5 were selected that showed significant proportions of fluorescent protein, similar folding between colonies, an early folding response (streaks originate from the core of the colony) and recovery of high fluorescence intensities upon folding. These 5 candidates were then subjected to bacterial chaperone induction by a low-temperature ethanol shock (Thomas et al. 1996) in liquid culture. The purified protein fractions were then analysed using a Pharmacia Biotech Ultrospec-3000 spectrophotometer and a PTI Quantamaster spectrofluorometer.

The typical and obvious heterogeneous distribution of fluorescence in the colonies expressing foldase-sensitive mutants (Fig. 9A) is explained by the spontaneous upregulation of the bacterial chaperone program due to the presence of high concentrations of unfolded YFP in the bacterial cytoplasm (Jürgen et al. 2000). It is known that GFP folding in vivo involves chaperones and that different GFP variants follow different folding trajectories (Sacchetti et al. 2001). 
Figure 9: Characterization of cdYFP folding efficiencies in bacterial and mammalian cells

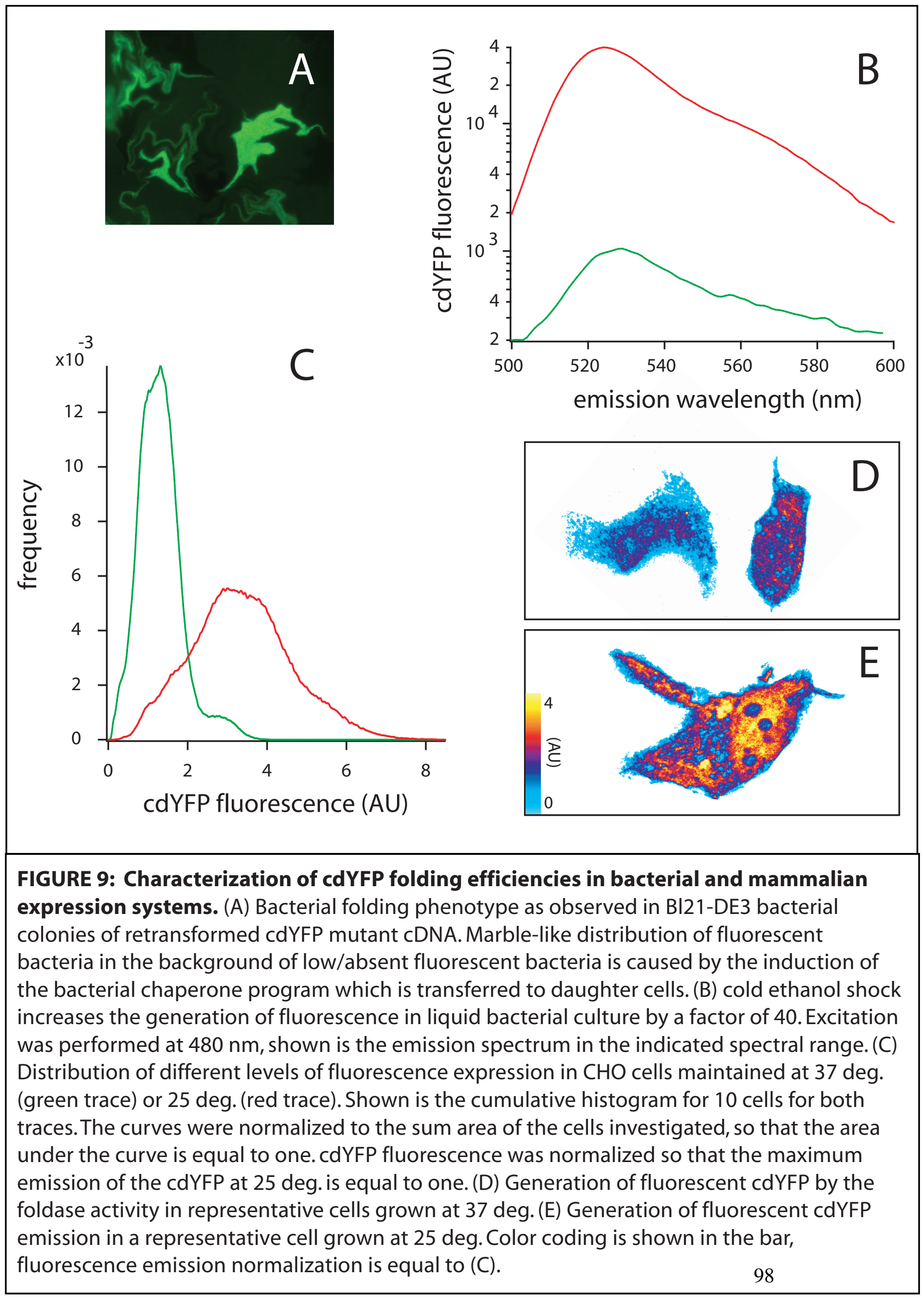


The increased dependence of cdYFP folding on chaperone activity was confirmed by the fact that the fluorescence yield was greatly increased by the induction of chaperones in liquid bacterial culture after cold ethanol shock (Thomas et al. 1996), which gave rise to an \pm 40 fold increase in fluorescence yield (Fig. 9B) and the appearance of a clear peak at the YFP chromophoric position $(514 \mathrm{~nm})$ in the absorption spectrum of the treated bacteria that was undetectable in the uninduced situation. The YFP mutant that showed the largest increase in fluorescence during this treatment was chosen for the construction of the folding sensor.

This mutant showed a 40 -fold increase in fluorescence and a clear peak at $514 \mathrm{~nm}$ in its absorption spectrum, indicative of efficient formation of the chromophore. Final fluorescence yields were comparable to those of unmutated EYFP, indicating that chaperone upregulation can rescue the loss of fluorescence due to folding impairment.

\subsubsection{Response of Mammalian cells for Foldase sensor under stress related conditions}

This folding mutant cDNA (cdYFP) was subcloned into a mammalian expression vector pEYFPN1 (Clontech), to allow expression in mammalian cells, Transient expression of this clone at 37 degrees in $\mathrm{CHO}$ cells showed a homogeneous distribution of low fluorescence intensities between different cells (Fig. 3C green trace, D). This shows that Overexpression of poorly folded cdYFP by itself is not sufficient to significantly up regulate mammalian chaperone activity. When expressed at low temperature (25 degrees), in order to relax folding constraints, a 3-4-fold 
increase in fluorescence was observed, which confirms the impaired folding pathway of cdYFP (Fig 9C, red trace, E).

\subsubsection{Structural characterisation of folding mutant}

Sequencing analysis of cdYFP clone (clone number 5 out of 17) selected for our study revealed a Glycine to Serine substitution at amino acid position 32 of YFP. This position is located near the end of the second beta-strand of the barrel. The 11 betastrand barrel surrounding the central alpha helix that contains the chromophore represents a difficult folding task. The correct conformation of the second beta-strand end and presumably the transition into the third beta-strand through only a small loop is apparently critical for correct formation of the entire barrel that shields the chromophore and provides the proper amino acid side-chain context for the chemical formation of the chromophore. Chaperone-mediated folding at this critical initial part of the YFP polypeptide corrects the gross structural defect in the barrel and allows the YFP structure to settle into a form that can accommodate fluorescence.

\section{Figure B: Mutation site of folding mutant}

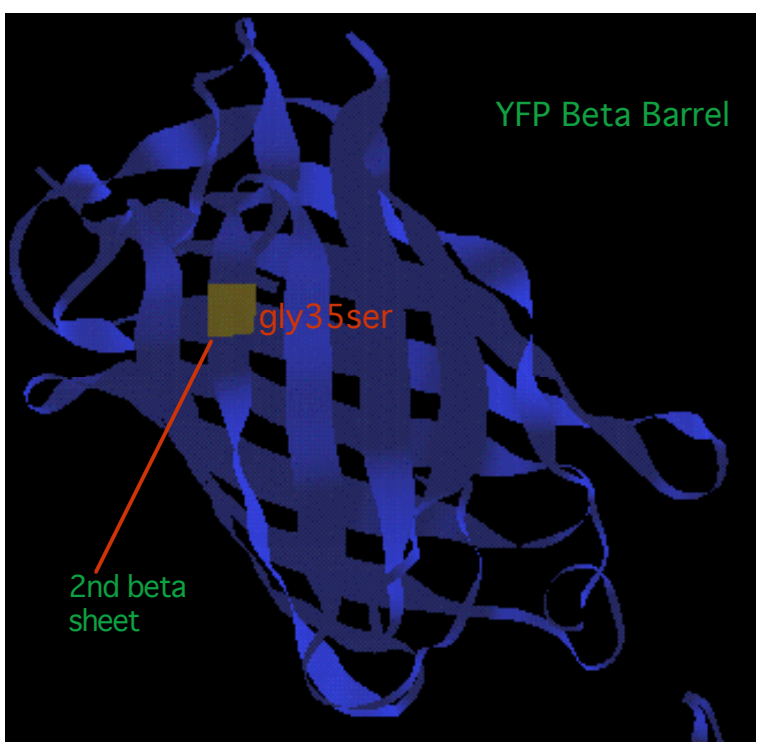

\section{Folding mutant of YFP:}

Yellow Fluorescent Protein used for folding sensor illustrated in a picture shows the amino acid substitution Gly35Ser (yellow) at the 2nd Beta sheet marked (Blue ribbon), which presumably incrases the folding constraint for the YFP molecule and is in need of chaperone assisted folding for the active chromophore formation inside the folded Beta barrel responsible for fluorescence emission and high quantum yield. 


\subsubsection{Measuring the folding efficiency of folding mutant in mammalian cells}

To be able to determine the concentration-independent folding efficiency of the cdYFP under the different experimental conditions, a concentration reference fluorophore was introduced. In one implementation, an HA antibody epitope was introduced at the cdYFP C-terminus, to yield HA-cdYFP. The total concentration of expressed fluorescent and non-fluorescent cdYFP can be determined from the fluorescence emission intensity of a Cy5 fluorescently labelled secondary antibody. This is used to obtain the distribution and relative concentration of folded cdYFP by image division. In an alternative implementation of the folding sensor, CFP can be coexpressed to serve as a reference fluorophore. The advantage of using CFP reference over antibody staining is that this assay can be used in living cells, however, at the cost of quantitation accuracy as the levels of translated CFP and cdYFP cannot be precisely controlled. We used the quasi-quantitative co-expression method of magnetassisted transfection and found essentially identical results for the measurements described below, but with higher noise content and cell-to-cell variation In case of the antibody reference, the reference is on the same molecule as the folding fluorophore, giving rise to robust and sensitive signals.

\subsubsection{Chaperone-mediated folding assay of foldase sensor in mammalian cells}

To show the sensitivity of the HA-cdYFP folding biosensor towards the major mammalian chaperone Hsp70, we compared the folding efficiencies of the sensor in wild type CHO cells with those obtained upon transient co-expression of CFP-Hsp70. In the wild type cells, a single prominent folding peak can be observed (Fig. 10A, 
Figure 10: cdYFP folding in mammalian cells is dependent on Hsp70.
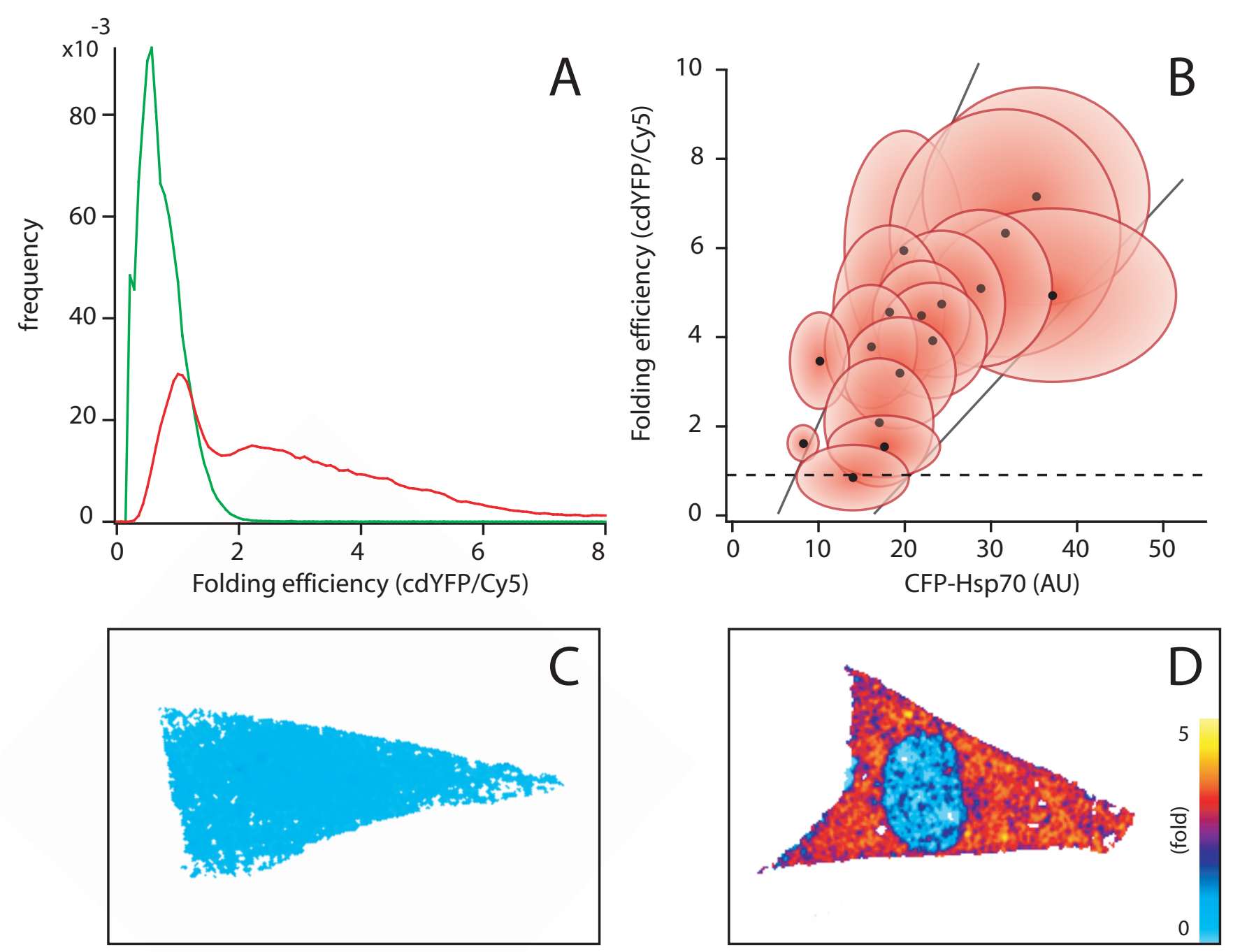

Figure 10: cdYFP folding in mammalian cells is dependent on Hsp70.

(A) Generation of fluorescent cdYFP in wild type $\mathrm{CHO}$ cells (green trace) and CHO cells transiently expressing Hsp70 (red trace). Shown is the cumulative histogram for 16 cells for both traces. The curves were normalized to the sum area of thecells investigated, so that the area under the curve is equal to one.cdYFP folding efficiency, as expressed by the ratio of cdYFP fluorescence and Cy5- anti HA immunofluorescence, was normalized to the position of the major folding peak representing partially folded cdYFP in wild type, untreated cells, as identified by comparison with the other experiments. This intermediate can be seen as separate peak in the red trace and high-end shoulder in the green trace at a folding efficiency of one. (B) Two-dimensional histogram of the distributions of folding efficiency versus the distributions of CFP-Hsp70 expression, as judged by the fluorescence emission of the conjugated CFP fluorophore, on a cell-by-cell basis for all 16 cells used to construct the red trace in (A). (C) cdYFP folding efficiency in a representative wild type $\mathrm{CHO}$ cell. (D) cdYFP folding efficiency in a representative cell expressing $\mathrm{Hsp} 70$. Color coding is shown in the bar, folding efficiency normalization is equal to (A). 
green trace C). Co-expression of $\mathrm{Hsp} 70$ in $\mathrm{CHO}$ cells shows a dramatic increase in the folding ratio (Fig. 10A, red trace, D) with a skewed distribution to very high ratios. The use of a CFP-labelled Hsp70 allowed us to relate the expression levels of the HSP to the folding efficiency on a cell-by-cell basis. The emission of the CFP-Hsp70 can be collected without interference from the higher wavelength fluorophores used in foldase sensor experiments (YFP and Cy5) and gives a information for the expression levels of the chaperone. The distributions of folding activity and expression levels were plotted against each other to reveal a clearly correlated relationship (Fig. 10B). This demonstrates that the foldase assay reports on the level of the Hsp70 chaperone foldase activity.

\subsubsection{Foldase sensor in sensing the activity HSP70 using BAG1 a co-chaperone expressing stable cell lines}

We then proceeded to compare the effect of stable overexpression of BAG1 and its Cterminal deletion mutant, BAG $\Delta \mathrm{C}$ in CSM14.1 Nigral cells (Fig. 11B). Wild-type CSM cells exhibit the same narrow distribution of relatively low cdYFP fluorescence, as the wild-type untreated $\mathrm{CHO}$ cells (Fig. 11A, green trace). overexpression of fulllength BAG1 protein dramatically changes this distribution to a new broad and high folding efficiency distribution being detected in these cells (Fig 11A, red trace). The majority of folded cdYFP exhibits \pm 2.5 fold increase in fluorescence, but the folding efficiency ranges to a 7-fold increase over the wild type situation. This shift is, in magnitude, comparable to the effect obtained with transient, high-level overexpression of Hsp70, implying a possible regulatory effect of BAG1 on Hsp70 and or other chaperones. 
This broad folding efficiency distribution is due to the presence of differently folded intermediates in the different analyzed cells. The insert in Fig. 11A shows that the BAG1-overexpression intensity distribution traces is composed of discrete intermediate distributions. These consist of species with \pm 2 -fold (blue curves), 3-4 fold (green curves) and 4-5 fold average increase in folding activity. The stably expressing $\mathrm{BAG} \Delta \mathrm{C}$ mutant, lacking Hsp70 binding capacity sites fails to produce this increased folding response in stably expressing CSM14.1 cells (Fig 11A, blue trace). This demonstrates the critical importance of the integrity of the BAG-binding domain for its chaperone upregulation activity. In fact, the folding efficiency distribution of the BAG $\Delta \mathrm{C}$ mutant shows a bi-modal distribution with a small peak overlapping with the position of untreated and wild-type cells (green traces in Figs. 9C, 10A, 11A) and a new, major peak at lower folding efficiencies $( \pm 0.8)$. We assign the recurring prominent peak in untreated and native cells to a stable folding intermediate towards properly folded cdYFP (which we normalize to 1). The new low-efficiency peak observed in the BAG $\Delta \mathrm{C}$ mutant, which is also present as shoulder in the untreated and native folding traces, represents the most poorly folded intermediate that can be assigned in cells. We assign folding ratios greater than 2 to more fully folded forms. These forms are only observed upon active induction of cellular folding activities, indicating that the endogenous chaperone activity apparently does not suffice to process the cdYFP beyond the first partial folding step.

The foldase activity needed for further folding obviously exceeds the natively present one. Upon induction of chaperone activity, both by Hsp70 overexpression or by modulation of endogenous Hsp70 by BAG1 overexpression, the folding pathway 
Figure 11: Foldase sensor characterisation in BAG1 expressing stable cell line

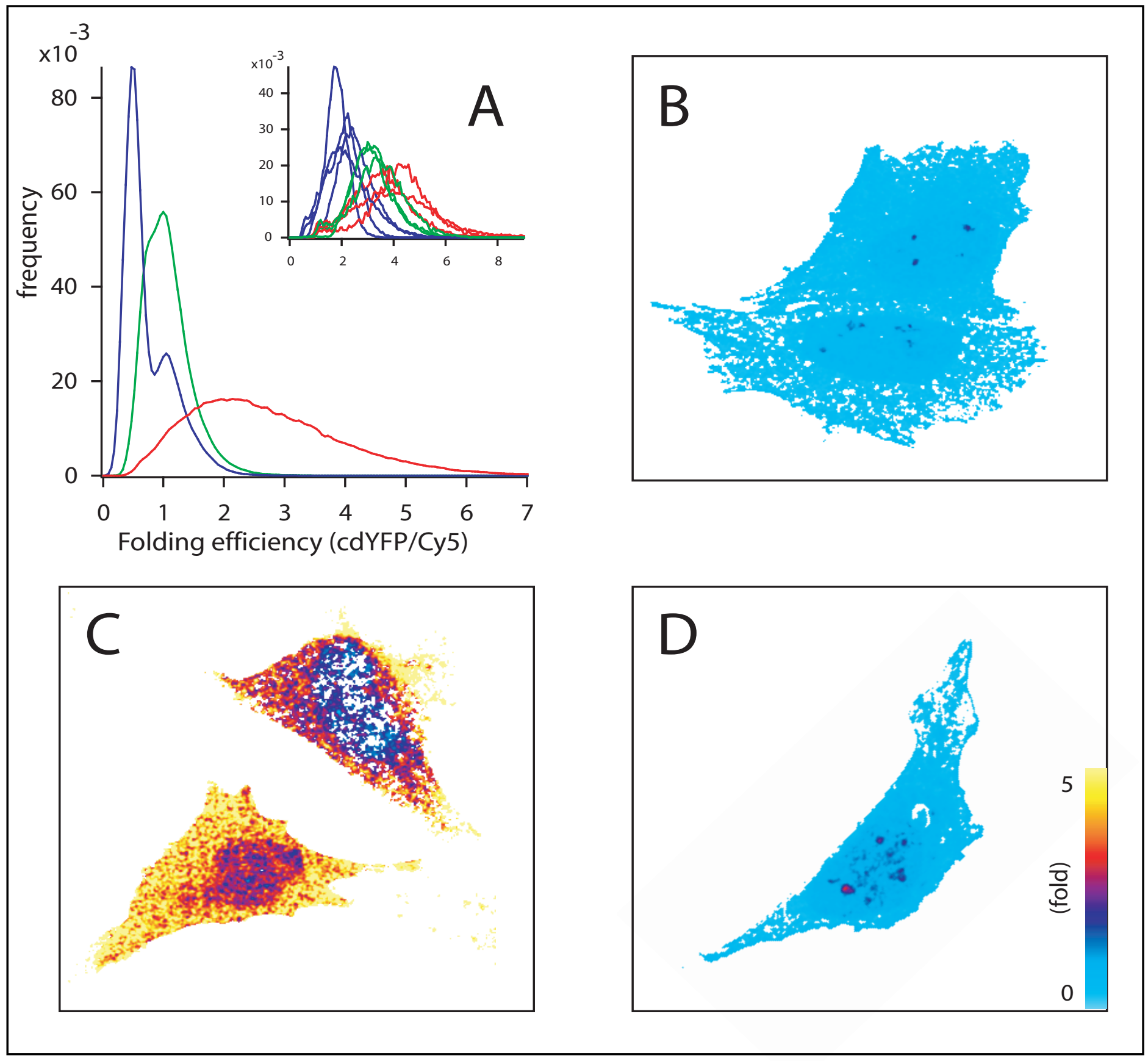

Figure 11: cdYFP folding sensor demonstrates the increase in chaperone activity by over expression of BAG1 in CSM cells. (A) Generation of fluorescent cdYFP in wild type CSM cells (green trace), CSM cells stably expressing BAG1 (red trace) and CSM cells stably expressing BAGdelC (red trace). Shown is the cumulative histogram for 10 cells for all traces. Normalization of folding efficiency and frequency are the same as in Figure 4. Insert shows the individual traces for the 10 cells that were used to construct the red trace. These traces were color coded to discriminate cells exhibiting lower folding (blue), intermediate folding (green) and high folding activity (red) in the population of stably BAG1 expressing CSM cells. (B) cdYFP folding efficiency in a representative wild type CSM cell. (C) CdYFP folding efficiency in a representative CSM cell stably expressing BAG1.(D)CdYFP folding efficiency in a representative CSM cell stably expressing BAGdelC. Color coding is shown in the bar, folding efficiency normalization is equal to (A). 
apparently diverges to allow graded "tuning" of folding forms giving rise to a broad folding efficiency spectrum with different forms representing low, intermediate and high folding efficiencies. Only $\pm 25 \%$ of cdYFP is partially folded in BAG $\Delta \mathrm{C}$ expressing cells, in comparison to $>90 \%$ for the wild type cells. This implies that truncation of the HSP70 binding domain of BAG generates a strong dominantnegative phenotype with respect to folding since folding activity is almost completely impaired.

In our experimental paradigm, the wildtype CSM-cells, as well as CHO-cells show a single prominent peak of folding activity. By comparison with the different treatments, this peak could be assigned to a stable folding intermediate. The experiments with the BAGDC cells showed the presence of a further folding intermediate of lower efficiency. In retrospect, the lower efficiency shoulders of the folding efficiency distributions of wild type and untreated cells could be seen to contain a fraction of this poorly folded "base" condition of the sensor (Fig. 11A green versus red trace and Fig. 11A). Wild type cells are not able to overcome the 'second' step in protein refolding. In contrast, cells stably overexpressing BAG1 or Hsp70 directly show a prominent high-efficiency distribution indicating further advanced protein refolding. This distribution is broader and less symmetrical as the poorly and initially folded distributions.

These high-folding activity distributions, when compared on a cell-by-cell basis, demonstrate the progressive dependence on the folding threshold on Hsp70 expression levels (Fig 10B) and on discrete 'fine tuning' steps at more advanced refolding activities, e.g. upon BAG1 overexpression (Fig. 11A insert). Surprisingly 
BAGDC, which is lacking Hsp70 binding C-terminus, does not yield similar results to wild type cells, but seems to even further impair protein refolding. This observation is in line with results by others, where the influence of BAG1 and a C-terminal deletion mutant of BAG1 on HSP70 ADP turnover were measured. They found a \pm 900 -fold increase in ADP turnover in cells transfected with BAG1, whereas cells transfected with the deletion mutant displayed a four-fold decrease (Gässler et al. 2001). This effect was explained by the presence of an additional domain situated N-terminally of the BAG domain which is apparently important for binding and activation of chaperones and which acts in a dominant negative manner on chaperone foldase activity.

\subsection{Analysis of growth cone protein transport, degradation, synthesis and folding in neurons and neuron-like PC12 cells}

In the following section I describe how we analysed the local protein turnover machinery of growth cones in neurons and in neuron like cells PC12 cells, using the above mentioned biosensors for transport, synthesis, degradation and folding of protein, We primarily used the PC12 cell line to investigate the aspect local machineries, because they are considered to be good model system to study neuronal processes, which simulate the neuron like properties and produce neuron like processes, upon NGF treatment (Greene LA et al 1976 and Tischler AS et al 1978). PC12 cells treated with NGF and differentiated for 5 to 9 days for complete differentiation. These differentiated cells were transfected with the respective 
constructs for the ubiquitination, protein synthesis and HSP70 induced protein folding, to asess their relevance in growth cone local protein turnover.

\subsubsection{Analysis on protein transport machinery in neuronal growth cone.}

\subsubsection{Synaptophysin p38 tagged FLAP Sensor in transport assay}

We chose synaptophysin p38 as the transport marker, since it is well characterised (Kaether $\mathrm{C}$ et al 2000) in hippocampal neurons as axonal transport marker. For this construct we used cDNA of rat synaptophysin p38, C-terminally tagged with FLAP CFP-YFP tag. The construct was subcloned into the neuron specific synapsin promoter to allow neuron specific expression of tagged protein. Constructs were transfected and cells were grown for 7 days until there is extended axonal growth. FRAP experiments were performed with synaptophysin p38 FLAP tag as described above (see principle of FLAP tag in biosensor for transport) The experiment with synaptophysin p38 by FRAP analysis, we observed interesting features of transport. We observed synaptophysin in proto vesicles along the axon and synaptic vesicles of the presynaptic compartment. Further we calculated the net directionality of synaptophysin FLAP tag as bidirectional and Synaptophysin p38 was primarily distributed along the axon and in distal regions of axon, representing growth cone branching point (Figure 12A). Therefore, a net anterograde transport of newly synthesized protein would be expected, which is observed in our system of about $60 \%$ peak intensity (Figure12B) indicated by a peak (tall red peak in Figure12B). 
Figure12: FLAP tag based FRET assay on axonal transport-Using synaptophysin p38
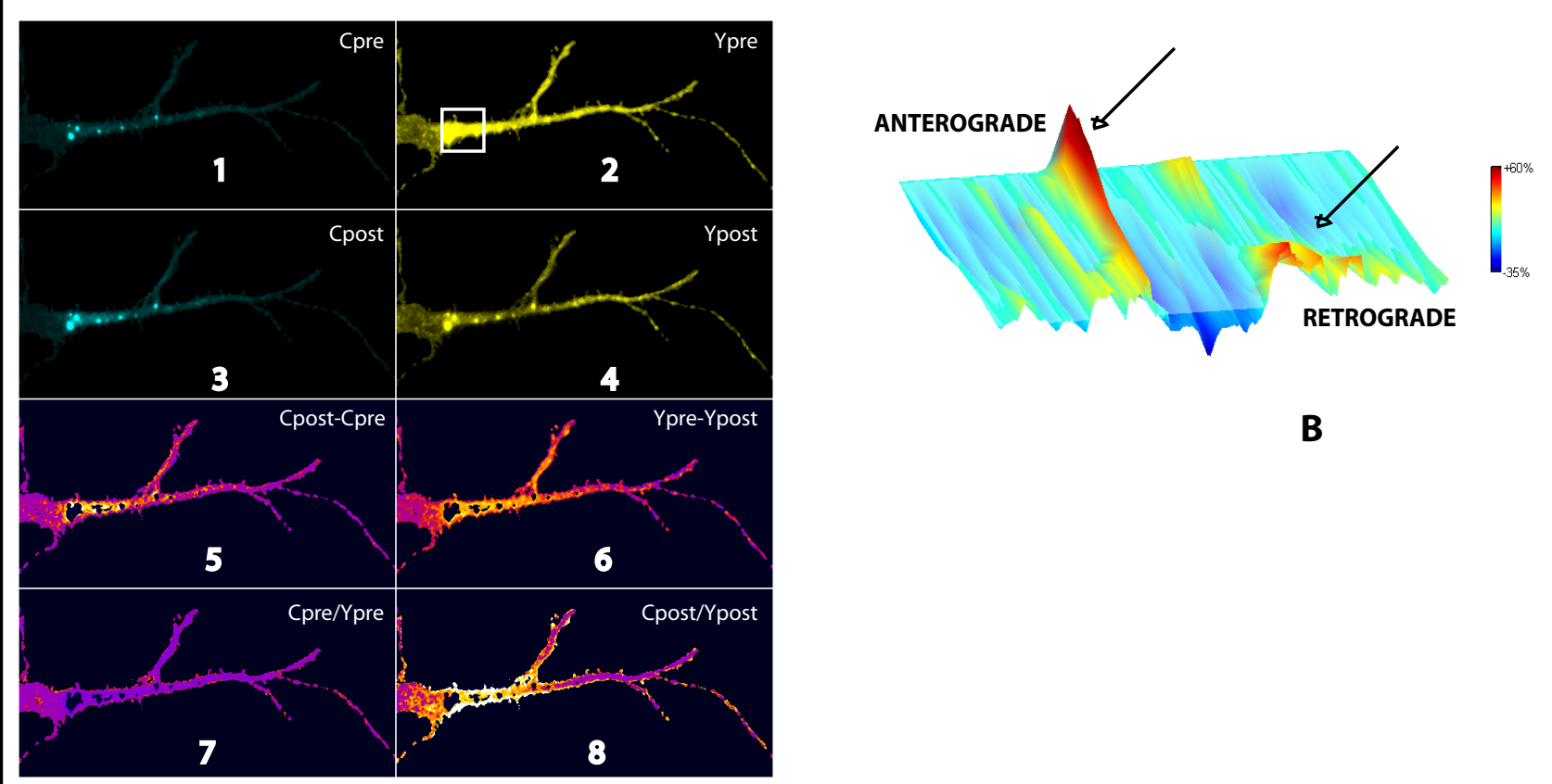

B

FRAP assay on FLAP TAG P38 Synaptophysin

Distribution analysis on FRAP image

\section{Figure 12: FLAP tag based FRET assay on axonal transport-Using synaptophysin p38:}

(A) Figure representing the steps of FRAP analysis protocol using synaptophysin P38 tagged FLAP microscopy. Upon exciting CFP we see both CFP and YFP in the (FigureA, panel1,2) indicates FRET. By bleaching YFP fluorescence on the marked region(Box panel2) we see the recovery of CFP(FigureA panel3,4). On creating the mask of CFP post bleaching with YFP post bleaching gives the information of local change in fluorescence ratio as it is give for before bleaching also (FigureA panel 5,6). By calculating the ratio of CFP quenched due to FRET over and CFP after recovery gives the ratio of protein flow in the axon due to the mixing of fractions of CFP quenched over recovered (FigureA panel 7,8). (B) Distribution analysis of protein fraction in FRAP analysis with the peak intensity. Picture represents the directionality (anterograde to retrograde) and peak intensity of protein in motion at any particular direction (Red peak represents maximun intensity and the the decrease in ratio flow represented by yellow to blue in (FigureB lookup table). Net anterograde movement of synaptophysin p38 FLAP tag calcuated from CFPpost and YFP post bleaching is $65 \%$ and net retrograde was counted as $35 \%$, see wave front of motion in (Figure B) indicated by arrow. 
The presence of predominant net anterograde measured in our system is not surprising, since the synaptic vesicles (predominantly tagged with synaptophysin p38) target the presynaptic vesicles primarily to the distal end of axons (Nakata et al., 1998; Prekeris et al., 1999). Therefore switching the fluorescence emission in FLAP tag synaptophysin from YFP to CFP spectrum upon bleaching, presumably converts the population of tag at a given time along the axon (Figure12A CFP ${ }^{\text {post }} / \mathrm{YFP}^{\text {post }}$ ) when compared to $\mathrm{CFP}^{\text {post }} / \mathrm{CFP}^{\text {pre }}$. This experimental stratergy allow us to determine the direction, wave front of movement, peak intensity of gradient and steepness in the gradient of presynaptic deposition (Figure 12B, red peak in distribution durve ). It might as well be speculated that, population labelling in the presynaptic compartment could provide valuable information on synaptic vesicle deposition and protein trunover phenomenon.

\subsubsection{Analysis on protein degradation machinery in the growth cone.}

\subsubsection{Ubiquitination of growth cone like structures in PC12 cells}

The transfected cells were checked for the localisation, activation and interaction of proteasome complex by lifetime based FRET and ratiometric FqRET analysis. For these experiments we used the GFP fused with PEST efficient ubiquitin signal sequence, which acts as a target sequence for proteasome-mediated degradation and REACh2 labelled Ubiquitin as a constitutive ubiquitination signal. Lifetime measurement was performed on GFP-PEST as donor flurophore and REACh-Ubq as a dark acceptor (ubiquitination assay). The results were found to be similar in terms of ubiquitination and antibody localisation to the finding we obtain from the 
Figure 12: Protein ubiquitination assay in Neuron like PC12 cells

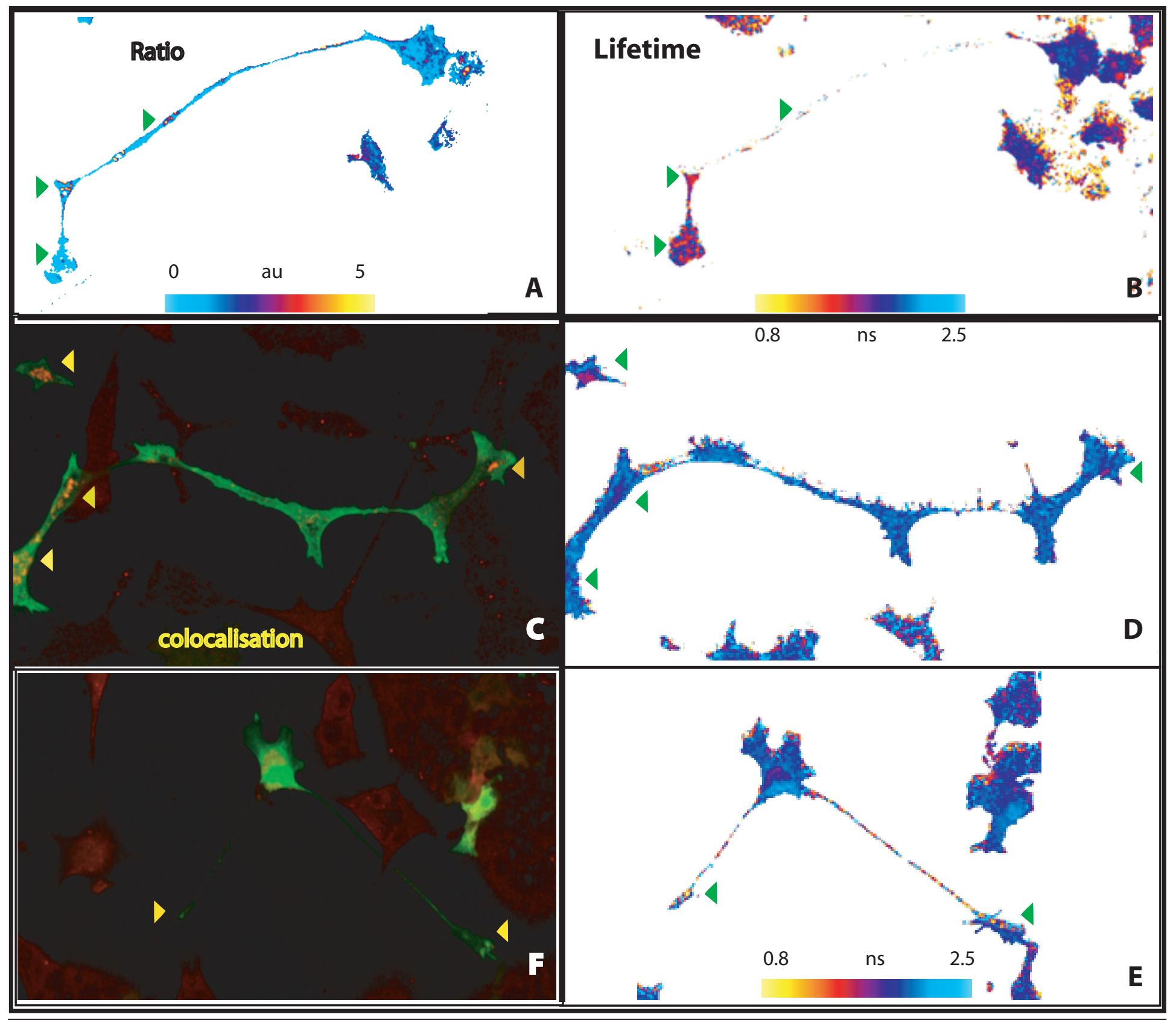

Figure 12: Protein ubiquitination assay in Neuron like PC12 cells

(A) FqRET image of PC12 cells coexpressing GFP-PEST AND REACh2-ubiquitin shows an increased ratio at punctuates near the axonal branches and in the growth cone like structures (fig A green arrowhead). (B) FLIM image of the corresponding cell using TC-SPC 2p microscopy indicates the punctate structures at the same places with reduced lifetime. (C) PC12 cells showing antibody localization of REACh2 ubiquitin for Ubiquitin antibody in the micro clusters (yellow arrowhead). (D) Lifetime image of the corresponding antibody localization image, green arrowhead shows the complete matching between Ubiquitin aggregates and reduced lifetime (hot colours indicates high ratio and lower lifetime). (E) PC12 cells expressing PEST-GFP with REACh-ubiquitin, shows micro aggregates at the growth cone like structures with reduced lifetime(green arrowhead) matches at the pointed yellow arrowhead for antibody localization using proteasome specific antibody in (figure F) 
ubiquitination assay (see also results in section 4.1.2.6). The growth cones of PC12 cells as measured by FLIM, shows an active ubiquitination machinery with a lower lifetime and higher ratio FqRET (Figure 13A and B). The pointed arrow indicates the axonal branching point and growth cone like structures represents lower lifetime and higher ratio (Figure $13 \mathrm{~A}$ and $\mathrm{B}$ warm colour in lifetime and ratio). To verify that the structures we see are actually an active ubiquitin targetted proteasome complex we used antiobody based immuno localisation and lifetime measurement(ubiquitination assay section 4.1.2.6).

The localisation with anti ubiquitin antibody shows striking similarities to the structures representing the reduced lifetime and the active ubiquitination machinery was mostly in the axonal branches and nerve endings of differentiated PC12 cells see pointed arrow in ( Figure 13C and D). Reduced lifetime in untransfected cells which may be due to the sytem error or instrumental noice, can be elliminated by extended photon counting or by stringent masking. Our attempt to visualise the localisation of the proteasome complex in the active growth cones using antibody against $26 \mathrm{~s}$ subunit of active proteasome complex, failed to show any significant match to the structures corresponds to lower lifetime (Figure 13E and F). It might be due to the intrinsic proteasome machinery which is formed but is not active or inturn are not completely formed and interacting with the destabilised proteins (Figure 12E and F). Arrows in Figure $\mathrm{E}$ and $\mathrm{F}$ indicates the regions of reduced lifetime with no significant increase in the ratio of GFP/CY5 of FqRET analysis (see materials and methods for FqRET method). 


\subsubsection{Growth cone of $\mathrm{PC} 12$ cells show decrease in protein content}

In our experiments with NFM-GFP (Neurofilament-M) constructs transfected in PC12 cells, we noticed the presence of protein degradation machinery in the growth cone like structures. In this experiments we used differentiated PC12 cells, transfected with NFM-GFP in the mammalian expression vector driven by the $C M V$ promotor. Confocal based Fluorescence Recovery After Photobleaching (FRAP) analyses were performed on living cells, where in cells expressing GFP proteins in growth cone like structures were bleached with $100 \%$ laser dose using Argon ion laser at $488 \mathrm{~nm}$. The bleached region was followed within 30 min with $30 \mathrm{sec}$ intervals under temperature and buffer controlled conditions. The transport of protein from the cell soma was blocked using two inhibitors, namely

A. Nocodazole, which non-specifically inhibit both anterograde and retrograde transport of molecules toward and away from growth cone like structures due its microtubule depolymerising properties.

B. Taxol, which is specific for promoting microtubule destabilising properties and inhibiting anterograde transport to the distal end of nerve.

Differentiated PC12 cells were transfected with NFM-GFP cDNA and cultured for 18 hours in-vitro. The protein transport was blocked by adding $100 \mathrm{ng} / \mathrm{ml}$ nocodazole and 50ng/ml of taxol for $15 \mathrm{~min}$ before performing the FRAP experiments. In Comparison with control experiments (Figures 14 picture 1,2,3, Graph 1,2,3) which is not treated with any inhibitors, the growth cone like structures of samples treated with Nocodazole that demonstrating collapsed morphology shows a decrease in total 


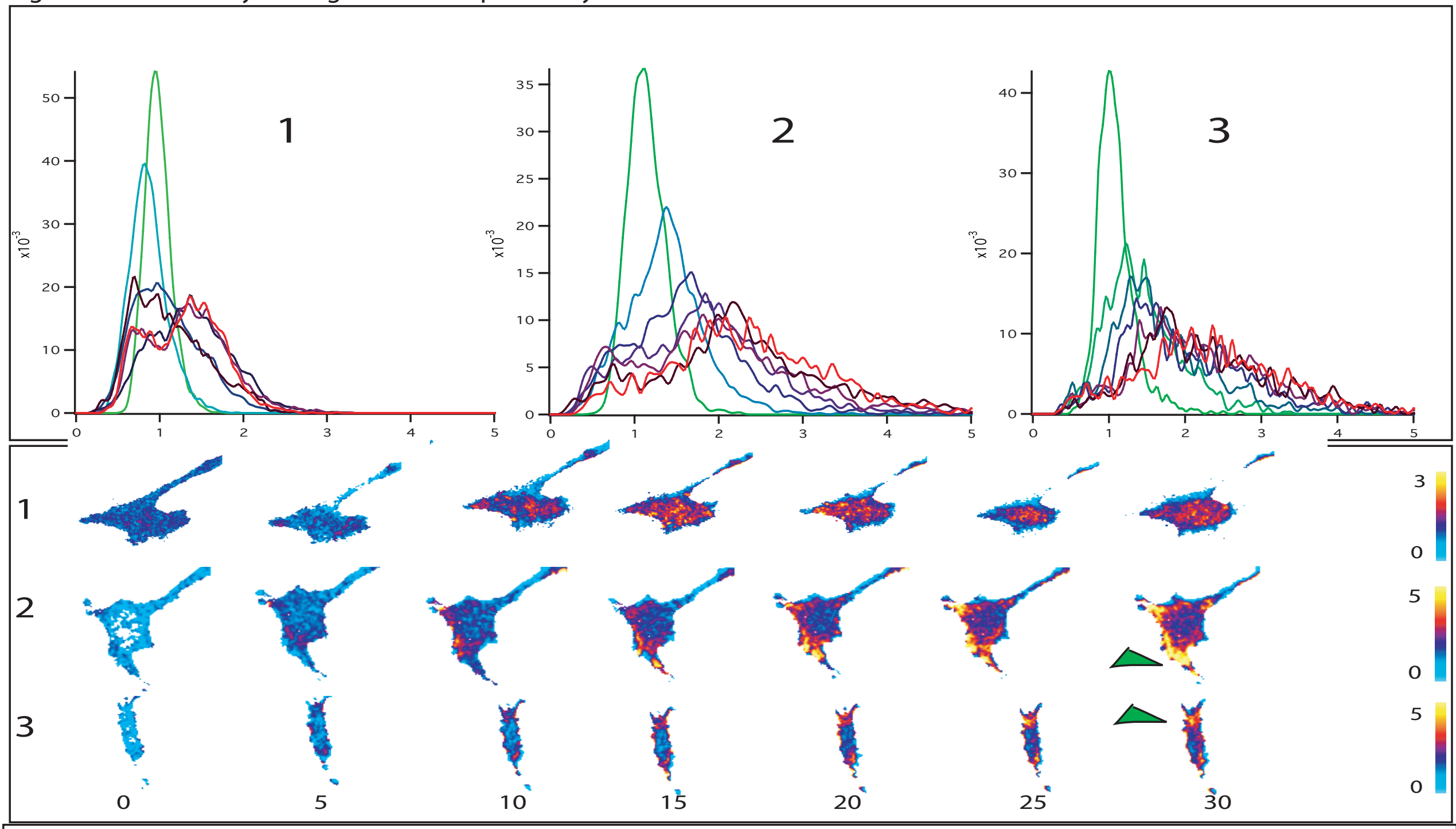

Figure 14: Growth cone like structures of PC12 cells synthesize protein - FRAP analysis. $(1,2,3)$ Graph and pictures of growth cone like structures in PC12 cells showing protein synthesis in short duration of time ranges from 0-30 min at 5 min interval.FRAP analysis on growth cone protein synthesis shows growing growth cone like structures have local protein synthetic machinery and also indicate an increase in protein content at polarized regions of growth cone showing protrusion (Pointed arrowhead pic2,3). Graph $(1,2,3)$ shows relative frequency distribution and fold increase of protein content in the growth cone at each time point from 0 to 30 at 5 min interval (Green-0min, Blue-5min, Brown-10min, Pink-15min, Violet-20min, Black-25min, Red-30min).Protein content increases upto 5 fold after 30 min when compared to the control level at $0 \mathrm{~min}$, can be seen from hot colors in the lookup table (LUT) 


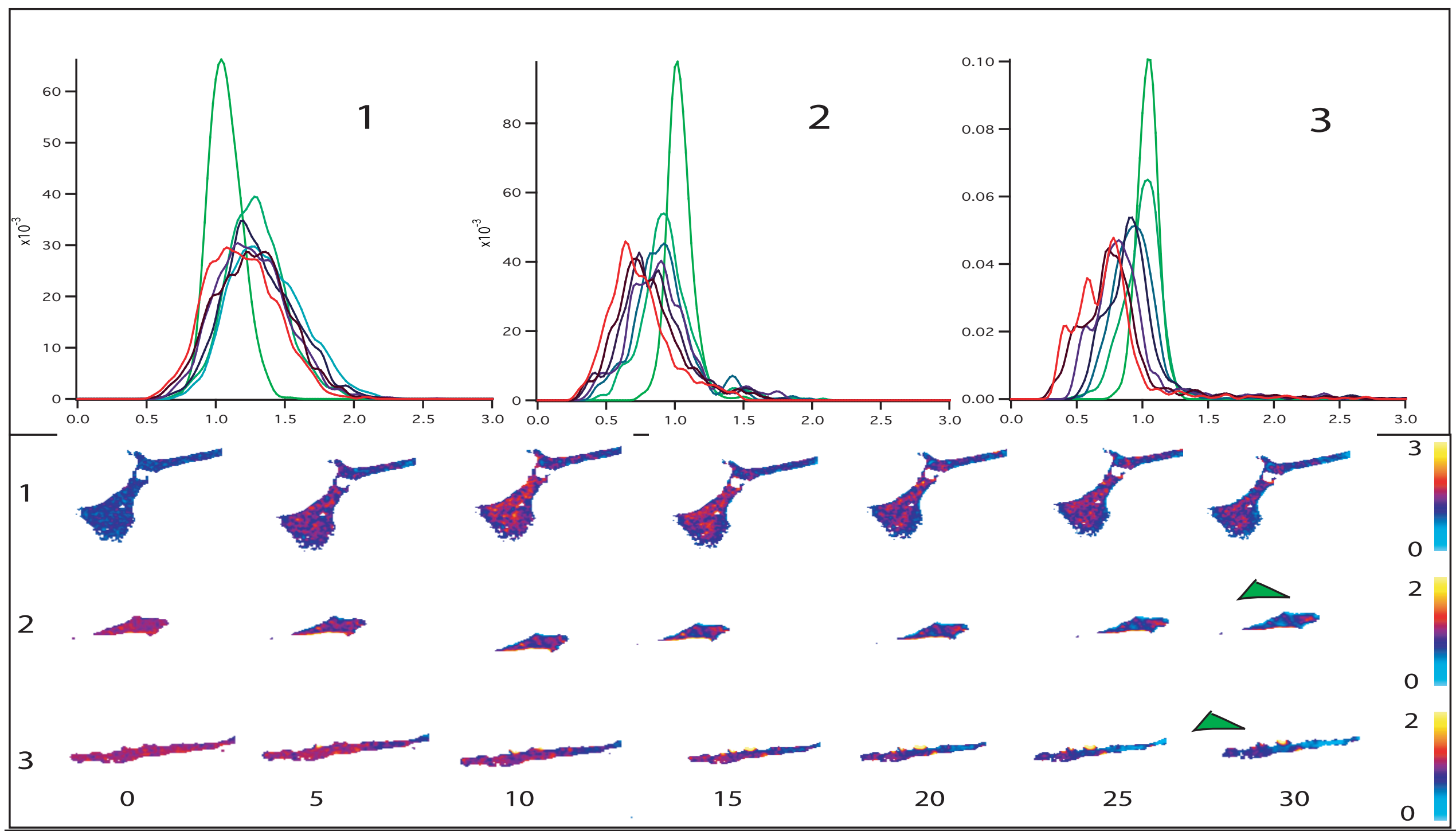

Figure 15: Growth cone structures of PC1 2 cells after Nocodazole treatement shows translation and degradation machinery

$(1,2,3)$ Graph and pictures of growth cone like structures in PC12 cells showing protein synthesis and degradation in short duration of time ranges from 0-30 $\mathrm{min}$ at $5 \mathrm{~min}$ interval.FRAP analysis on growth cone protein synthesis shows growing growth cone like structures have translation and degradation machinery and also indicate an decrease in protein content at coallapsing growth cone (Pointed arrowhead pic2,3). Graph 2,3) shows relative frequency distribution and fold decrease and (pic 1, Graph 1) fold decrease in protein content in the growth cone at each time point from 0 to 30 at 5 min interval. (Green-0min, Blue-5min, Brown-10min, Pink-15min, Violet-20min, Black-25min, Red-30min). Fold increase or decrease of protein content is represented in pseudo colour mafa LUT. 


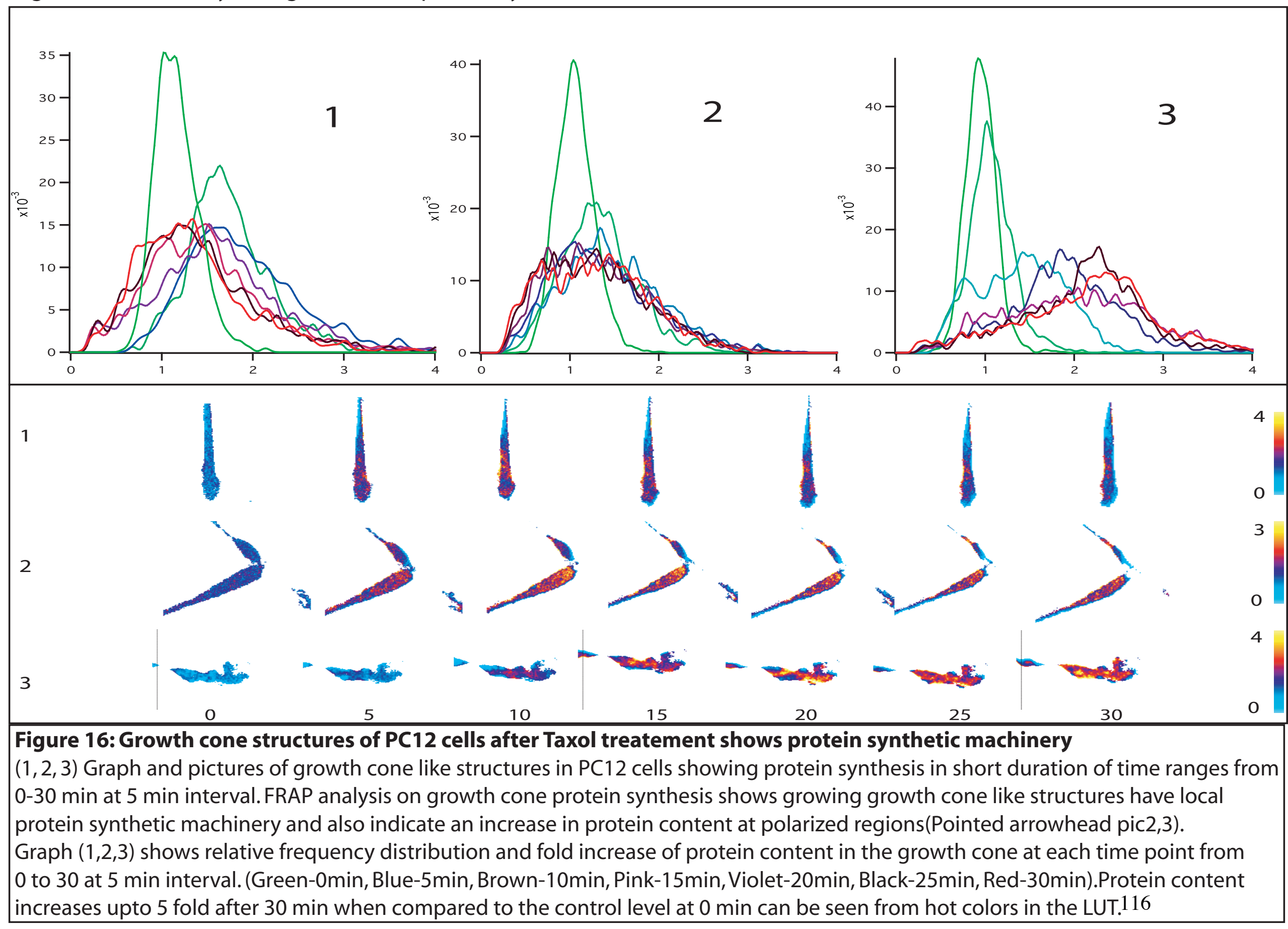


protein content up-to 2-fold (Figure 15 picture 2,3 and graph 2,3) also was observed in taxol treated cells at some localized spots (Figure 16 picture 1, Graph1) and also can be seen from distribution at $30 \mathrm{~min}$ (red trace in graph 1 Figure 16). The relative distribution of protein content is plotted in frequency distribution graph of ratio images at each time point ranges from 0-30 min with 5 min interval. From the results we obtain, it is evident that the collapsing growth cone tends to maintain the internal protein content by locally degrading them in a relatively short time frame. The extent in which protein degraded in growth cone is purely dependent on the activity of growth cone and the total pool of unused (toxic) proteins. To our knowledge, this is the first indication that NFM proteins are selectively degraded in collapsing growth cone like structures.

\subsubsection{Growth cones of Cortical Neurons show chemotropic response to protease and proteasome Inhibitors}

Protein turnover hypothesis was validated with the behavioral response of neurons under the effect of different stimuli and inhibitors. To show the inherent properties of the growth cone to change its the morphology upon applying different guidance cue, the reversibility of guidance in the presence and absence of inhibitors. We used Lyso Phosphatidic Acid (LPA) as guidance molecule and Lactacystin, Protease Inhibitor Cocktail (PIC) as inhibitor system. To study the chemotropic response of growth cone, we used embryonic cortical neurons in dissociated primary cultures as the model system. The growth cone collapse assay was performed with LPA, which is known to collapse many neuronal populations and was not tested in cortical neurons. 
As a positive control we used sema3a and Netrin1, which are known (campbell. DS et al 2001) for their collapse and repulsion in cortical neuron system.

\subsection{Collapse assay}

To perform the collapse assay we used cortical neurons from the embryonic mouse E18 which was seaded a day before treatment with LPA. Culture dishes were coated with PLO and no laminin, since laminin is known to inhibit the collapse of growth cone. Compared to control neurons at 18 hours after growth in culture, about $80 \%$ of the cortical neuron show collapsed growth cone morphology, (Figure 17) under the effect of treatment with $100 \mathrm{um} / \mathrm{ml}$ LPA for $15 \mathrm{~min}$. In order to study the effect of the proteasome and the protease in the LPA induced growth cone collapse, cells were additionally treated with lactacystin $(100 \mathrm{um} / \mathrm{ml})$ and PIC $(1 \mathrm{x} / \mathrm{ml})$, before adding the LPA.

We analysed several parameters for the the growth cone like morphology, neurite length and number of collapsed and intact growth cones. The analysis showed that growth cone collapse massively for LPA $(60 \%)$ and the was inhibited partially by lacatacystin (30\%) and PIC (50\%), while PIC treatment show a extended neurite length when compared to lactacystin (plot D, Figure17 and18). We further compared the effect of other guidance cues Sema3a (Figure 19) and Netrin1(Figure 20) on growth cone morphological behaviour. The results indicates that sema3a induced collapse $(50 \%)$ was inhibited by proteasome inhibitor lacatacystin (40\%) and PIC (50\%), but the Netrin 1 mediated growth or repulsion does not show any significant difference with inhibitors. (Plot E of Figure 20 and pic A,B,C,D). 
Figure 17: Effect of protease and proteasome inhibitors on LPA induced collapse

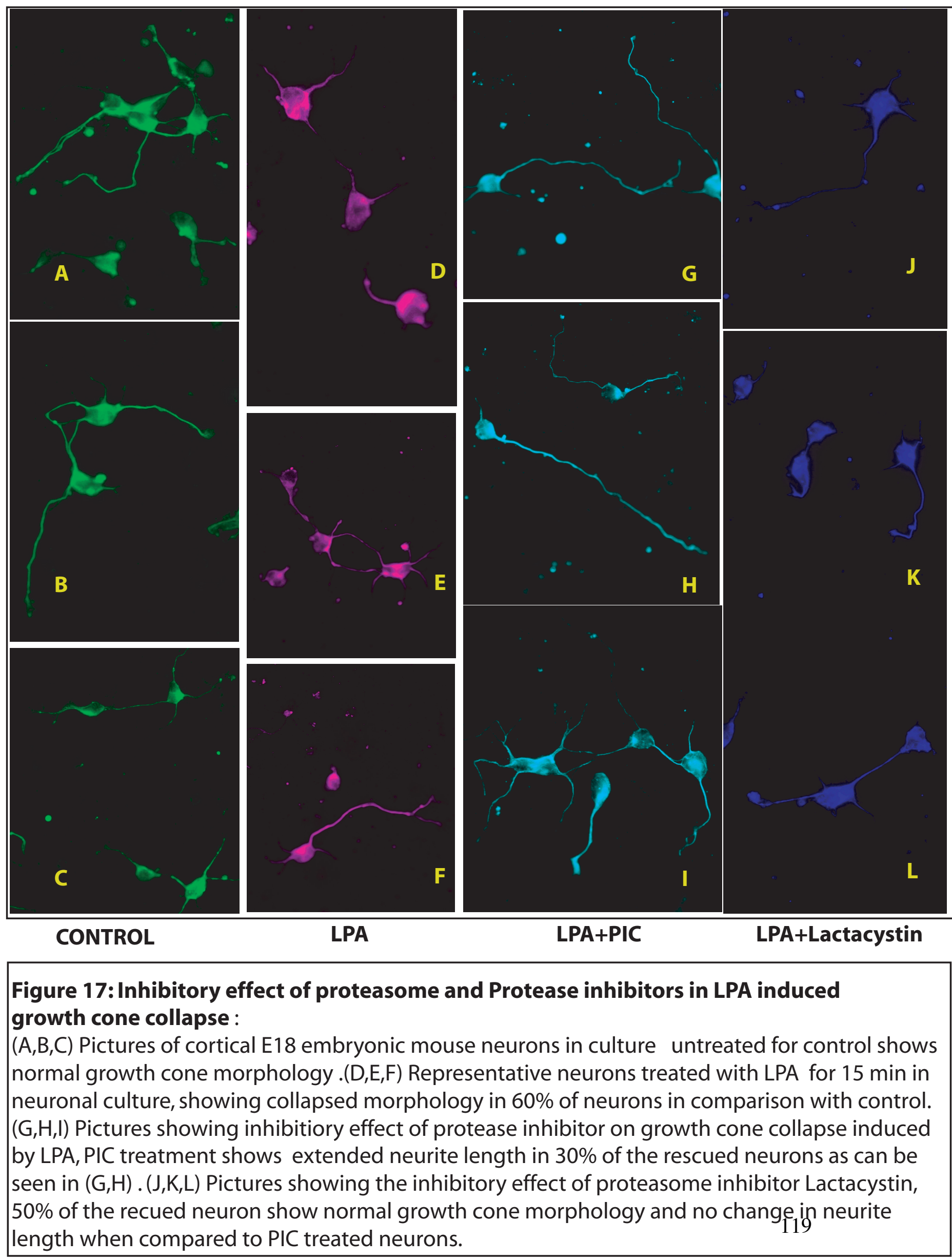


Figure 18: LPA induced collapse and the effect of inhibitors on growth cone collapse

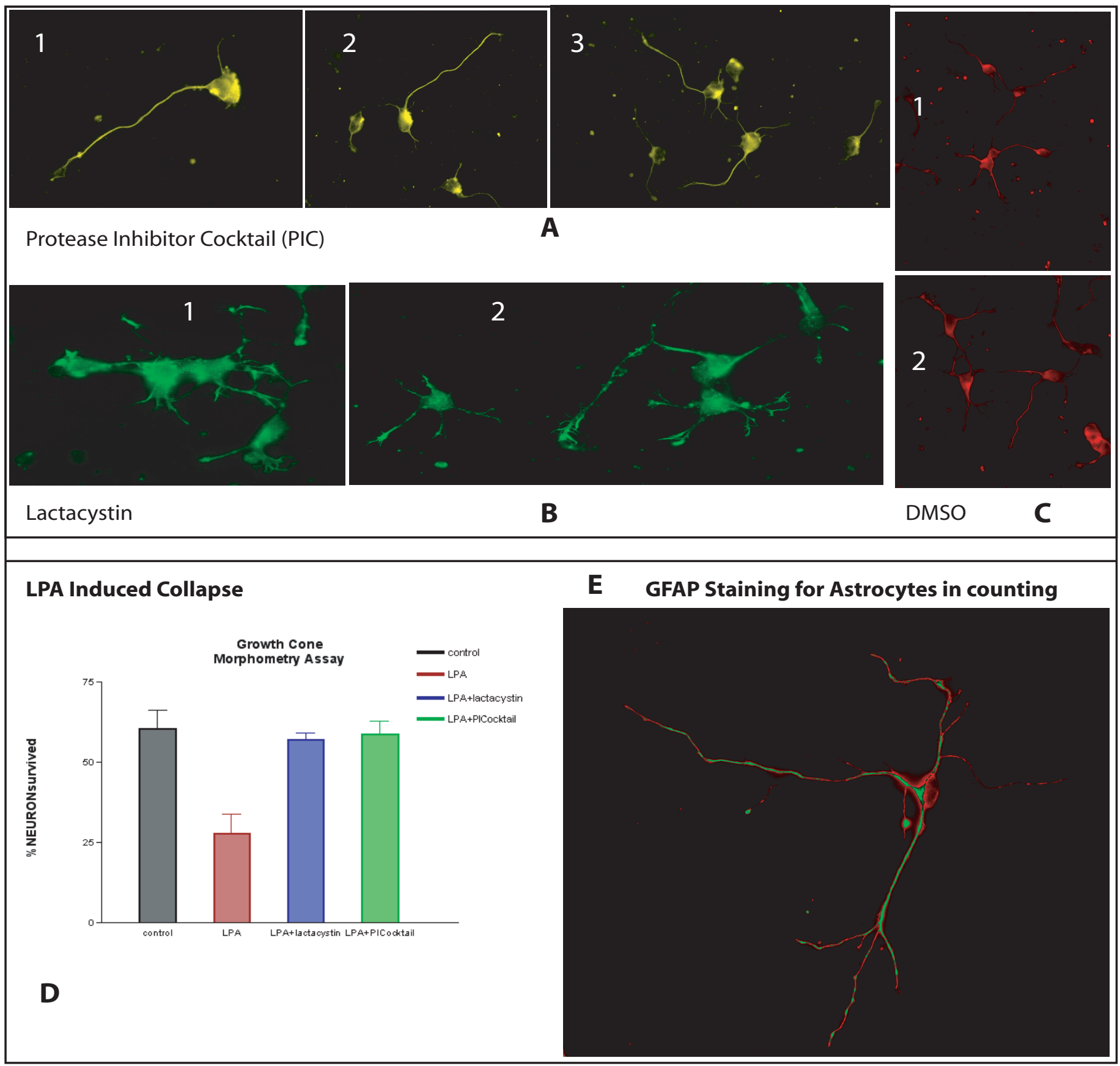

Figure 18: Inhibitory effect of LPAinduced growth cone collapse by proteasome and protease inhibitors

(A) Pictures of neurons representing control experiments on the effect of Protease (PIC) and proteasome (Lactacystin) inhibitors and in maitaning growth cone morphology with extended neurite length $(A, 1,2)$. (B) Lactacystin treated control neuron showing fine growth cone morphology with extensive branching $(B, 1,2)$ when compared to PIC treated neurons. (C) Control sample neurons showing no induced effect of collapse to DMSO treatment $(C 1,2)$,which was used to dissolve inhibitors of proteasome and LPA.

(D) Histogram demonstrating the percentage of neurons with normal growth cone morphology under differeent stimulus. (E) Control staining for Astrocyte specific antibody Glial Fibrillary Acidic Protein (GFAP), which was excluded in the counting for growth cone morphometry to correct for the neuronal populations in total. 
Figure 19: Effect of Inhibitors for proteasome and protease on Sema3a induced collapse

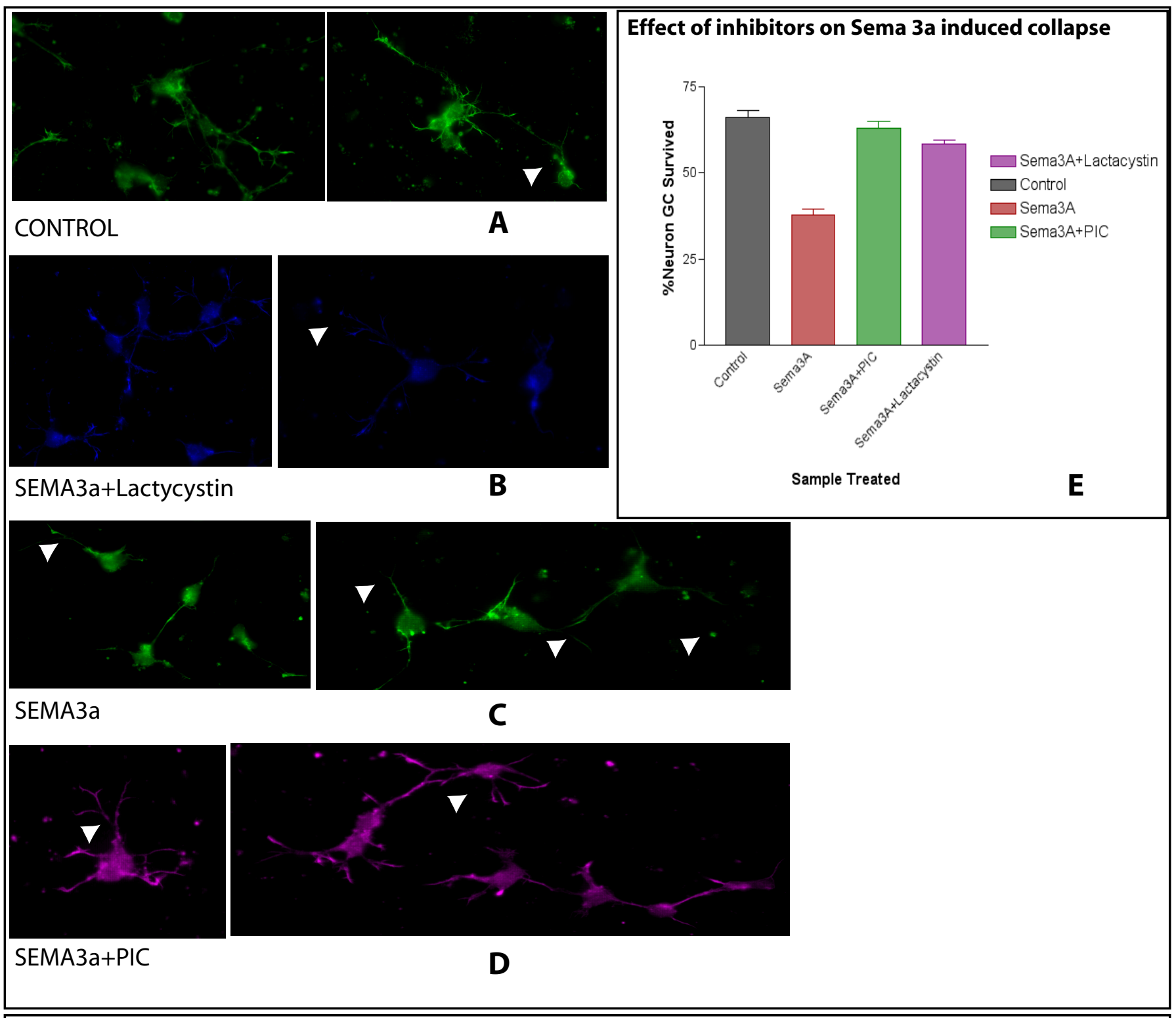

Figure 19: Effect of Inhibitors for proteasome and protease on Sema3a induced collapse (A) Untreated control neurons showing Normal growth cone morphology $(A, 1,2)$. (B) Inhibitory effect of lactacystin on sema induced growth cone collapse fig $(B, 1,2)$ shows rescued morphology when compared to the neurons collapsed upon sema 3a treatment $(C, 1,2)$ $(C 1,2)$ Neuronal pictures showing collapse induced by sema 3a after 15 min of treatment. $(D, 1,2)$ images of Neuronal population treated with PIC 15 min before adding Sema 3a shows a complete reversal of collapse induced by sema $3 a$ and most of the neurons upon PIC treatment shows extensive axonal branching and prominent growth cone. (E) Cummulative histogram showing the relative $\%$ of normal neuronal growth cone upon treatment with Sema $3 a$ and the relative inhibitory effect of protease and proteasome inhibitors on sema $3 a$ induced collapse (Arrow head shows the growth cone morphology upon different treatment) 
Figure 20: Effect of Inhibitors on Netrin1 induced growth or repulsion

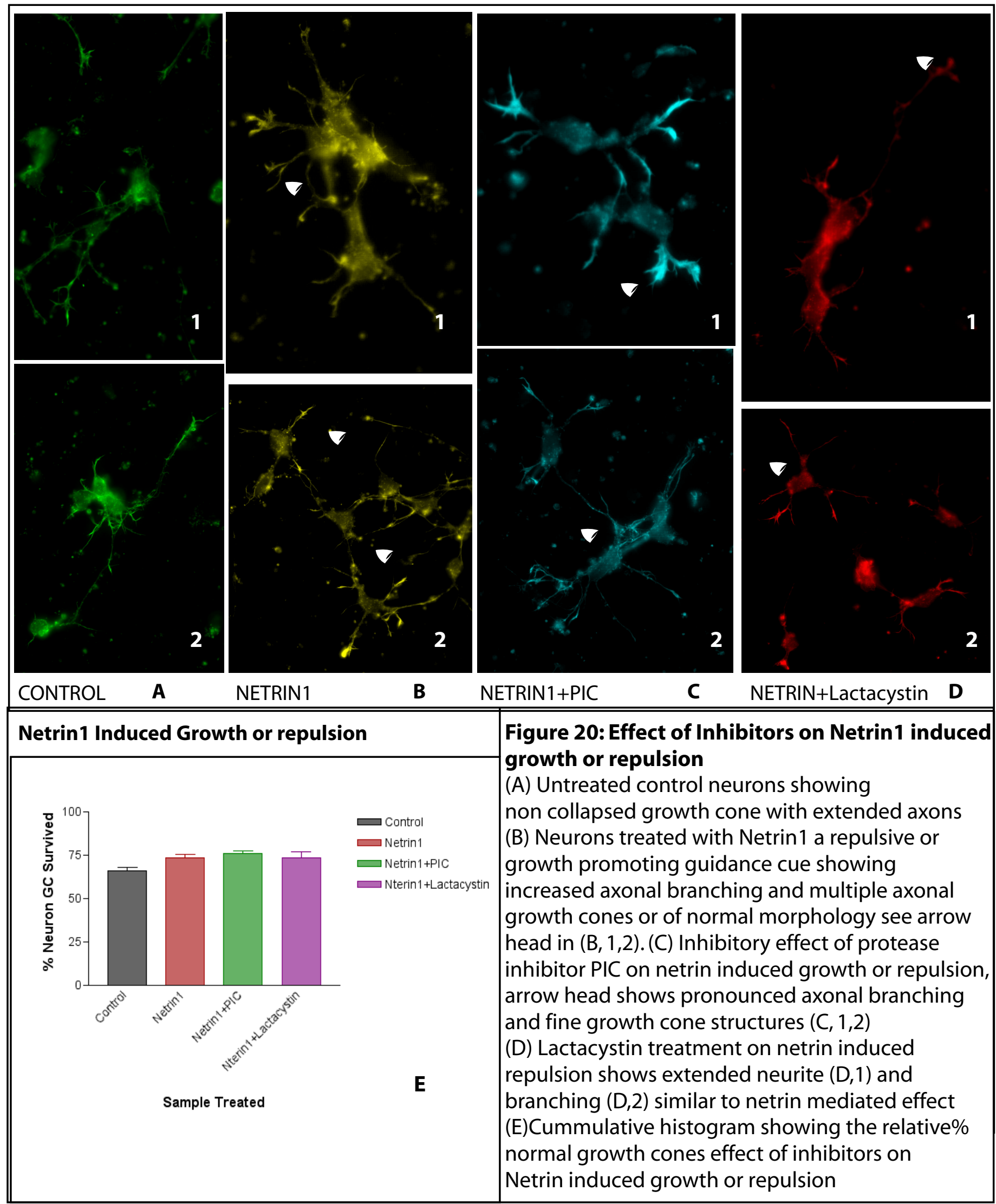




\subsubsection{Analysis on protein synthetic machinery in the growth cone}

\subsubsection{Growth cone like structures of $\mathrm{PC} 12$ cells has protein synthetic machinery}

Growing and extended growth cone like structures of PC12 cells have proteins synthetic machinery. From the FRAP experiments we perfomed on PC12 cells with and without transport inhibitors (Nocodazole and Taxol) showed a concluding observations that the growing structures at the tip of the PC12 cells demostrating the growth cone like morphology (Figure 14 pic 1,2,3, Figure 15 pic 1 and Figure 15 pic 3 ) synthesize proteins locally and the net synthesis ranges from 2 to 4 fold. FRAP experiments and image processing were performed same as explained above (see results section 4.2.2.3). By comparing the net protein synthesis in the growth cone like structures from control experiments with cells treated using inhibitors, there is a clear evidence that the protein synthesis is enhanced in the growing tip which is locally regulated and higly polarised depends on the activity status of the growing structures (Figure 14 pic 2,3, Figure 15 pic 1 and Figure 16 pic 3 ) see pointed arrows indiacte the growth cone protrusion with increased ratio. The net increase in protein content upto 5 fold under untreated control condition (Figure 14 A,B,C) is partly due to net anterograde transport. It is also evident from the analysis on nocodazole treated sample (Figure $15 \mathrm{~A}$ ) showing only 2 to 3 fold increase, indicates the inhibition of protein contribution through transport. On the contrary the FRAP analysis of taxol treated (known to cause inhibition of anterograde transport) sample cells, showing 3 to 4 fold increase in net synthesis of protein explains beyond doubt, the presence of local protein synthetic machinery. 


\subsubsection{Analysis on protein folding in cells and in the growth cone.}

\subsubsection{Folding Mutant (FM) can induce Heat Shock Protein 70 in Bag1 transfected CSM cells}

We tested the sensing and induction capability of FM on different cell lines to show the possibility of using FM as a heat shock sensor using a standard biochemical methods. For this experiments the FM YFP driven by CMV promotor was transfected in different cell lines like CHO, CSMwt, CSM BAG1 stable cell line, and CSM BAG1 $\Delta \mathrm{C}$ mutant stable cell line. The cells were grown at $37^{\circ} \mathrm{C}$ for 18 hours and lysed for crude protein lysate. Equal amount of lysate was loaded in a SDS PAGE gel and Western blotting was probed for the total protein using GFP antibody and induction level of HSP70 using Monoclonal HSP70 antibody (Figure21, Blot B). When comparing the level of FM expression to the HSP70 induction, there is a marked increase in HSP induction only in the cells expressing BAG1 but not the BAG $\Delta \mathrm{C}$. The stress response crated by an aggregating FM was mild in the control cells, which was observed slight increase in HSP 70 induction and was observed to be different for the cells like $\mathrm{CHO}$ and CSMwt. (Figure 21, Graph A). The basal level is marked with the control cell line $\mathrm{CHO}$ (Green line Graph1), which show the marked characteristics of CSM cells with and without BAG1 and BAG $\Delta \mathrm{C}$ mutants. The results indicate that BAG1 a co chaperone stably expressed in CSM cells show a dramatic increase in HSP70 induction of 30 fold, which presumably restores the folding capability of FM mutant. The cells expressing BAG del mutant show a negative regulation in HSP70 induction, which was explained in the literatures that BAG del mutant without cterminal HSP binding site acts as a dominant negative regulator of HSP induction (Kermer et al). There is a basal increase in HSP70 level of CSMwt cells as compared 
Figure 21: Response of Folding Mutant induced HSP70 expression in BAG1 expressing cells

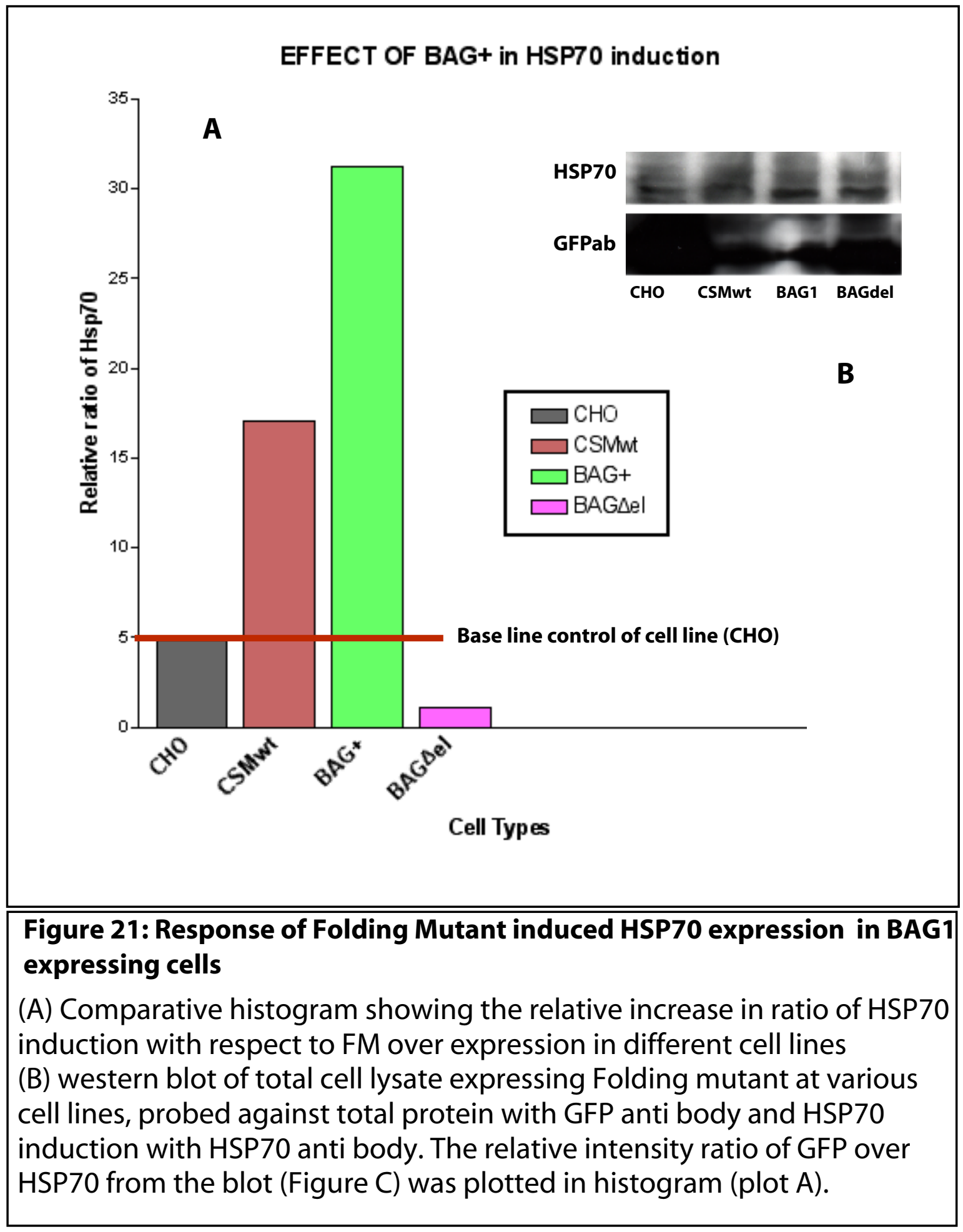


to the $\mathrm{CHO}$ cells, explains the variability in the internal gene expression system and by comparing the level of HSP70 induction with the control of CSMwt cells, BAG1 expressing CSM cells show 10 fold increase in HSP70 induction (Figure 21, Graph A). These results further provide a link that BAG1 is a co-chaperone that induces the level of HSP70 and prevents the degradation of HSP70 immediately after synthesis.

\subsubsection{HSP70 overexpression has no effect on local protein folding at the PC12 cells growth cone}

Our attempt to study the local folding machinery in the growth cone like structures failed to show any indication that there is a heat shock mediated folding in differentiated PC12 cells. The PC12 cells were transfected with HA tagged folding mutant driven by the CMV promotor. CFP-tagged HSP70 was co-transfected folding mutant as a positive control to check for foldase activity. The cells after transfection were grown at $37^{\circ} \mathrm{C}$ for 18 hours and fixed. The HA tagged FM was immuno labelled with CY5 tagged 2' antibody for monoclonal primary HA antibody. To measure the folding efficiency images were taken for YFP emission upon YFP excitation and CY5 emission upon CY5 excitation. By image division of YFP emission with CY5 emission and processing the ratio images of YFP/CY5 for localised folding response. The observation we made, indicated that there is no net difference in folding with and without HSP70. We were unable to find any significant difference in the folding efficiency either in growth cone or in whole cell (Figure 22 graph 1,2 and pic 1,2). The possible argument may be that PC12 cells after differentiation might have a altered response for the induction of heat shock protein and to our surprise there was a supporting evidences came from the work of (Dwyer et al 1996), claims that differentiated PC12 cells fail to respond for the induction of HSP 70 after treatment 
Figure 22 : Local response of HSP70 at the growth cone of PC12 cells

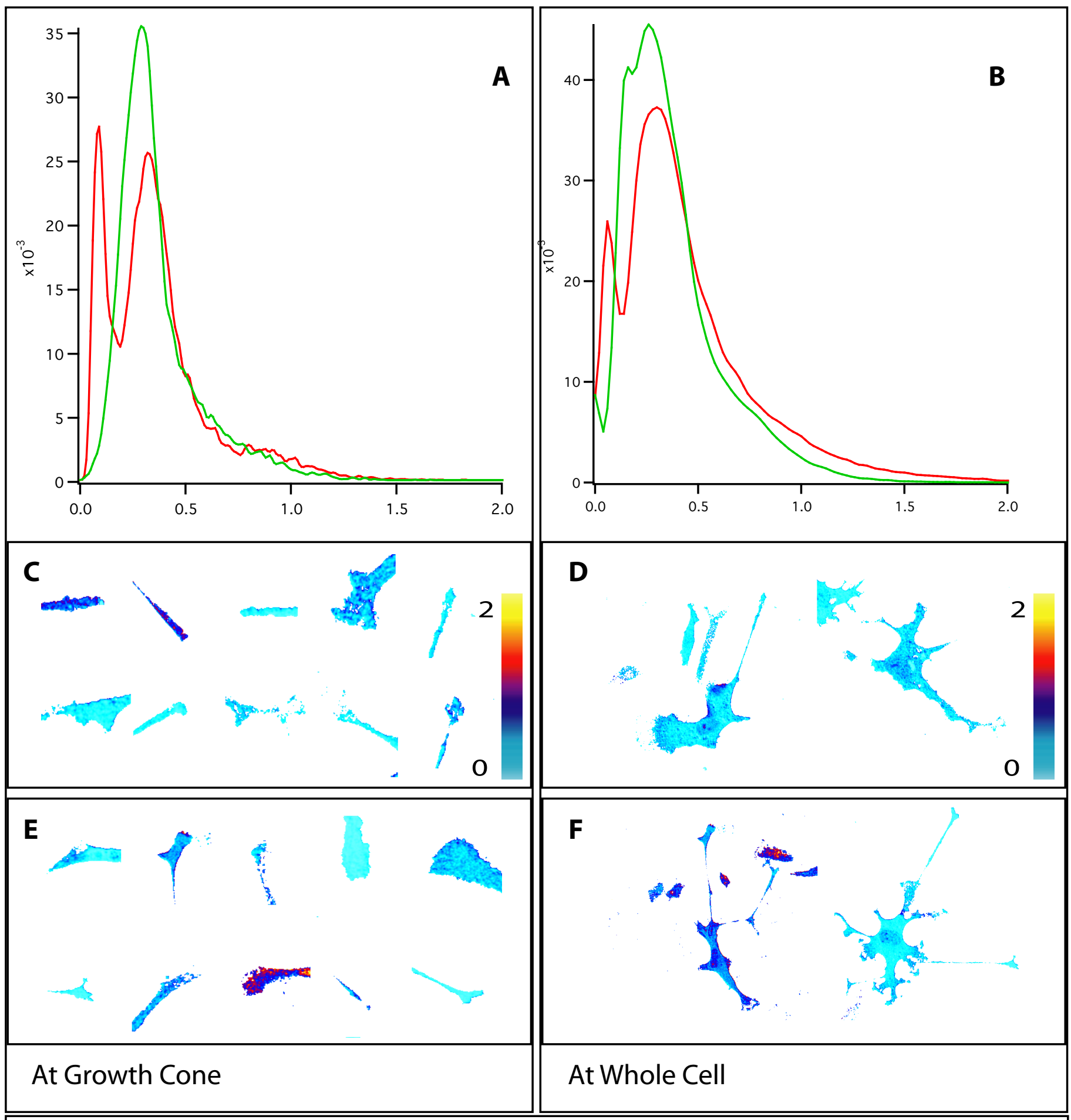

Figure 22: Local response of HSP70 at the growth cone of PC12 cells

$(A, B) F r e q u e n c y$ distribution graph of HSPresponse on folding mutant at growth cone and at whole cell respectively, showing fold increase of positive(Red trace)incomparison to control Green trace). (C,E) Representative pictures plotted in graph for control and positive respectively showing nosignificant increase in positive cells (E) in comparison to control (C). Figures $(D, F)$ Representative pictures of whole cell for HSP induced folding response of folding mutant, showing no significant increase in folding response for positive $(F)$ in comparison with control(F) 
with NGF, while undifferentiated cells induce heat shock response upon brief exposure to elevated temperatures or by addition of ethanol to the cultures.

\subsubsection{Functional analysis of HSP70 in aggregation induced neuropathology}

\subsubsection{HSP 70 overexpression prevents aggregation induced cell death}

To explain over expression of HSP70 in preventing aggregation induced cell death. We used aggregation prone proteins Tau and $\alpha$ Synuclein with and without HSP70 under CMV promotor in CHO cells. To this extent we tested the effect of HSP70 in preventing protein aggregation and cellular toxicity. The GFP tagged cDNA were cotransfected with HSP70 and cultured for 24 hours at $37^{\circ} \mathrm{c}$ and fixed with PFA. We used an In house developed automated Screening methodology (Esposito A et al), to calculate the number of cells for several parameters namely, cell survival, morphology, and increase in intensity. This method allowed us to measure over 6000 cells in each samples and the results are extrapolated with control cells, which is transfected only with normal GFP. The results obtained on the cell count were plotted against the control sample as a ratio of rescue in cell death (Figure 23, Graph A), and it showed a significant rescue ratio for both wt Tau and wt $\alpha$ Synuclein, about 4.5 and 2.5 fold respectively, By looking at the rescue ratio of Mutant situation is rather less significant with respect to $\alpha$ Synuclein mutants and mild or less dramatic with Tau mutant when compared to wild-type situation. These differences in rescue ratio can further be counted as a indication of severity in toxicity of mutant with respect non aggregating wild type and their response to stress signal activation. 
Figure 23: Effect of protein aggregation in HSP70 induction and cell survival

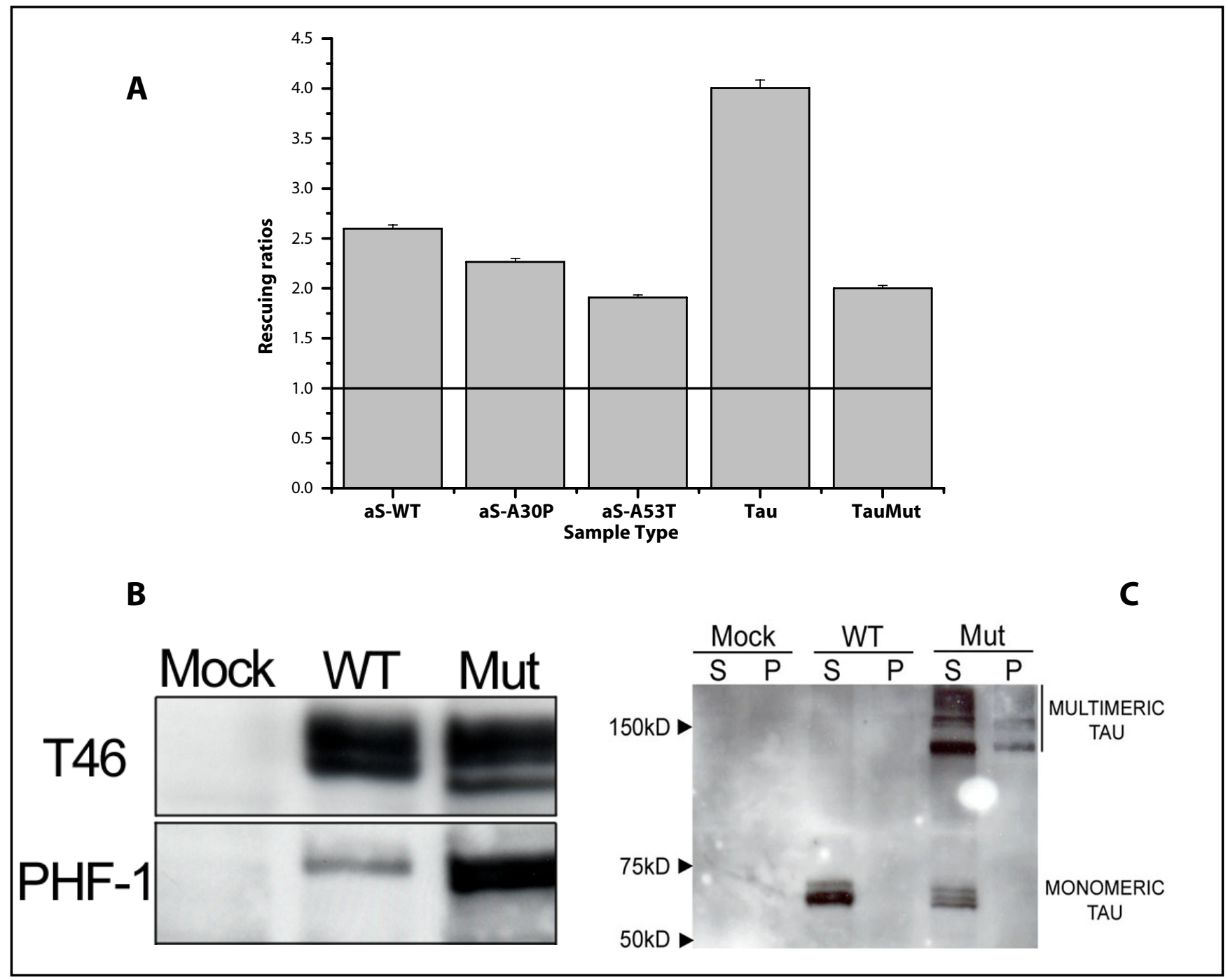

Figure 23: Effect of protein aggregation in the HSP70 induction and cell survival (A) Bar graph showing effect of protein aggregation in cellular toxicity and the resultant rescue ratio of HSP70 over expression on cells expressing aggregting proteins. control line indicates the base line with GFP and HSP70 co-expression condition (Figure A black line) (B) western analysis on strength of protein phosphorylation with aggregating Tau mut protein in comparison with Tau wt,proteins are probed with T46 (C-terminal antibody) and PHF-1 (phosphorylation antibody) to show the amount of protein and relative phosphorylation (C) Western analysis of Tau mut protein aggregation in comparison with Tau wt, probed using C-terminal anti tau antibody showing multimeric tau aggregates in supernatant and pellet fraction of Tau mut but not in the wt Tau or mock situation 


\subsubsection{Protein aggregation and cyto-toxicity are induced by phosporylation}

We further tested the strength, physiology and the severity of fast aggregation in Tau pathology. We performed western blot based biochemical analysis on the Tau wt and Mutant protein expressed under physiological cell system. To perform the experiment we choose the $\mathrm{CHO}$ cells, which lacks the endogenous source of Tau and hence the aggregation and toxicity is mainly based on the extracellular source of Tau expressed under a controlled mammalian CMV promotor. Western blot analysis was performed on protein samples, expressed and harvested from $\mathrm{CHO}$ cells. Fractions were separated into soluble supernatant and insoluble pellet fraction; this is to show the level of aggregation and their soluble properties upon aggregation. Samples were probed with primary antibody against C-terminal 41 aa sequence of Tau. From the results to our surprise the Tau mutant was found primarily on the soluble fraction with a tetramer adduct of Tau protein with small fraction of it in the monomer form. It was also quite clear from the blot that there is a significant proportion of multimer Tau protein in pellet fraction (Figure 23, Blot B), which indicate that the aggregation is due to increased specific protein to protein interaction induced by phosphorylation than a non specific hydrophobic interaction and it might have a link with hyper phosphorylation of Tau protein with aggregation. This idea was supported by the evidence came upon the western blot analysis of Tau protein for phosphorylation, by using classical Tau antibodies, which labels the phosphorylated amino acids. Antibody against Tau paired helical filament (PHF) (Figure 23 Blot C) showed a high molecular weight adducts with strong antibody labelling when compared to wild type Tau protein, The strength of phosphorylation would be atleast 10 fold excess in Tau mutant when compared to Tau wt. 


\section{Chapter 5: DISCUSSION}

\subsection{The use of biosensor for the study of local events of protein transport, ubiquitination, folding and synthesis.}

\subsubsection{Development of axonal transport marker to study the contribution of cell soma for local protein pool in the growth cones}

From our current investigation, it is clear that the transport of synaptophysin (p38) FLAP tag in the axon is bidirectional and the total protein as a bulk is transported both in anterograde and retrograde direction. By looking at the amount of protein transported, we record that about $60 \%$ of the protein from the bleached region is primarily transported in the anterograde direction and $30 \%$ of it in retrograde direction. This enable us to measure the amount of protein transported locally toward the distal region of axons for instance the growth cones.

Above all, by looking at the relative location of synthesized protein and the protein transport using this assay, we record the directionality, bulk of protein in specific direction, and the relative time frame in which protein transported. This assay suggests that FLAP tag based transport marker can be used as a relative index for the proteins transported, the direction in which they are transported and the amount of protein transported in specific time. By tagging the candidate protein to FRET based FLAP sensor, we have an ideal tool to study the subjective contribution of cell soma to the protein pool of the growth cone and these informations discussed above may 
eventually contribute for the information of local protein turnover mecanism in growth cone navigation.

\subsubsection{REACh-based FRET sensors for protein ubiquitination}

We demonstrate by different methods the suitability of the REACh acceptor in determining FRET between proteins in a physiologically relevant setting. The novel FRET pair GFP-REACh was used to construct a very sensitive assay for the visualization of protein ubiquitination in single cells, both by FLIM-based method and by a modified FqRET method on a dual-dye ubiquitination substrate. In its current use, the ubiquitination assay reports on the local activity of the ubiquitination machinery, but can easily be expanded to detect the ubiquitination of other GFPfusion proteins and can be converted into a cell-based screening assay for candidate protein substrates that possibly be ubiquitinated

\subsubsection{REACh-Based Ubiquitination assay in biological systems}

Our study using REACh based ubiquitination assay has innumerable advantages like, detecting ubiquitination of proteins involved in physiological signalling cascade, measuring the recycling rate of protein targeted to ubiquitination and the physiological interaction (protein assisted-protein degradation) of the proteins targetted to the proteasome mediated degradation. In the current assay we measured the protein ubiquitination by using de-stabilised GFP with REACh labelled ubiquitin. By simply replacing the GFP-PEST with other known or possible protein ubiquitination substrate with GFP donor, it is possible to measure the active protein ubiquitination of any candidate protein. With this we also provide two independent 
possibility of FRET measurement namely, FRET as measured by FLIM in time correlated single photon counting (TD-FLIM) and intensity dependent measurement of donor quenching using confocal based FqRET. Furthermore this protein ubiquitination probe can be used in cultured neurons to detect and locate the functionally active protein ubiquitination machinery in vitro.

\subsubsection{Spectral advantages of REACh}

Most of the spectral and practical advantages are due to the loss of detectable fluorescence from the acceptor. It should be noted, however, that with the current mutants this holds true only for two-photon excitation. When imaged under single photon excitation conditions, very weak residual fluorescence from these REAChes can be observed. This has no practical consequences for the ratiometric FqRET method, but does contaminate the lifetime measurements on our other, wide-field frequency-domain, FLIM setup. Apparently, the absorption characteristics of REACh under one- and two-photon conditions differ dramatically and the two-photon crosssection appears to be very low in comparison. In this respect, contribution of lower lifetime emission from the residual fluorescence of REACh can only be caused by direct excitation as sensitized emisssion follows the decay characteristics of the longer lifetime GFP donor.

Since most commercially available and practically implemented FLIM equipment in use today is based on the femto-second-pulsed nature of an available two-photon laser, allowing an existing scanning two-photon microscopy setup to be easily adapted for FLIM measurements by relatively inexpensive photon counting electronics and an additional sensitive photon point detector, the REACh mutants described here will be 
Figure 1: Advantages of REACh mutants in FRET based measurements

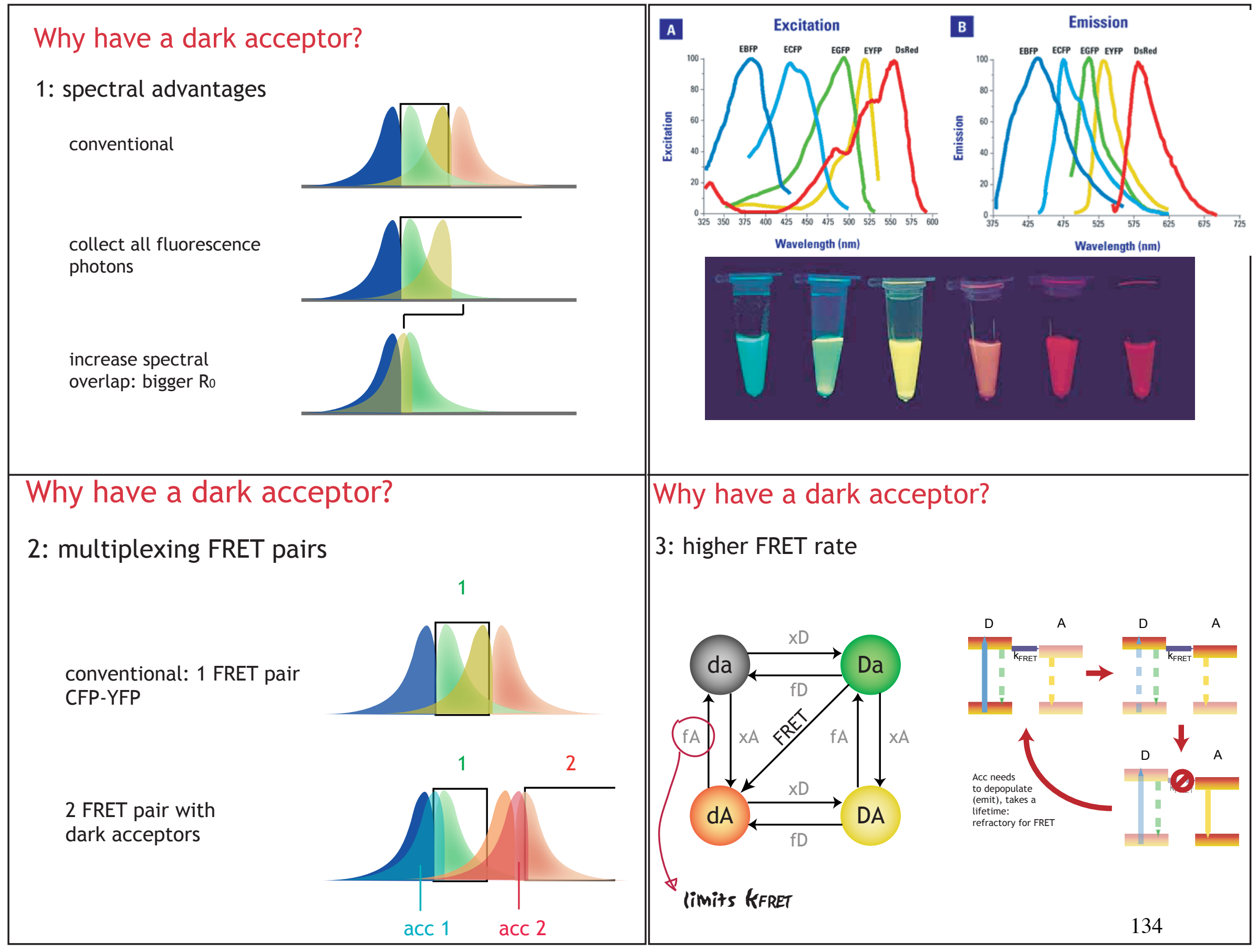


of direct use for most FLIM users. Although the REACh acceptor is not visible in cells, the meaningful readout of FRET measurements is the visualization of the donoracceptor complex. This is inferred from the occurrence of FRET as judged from the donor fluorescence properties. In fact, in many FRET assays, the acceptor is commonly present in large excess over the donor in order to saturate the donor binding event for optimal and quantitative detection of the interaction.

For most questions, as for our ubiquitination assay, the localization of non-interacting acceptor-labeled proteins does not carry much information. When required, the localisation of the acceptor can be established either in a separate experiment with a fluorescent acceptor or in a post-acquisition determination e.g. using antibodies against an epitope-tagged acceptor construct similar to our additional fluorescent modification of the donor-labeled molecules for the FqRET implementation. Alternatively, the acceptor could be doubly tagged with the spectrophysically superior REACh acceptor and a (far-) red emitting fluorescent protein for the simultaneous establishment of its localization in a FLIM experiment. Our experiments demonstrate the applicability and the advantages of the use of dark chromoproteins as FRET acceptor for GFP. This FRET pair GFP-REACh is superior to the currently favoured CFP-YFP combination due to its larger overlap between donor emission and acceptor absorption spectra and enables the use of fluorophores that are not separable otherwise. REACh also has a number of advantages over previously reported FRET methods and pairs. 


\section{REACh: Resonance Energy Accepting Chromoprotein}

construction of a live-cell ubiquitination assay

Bright Half Bright REACh
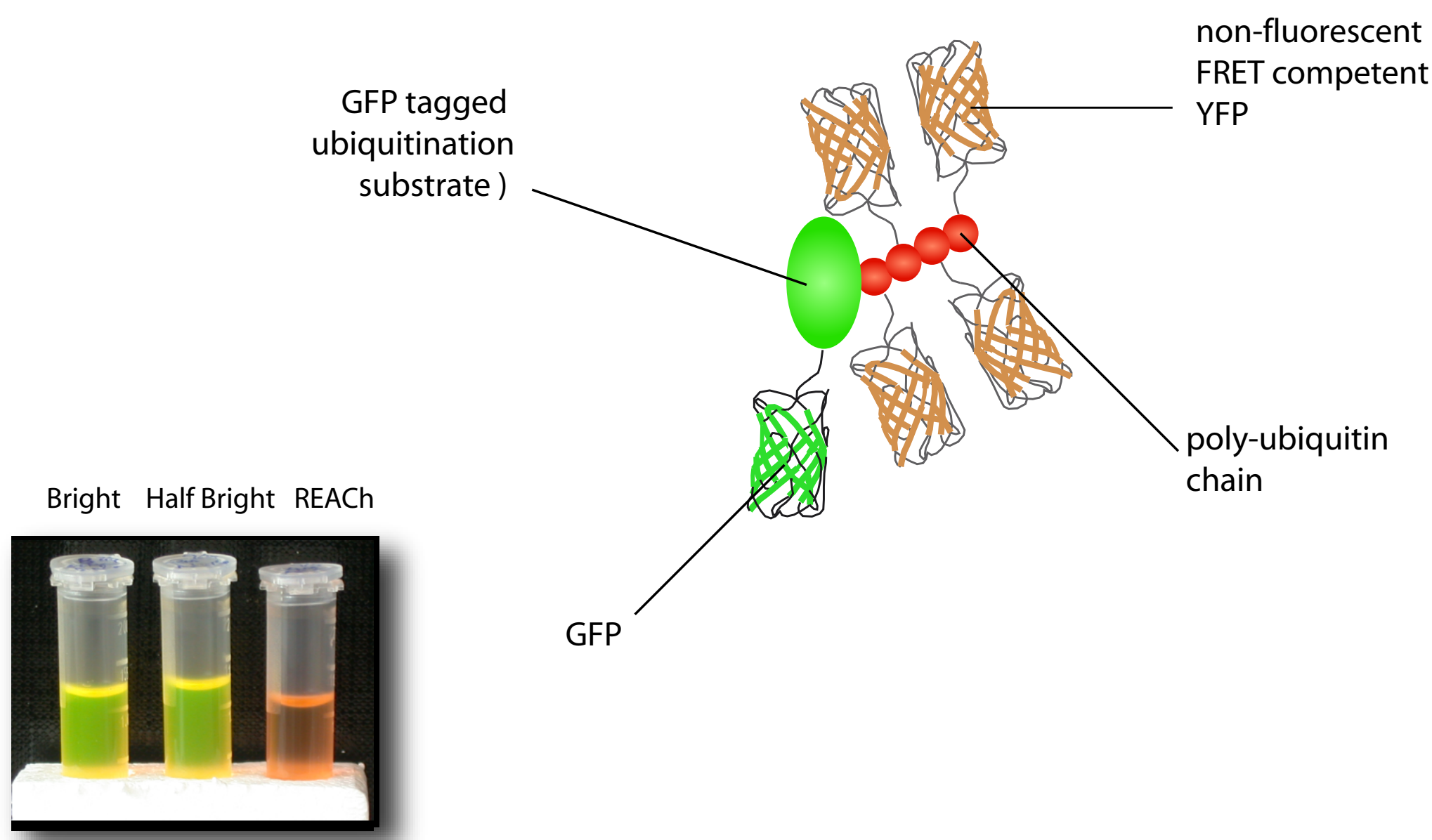


\subsubsection{Advantages of REACh acceptors for FRET based measurements}

Since our dark REACh mutants retain their absorptive properties, they are ideal acceptors for FRET. The advantage of REACh acceptors in donor-based FRET measurements like FLIM and donor photobleaching FRET, is that every emitted photon from the pair contributes to the FRET measurement, thus obviating the need for strict spectral filtering and thereby increasing the signal-to-noise ratio. In our FqRET assay, the Cy5 reference fluorophore is positioned in a part of the optical spectrum that effectively also allows the collection of the entire emission band of GFP. Furthermore, REACh acceptors allow a larger spectral overlap of donor emission and acceptor absorption, thus increasing the separation distance over which FRET can be detected. This is obvious from the calculated R0 distances of the REACh-GFP pair (5.6 and $5.9 \mathrm{~nm}$ ) which exceed the R0 distance of the currently favored CFP-YFP couple (4.9 nm) (Harpur, A.G., et al 2001) .

The extremely short excited state lifetime of the REACh acceptor provides an additional advantage to FRET measurements. Since an acceptor molecule in the excited state cannot accept energy from a donor, the lifetime of the acceptor limits the rate of FRET16. Dark, short-lifetime fluorophores, i.e. chromoproteins, are therefore photophysically preferred as FRET acceptors over their fully fluorescent counterparts. Finally, another advantage of the extremely short fluorescence lifetime of the REACh chromoproteins is that acceptor photobleaching via FRET-mediated excitation is abolished. High FRET rates and an unfavourable photostability balance between the donor and acceptor can lead to serious degradation of the measured FRET efficiency. Because the use of a dark acceptor liberates a large part of the optical spectrum, an additional advantage of REACh is that it enables the use of fluorescence-based 


\section{Intensity-based FRET using a dark acceptor}

by application of a concentration reference dye
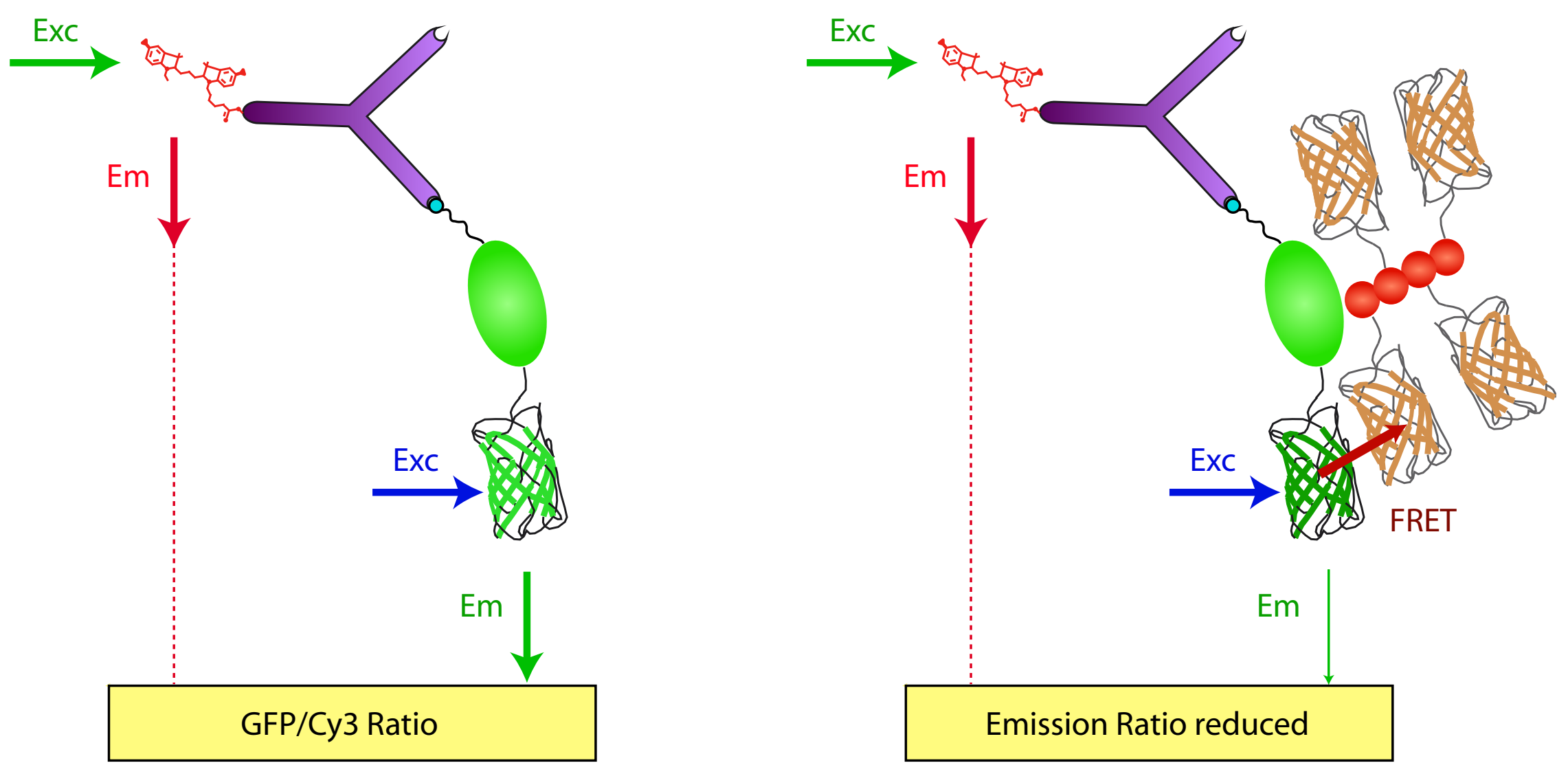
detection of additional cellular components or even multiple FRET pairs simultaneously (FRET multiplexing). In the latter case, the multiple donors need to be suitably spectrally separated and used with suitably corresponding dark REACh acceptors. An increasing number of non-bioluminescent coral-derived chromoproteins have been described that were rendered fluorescent by mutagenesis. However, the far red-emitting variants could be used in their original non-fluorescent forms as acceptors for red-emitting VFPs to form a second FRET pair alongside GFP-REACh in the same cell. Other spectrally isolated combinations will likely become available from alternative sources for novel spectral variant fluorescent proteins with improved spectral properties (Fradkov, A.F. et al., Yanushevich, Y.G. et al 2002).

The possibility to correlate a FRET effect with the behavior of another fluorescently labeled cellular component allows the generation of sophisticated bioassays that will be needed to probe causal connections in the biochemical network that governs cell behavior. Furthermore, in the case of the currently popular donor-acceptor chimeric FRET sensors, our approach can transform ratiometric methods into a simple singlewavelength intensity measurement. In addition, the use of only one spectral band in the determination is of advantage to simplified instrumentation for FRET detection, e.g. in high-throughput screening. The unique possibilities that the REACh method offers will expand the applicability of current FRET biosensors by improving the sensitivity, design and flexibility of both FLIM-based and intensity-based FRET assays. 


\subsubsection{Biosensor to detect the chaperone assisted local protein folding}

\subsubsection{Protein folding-dependent foldase sensor}

In the current study to detect the local event of structural instabilty of proteins as the mechanism of regulation of growth cone navigation, we created a novel biosensor that can sense the folding discrepancy and stress induced heat shock protein induction. This sensor work with the principle of folding dependent increase in the fluorescent intensity and we call it as Foldase sensor. To study the functional role of foldase sensor we used a system that induce the heat shock protein expression that inturn results in folding of foldase sensor and it has the direct relevance to neuronal injury and cell survival.

\subsubsection{Foldase sensor in sensing BAG1 induced HSP70 induction}

BAG1 has been characterized as a regulator and marker of neuronal differentiation exhibiting neuroprotective properties in vitro as well as in a transgenic mouse model in vivo (Kermer et al. 2002 and 2003). This multifunctionality has been suggested to be dependent on the binding partners interacting with BAG1 (Takayama and Reed 2001). To explore the influence of the known binding partner HSP70 on the above mentioned features of BAG1, in the present study, we stably overexpressed a BAG1 deletion mutant (BAGdelC), lacking Hsp70 binding capacity. We used CSM14.1 cells, which originate from rat nigro-striatal neurons immortalized by expression of temperature-sensitive SV40 large T antigen. These cells show a high proliferation rate at permissive temperature, while inactivation of large $\mathrm{T}$ antigen at non-permissive temperature induces neuronal differentiation (Zhong et al. 1993). 


\subsubsection{Foldase sensor in sensing the differences of inducibility of chaperone}

It is known that chaperones interact with misfolded or partially folded polypeptides in order to prevent interactions that would result in aggregation. Chaperones belonging to the Hsp- and Hsc70 (70-kDa heat shock protein and cognate protein) family handle the majority of substrates within the eukaryotic cytosol. The main function of these chaperones is to fold newly synthesized proteins or to refold proteins after stress denaturation through ATP binding and hydrolysis (Bukau and Horwich 1998; Hartl and Hayer-Hartl 2002; Young et al. 2001). The subcellular localisation as well as the substrates of such chaperones are predominantly determined by a number of regulatory or accessory co-chaperones. In general, such co-chaperones consist of a chaperone-binding domain and other domains which supply different activities or localisations within the cell. BAG1 is one of those known co-chaperones. It has a Cterminal BAG-domain which interacts mainly electrostaticly with Hsp70 (Sondermann et al. 2001). BAG1 binds to the ATPase domain of Hsp70 resulting in a strong acceleration of ATPase activity (Höhfeld and Jentsch 1997). Further investigations revealed that this acceleration is due to stimulation of ADP release via opening of the nucleotide binding cleft of the Hsp70 ATPase domain (Höhfeld and Jentsch 1997; Gässler et al. 2001; Sondermann et al. 2001). BAG1 thereby acts as nucleotide exchange factor for Hsp70, triggering substrate unloading from the chaperone. Though the mechanisms of BAG1/Hsp70 interaction were studied extensively, the influence of BAG1 on chaperone 'foldase' activity is still a subject of controversy and debate. While some studies suggested BAG1 to be an inhibitor of the ATP-dependent Hsp70 activity (Gebauer et al. 1997; Takayama et al. 1997; Zeiner et al. 1997), others presented strong opposing evidence for BAG1 as a positive co- 
chaperone (Terada and Mori 2000; Gässler et al. 2001). These opposing findings were attributed to differences in co-factor expression, but also by the expression of different BAG1 isoforms with the short forms of BAG1 acting as positive, and longer forms as negative regulators of chaperone foldase activity (Lüders et al. 2000).

In this study, we demonstrate that the short isoform of mouse BAG1 dramatically increases chaperone foldase activity. To our knowledge, we are the first to demonstrate and quantify BAG1-dependent chaperone activity inside single cells.

\subsection{Observations on protein turnover in the growth cone}

Protein turnover or recycling as a regulatory mechanism in the axonal growth cone navigation is still considered to be a premature field of research. Though there are many research publications, supporting the theory of axonal protein synthesis and degradation, there is no systemic evidence yet, to support the role local protein turnover mechanism in regulating the growth cone navigation. In this study we investigated the combined roles of local protein turnover mechanisms namely transport, synthesis, degradation and folding in regulating the axonal growth cone morphologic behaviour in the context of axonal pathfinding.

\subsubsection{Protein translation and degradation occurs locally in the growth cone}

In this work, we investigated the combined roles of local translation and degradation during growth and collapse of neuronal growth cones. We observed that when 
microtubule mediated transport is inhibited in cells using taxol or nocodazole, the bleached regions of growth cone like structures of pc12 cells expressing NFM-GFP indicated a marked increase in total protein content of GFP-tagged NFM protein content within $30 \mathrm{~min}$. The fold increase in protein content can be taken into consideration as a rough index for growth cone protein requirement under different stimuli and the net synthesis of protein in use upon specific stimulus. In this work we identified the independent activity of growth cone protein synthesis by blocking the transport of protein from cell body towards growth cone or away from it. The rate in which the protein synthesis occurs, provides the insight that the growth cone has its own protein translation machinery and it continuously replenishes the local pool of protein required during activity. It is also evident that growing axonal growth cone tends to show a dramatic increase in protein synthesis at the leading edge in comparison to the growth cone central domain suggesting that accumulation of newly synthesized protein at the leading edge is needed for growth promotion. On the other hand we also found that growth cones of pc12 cells with collapsed morphology show reduction in their local protein level in short duration of titme. We found a significant reduction in the total content of protein within 30 min after blocking the microtubulebased transport, suggests that protein degradation is a local event and it indicates that growth cone follows one of these recycling mechanism to make autonomous decisions upon growth and collapse under different stimuli.

In the other findings with REACh ubiquitination assay performed in differentiated PC12, we found it noteworthy that protein ubiquitination machinery can be a part of local pool of growth cone protein recycling machinery. From the REACh-based FqRET and FLIM measurement in PC12 cells for protein ubiquitination, most of the 
micro clusters positive for ubiquitin antibody are localised around axonal periphery, along axons and in branches, indicative of the presence of ubiquitination machinery in growth cone and hence activated and regulated in close proximity to the growth cone. With all the convinsing evidences of local translation and degradation, it is still hard to exclude the concept that axonal transport may play a major role in contributing for the significant proportion of protein recycling. The anterograde transport of mRNA and organelles is beleived to be responsible for sequestered synthesis of protein of interest at any given situation during growth cone navigation (Roy et al., 2000; Wang et al., 2000; Wang and Brown, 2001). On the other hand there is still a debate whether retrograde axonal transport actually helps maintaining the local pool of recyclable protein content at the growth cone. This leads us to the conclusion that transport cannot be excluded completely from the local mechanism of regulation for chemotropic growth cone guidance.

\subsubsection{Chemotropic response of neuronal growth cone mediated by rapid local protein turnover}

We have examined the role of protease and proteasome-dependent proteolysis in mediating chemotropic responses of cortical neuronal growth cones. We found that each of the three different cues investigated, namely sema3a, LPA and netrin1 elicits responses through intracellular pathways that involve either translation or degradation or a combination of both. Sema3A and LPA-induced responses require degradation, while netrin-1-induced growth or repulsion is altered by protein degradation, but not so significantly. Together, our data suggest that growth cone steering is likely to be mediated by rapid local changes in protein levels. 
In our experiments, pharmacological inhibition of the proteasome blocks both netrindependent growth cone turning and LPA and sema3a -induced growth cone collapse. In our experimental conditions, inhibitors seem to increase the branching of cortical neurons which are induced by Netrin1 and tend to stabilise the normal growth cone morphology in the case of sema3a or LPA, that are known to induce collapse. Since the local protein translation is also required for appropriate growth and dynamics of the growth cone, the guidance cues may presumably induce chemotropic guidance through the regulation of protein levels. Within the growth cone there must be a protein turnover machinery to regulate the balance of protein synthesis and degradation for a rapid changes in local protein composition. With all the above evidences mentioned above, involving local protein turnover in the growth cone, it provides us with the clue that local protein turnover can be considered a powerful regulatory mechanism controling the growth cone behaviour locally. Furthermore our findings reveal similarities between axonal guidance and synaptic plasticity through the role of ubiquitin-proteasome system. Ubiquitin-proteasome system has been implicated in axon guidance (Aguilera et al., 2000; Muralidhar and thomas, 1993; Oh et al., 1994; Poek et al 2001), and synaptogenesis (Di Antonio., et al 2001). Our findings suggests the presence of ubiquitin-proteasome system in the closed growth cone compartments of PC12 cells and the possible role of it in the growth cone guidance on chemotropic responses of the growth cone.

A similar study by Campbell \& Holt using Xenopus retinal growth cones demonstrates that ubiquitin-dependent proteasomal degradation is part of the answer (Campbell \& Holt 2001) for chemotropic response of growth cone. The growth cones 
of Xenopus retinal neurons in culture contain ubiquitin, the E1 ubiquitin-activating enzyme, and proteasomes. Netrin-1, semaphorin, 1-a-lyso-phosphatidic acid (LPA) is known to be the guidance molecules that use the ubiquitination machinery to regulate the behavior of these growth cones. The work of Koenig 1967; Bassell et al. 1998; Eng et al. 1999; Koenig et al. 2000 and Campbell and Holt 2001, demostrate that within five minutes of encountering netrin1 or LPA, the levels of ubiquitin-protein conjugates in the growth cone doubles.

\subsubsection{Chaperone induction is required for neuronal survival}

From the extensive use of foldase sensor in detecting HSP70 induction, we infered an interesting finding that chaperone induction is responsible for cell survival and morphological changes of cells under stress. Protein aggregation reponsible for stress activation and cell death induces HSP70, thereby prevents the cell death. The chaperone protects cells by reducing the amount of cellular aggregate or increase the accecibility of misfolded protein for proteasome mediated degradation. In other words aggregation of protein prevents proteasome mediated degradation which inturn increases cellular stress mediated HSP70 induction (Bence FN., et al 2001). In our experiments with folding mutant, we designed an assay to check the local chaperone activity; where in, the chaperone induction is sensed by the direct measure of intensity changes either from Folding mutant or CY3.1 tagged antibody against HSP70. This measure is a responsive index for the involvment of chaperone mediated stress activation. In our assay system, we checked the roles of heat shock protein in preventing aggregation induced cell stress, by the cytoskeletal and microtubuleassociated aggregation-prone proteins alpha-synuclein and tau respectively, we observed the role of chaperone in preventing aggregation-induced local stress 
response. By dissecting the internode of chaperone activation and stabilisation using BAG1 sensitive folding assay, we further provide a strong link for chaperone-induced prevention of cellular toxicity via anti-apoptotic cascade. Since the chemotropic response induced by growth cone guidance cue is one such phenomenon, which could possibly influence the local folding machinery through stress related cascade, the chaperone sensitive folding assay can further be used to check these local responses at growth cone microenvironment. Furthermore by using the chaperone sensitive assay for local changes in chaperone induction and folding, we created an essential tool to study the stress-related activity induced by chamotropic guidance cues in neuronal cell systems. To this effect we are yet to study the response of other heat shock protein sub-families, that are presumably involved in heat shock response namely HSP27 and HSP90. These observations on local protein transport, synthesis, degradation, and folding, which are critically important to the growth cone responses to chemotropic cues, we put forward the follwing interesting questions to be studied in the future;

1.Which protein(s) other than NFM and spectrin are transported, synthesized, folded and degraded? Candidates might be proteins such as the cytoskeletal-anchor proteins or cytoskeletal-associated proteins

2. Which is the regulatory intermediate key component, maintaining the non-toxic level of protein under different stimuli of guidance cues? (a) Ubiquitin-proteasome machinery (b) Intrinsic protease-mediated degradation or (c) Chaperone-mediated folding/unfolding

3. Which is the intermediate step between chemotropic response of the growth cone? (a) Synthesis vs Folding (b) Misfolding vs Degradation or (c) Transport vs Synthesis. 


\subsection{Conclusions and implications of local protein turnover}

\section{as a regulatory mechanism for growth cone function}

1. As argued above, proteins are transported along the axons in a rather slow rate, axonal transport can therefore not be sufficient to supply necessary proteins to the growth cone in a time frame required to explain the morphological changes in the growth cone. On the other hand, retrograde axonal transport could contribute to the rapid clearance of the proteins as the growth cones represent a small confined volume. Of course, we cannot exclude the anterograde delivery of proteins to the growth cones from a distal axonal pool. Instead,

- We provided evidences for protein synthesis within the local growth cone environment, independent of the transport. Since the local growth cone protein synthesis rate is clearly detectable within very short time frames (5-10 min), after blocking the transport, it might be the immediate adaptation response of the growth cone to the effect of guidance cues. In contrast, the much slower axonal transport-mediated response aids the long term fate of the growth cone.

2. The presence of proteasome components in the growth cones suggests that proteins can be recycled and cleared, depending on need. As both of these mechanisms; ribosomal protein translation from axonally delivered mRNAs and the ubiquitin proteasome system, present tonically active machineries, increased clearance, production, or recycling rates of specific proteins demands the necessity for regulation of the protein cycle at the level of chaperones. Chaperones are positioned at the ideal 
location to control protein levels as their inactivity would cause newly produced nascent polypeptide chains to be shuttled to the proteasome directly, while their activity would lead to the folding and therefore functional stabilization of specific proteins. In order for this cycle to work properly in response to environmental cues and to the metabolic status of growth cones, chaperones need to be regulatable other than through their synthesis. This regulation necessarily has to be embedded in the cellular signaling network in order to respond to external stimuli. To this speculation,

- We observed the local interaction (PESTgfp+REAChubq) in the growth conelike structures of PC12 cells. Furthermore our collapse assays experiment showed a reversible response of collapse to growth for proteasome-mediated chemotropic response in cortical neurons. This provides us a conclusive evidence for proteasome and protease-mediated protein turnover machinery in the growth cone.

3. Acute changes in chaperone activity would lead to the desired changes in the composition of the local growth cone proteome. Given the morphological changes of the advancing growth cone, the proteins that are likely under control of these regulatory mechanism include, cytoskeletal associated and structural proteins. The BAG1 protein is a regulatory protein that controls HSP70 activity, it signals downstream through the RAF kinase, ERK pathway, which are involved in differentiation, and is itself under regulatory control of an upstream anti apoptotic signaling network through its connection to the Bcl-2 protein family. Disturbances in this tightly regulated cycle are also likely to be involved in neurodegenerative conditions, were aggregation of proteins like poly-glutamine proteins, microtubule 
binding protein tau, and alpha synuclein, lead to neurotoxicity in huntingtins disease, Alzheimer's disease, and Parkinson's disease respectively.

- We observed in neuronal cultured cells, the role of BAG1-induced chaperonemediated processes like, protection, regeneration and degeneration under stress-related conditions. Since chemotropic response is one of that kind yet to be studied extensively, we link the role of chaperone-assisted folding as a crossroad between protein synthesis and proteasome-dependent degradation. This theoratical explanation could be applied to the role of local induction of stress related proteins in mediating axonal growth guidance and neuronal development. 


\section{Hypothetical model for local protein turnover: Project Overview}

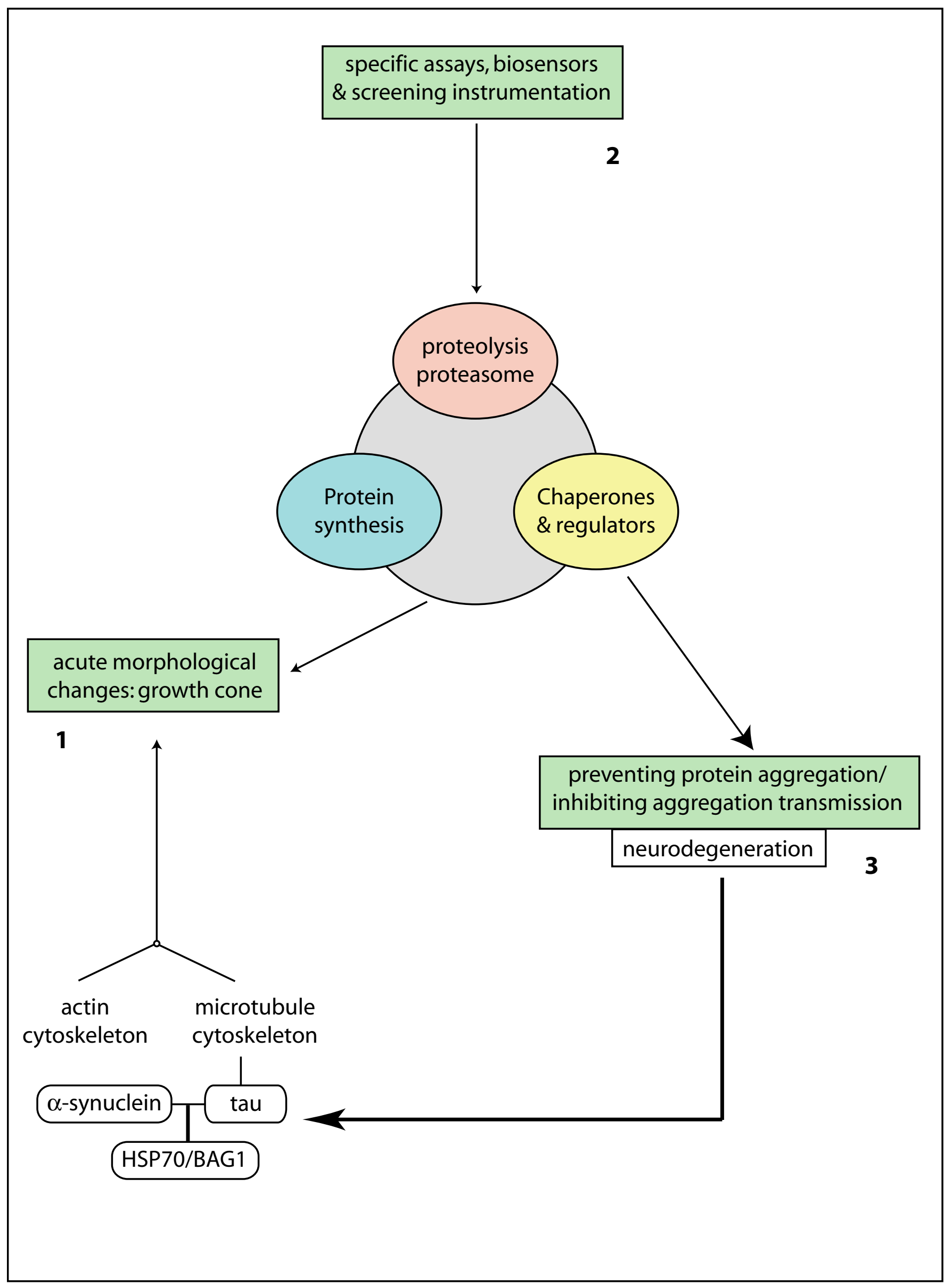




\section{Summary and conclusion}

Axonal growth cone of neurons contains protein synthetic and degradation machinery. The growth cone synthesize proteins locally, which depends on their need and the influence of local chemotactic response induced by guidance cues. The growth cone collapse of cortical neurons induced by LPA and sema 3a require protein degradation machinery and the collapse was completely reversed to growth, by inhibiting both proteasome and protease mediated protein degradation. On the contrary, netrin 1 induced growth or branching remains unaltered and showed no inhibition with proteasome or protease inhibitors. All the evidence obtained from collapse assay, suggests that growth cone guidance is mediated locally by several protein turnover machineries, namely proteasome, proteases and translation machinery. The presence of endogenous protein-synthesizing machinery in axons and nerve terminals creates an entirely new perspective in studies of the biology and pathobiology of neurons. Processes like, growth, differentiation, maintenance, plasticity, pathobiology of axons and nerve endings should be viewed in the context of local mechanisms, which allow axons and nerve terminals with the capacity to respond in a semi-autonomous manner to local challenges.

The growing axonal growth cone contains protein synthetic machinery and the rate in which protein is synthesised is purely based on the activity and need of the growing axonal growth cones. Axonal growth cone contains protein ubiquitination machinery, which provides the link for recycling of proteins after synthesis to maintain the local pool of protein under non-toxic level. The functions of ubiquitin in the nervous system is considered to be central for modifiying neuronal activity and is known that 
ubiquitin can alter the funtion of neurotransmitor receptors and ion channels. It is likely that ubiquitin regulates short term plasticity and neuronal excitability and holds the key to specificity in the nervous system by destryoing abnormal proteins, crucially involved in development and fuction of the nervous system. Our ubiquitination-based assay on FRET technology, provides a novel tool to study these functional role of ubiquitin in the axonal guidance under the influence of guidance cues.

Our foldase sensor, with its ability to sense the heat shock protein induction, presents a promising tool in the field of chaperone mediated stress response. With the combined use of folding mutant and the chaperone assay, it becomes possible to identify the role of protein folding machinery in the context of local protein turnover mechanism. In this current research, by identifying the role of BAG1 as an antiapoptotic factor in regulating the level of heat shock protein and HSP70-mediated cell survival we provide a strong link for chaperone induced protein folding and cell survival. The chaperone assay provides a promising tool to study the role of chaperones in preventing the aggregation, stability and folding of the proteins in the growth cone microenvironment, which is altered by chemotropic guidance molecules. By sensing the ability of guidance cues in promoting stress-related cascade and the regulatory role of heat shock protein in the local protein regulation, it brings us a step ahead to study extensively the role of heat shock protein in protein-folding, preventing aggregation and aggregation- mediated neuro-degenerative diseases. With these concluding remarks we further speculate that we are one step closer to the study of chaperone-mediated local folding response as a key internode in chemotropic guidance of axonal growth cones. 


\section{Chapter 6: BIBLIOGRAPHY}

- Albani, D., E. Peverelli, et al. (2004). "Protective effect of TAT-delivered alpha-synuclein: relevance of the C-terminal domain and involvement of HSP70." Faseb J 18(14): 1713-5.

- Alberti, S., Esser, C. and Hohfeld, J. (2003) BAG-1--a nucleotide exchange factor of Hsc70 with multiple cellular functions. Cell Stress Chaperones 8: $225-231$

- Alder, J., H. Kanki, et al. (1995). "Overexpression of synaptophysin enhances neurotransmitter secretion at Xenopus neuromuscular synapses." J Neurosci 15(1 Pt 2): 511-9.

- Ambron, R. T., J. E. Goldman, et al. (1975). "Effect of inhibiting protein synthesis on axonal transport of membrane glycoproteins in an identified neuron of Aplysia." Brain Res 94(2): 307-23.

- Ancolio, K., C. Alves da Costa, et al. (2000). "Alpha-synuclein and the Parkinson's disease-related mutant Ala53Thr-alpha-synuclein do not undergo proteasomal degradation in HEK293 and neuronal cells." Neurosci Lett 285(2): 79-82.

- Andersen, J. B., C. Sternberg, et al. (1998). "New unstable variants of green fluorescent protein for studies of transient gene expression in bacteria." Appl Environ Microbiol 64(6): 2240-6.

- Andorfer, C., Y. Kress, et al. (2003). "Hyperphosphorylation and aggregation of tau in mice expressing normal human tau isoforms." J Neurochem 86(3): 582-90.

- Andreatta, C., P. Nahreini, et al. (2001). "Use of short-lived green fluorescent protein for the detection of proteasome inhibition." Biotechniques 30(3): 65660 .

- Arai, Y., M. Yamazaki, et al. (2001). “Alpha-synuclein-positive structures in cases with sporadic Alzheimer's disease: morphology and its relationship to tau aggregation." Brain Res 888(2): 287-296.

- Aronov, S., G. Aranda, et al. (2001). "Axonal tau mRNA localization coincides with tau protein in living neuronal cells and depends on axonal targeting signal." J Neurosci 21(17): 6577-87.

- Avila, J. (2000). “Tau aggregation into fibrillar polymers: taupathies.” FEBS Lett 476(1-2): 89-92.

- Bacskai, B. J., J. Skoch, et al. (2003). "Fluorescence resonance energy transfer determinations using multiphoton fluorescence lifetime imaging microscopy to characterize amyloid-beta plaques." J Biomed Opt 8(3): 368-75. 
- Bagri, A. and M. Tessier-Lavigne (2002). "Neuropilins as Semaphorin receptors: in vivo functions in neuronal cell migration and axon guidance." Adv Exp Med Biol 515: 13-31.

- Barghorn, S. and E. Mandelkow (2002). "Toward a unified scheme for the aggregation of tau into Alzheimer paired helical filaments." Biochemistry 41(50): 14885-96.

- Barghorn, S., Q. Zheng-Fischhofer, et al. (2000). "Structure, microtubule interactions, and paired helical filament aggregation by tau mutants of frontotemporal dementias." Biochemistry 39(38): 11714-21.

- Bastiaens, P. I. and A. Squire (1999). "Fluorescence lifetime imaging microscopy: spatial resolution of biochemical processes in the cell." Trends Cell Biol 9(2): 48-52.

- Beaumont, V., N. Zhong, et al. (2001). "Phosphorylation and local presynaptic protein synthesis in calcium- and calcineurin-dependent induction of crayfish long-term facilitation." Neuron 32(3): 489-501.

- Beere, H. M. (2004). "'The stress of dying": the role of heat shock proteins in the regulation of apoptosis." J Cell Sci 117(Pt 13): 2641-51.

- Bence, N. F., R. M. Sampat, et al. (2001). "Impairment of the ubiquitinproteasome system by protein aggregation." Science 292(5521): 1552-5.

- Benech, J. C., M. Crispino, et al. (1999). "Protein synthesis in presynaptic endings from squid brain: modulation by calcium ions." J Neurosci Res 55(6): 776-81.

- Bennett, M. C., J. F. Bishop, et al. (1999). "Degradation of alpha-synuclein by proteasome." J Biol Chem 274(48): 33855-8.

- Bi, G. Q. and M. M. Poo (1998). "Synaptic modifications in cultured hippocampal neurons: dependence on spike timing, synaptic strength, and postsynaptic cell type.” J Neurosci 18(24): 10464-72.

- Bradke, F. and C. G. Dotti (1999). "The role of local actin instability in axon formation." Science 283(5409): 1931-4.

- Braisted, J. E., S. M. Catalano, et al. (2000). "Netrin-1 promotes thalamic axon growth and is required for proper development of the thalamocortical projection." J Neurosci 20(15): 5792-801.

- Branda, C. S. and M. J. Stern (1999). "Cell migration and axon growth cone guidance in Caenorhabditis elegans." Curr Opin Genet Dev 9(4): 479-84.

- Bray, D. and P. J. Hollenbeck (1988). "Growth cone motility and guidance." Annu Rev Cell Biol 4: 43-61. 
- Briknarova, K., S. Takayama, et al. (2001). "Structural analysis of BAG1 cochaperone and its interactions with Hsc70 heat shock protein." Nat Struct Biol 8(4): 349-52.

- Brittis, P. A., Q. Lu, et al. (2002). “Axonal protein synthesis provides a mechanism for localized regulation at an intermediate target." Cell 110(2): 223-35.

- Brose, K. and M. Tessier-Lavigne (2000). "Slit proteins: key regulators of axon guidance, axonal branching, and cell migration." Curr Opin Neurobiol 10(1): 95-102.

- Bulina, M. E., D. M. Chudakov, et al. (2002). "Interconversion of Anthozoa GFP-like fluorescent and non-fluorescent proteins by mutagenesis." BMC Biochem 3(1): 7.

- Calixto, E., E. Thiels, et al. (2003). "Early maintenance of hippocampal mossy fiber-long-term potentiation depends on protein and RNA synthesis and presynaptic granule cell integrity." J Neurosci 23(12): 4842-9.

- Calleja, V., S. M. Ameer-Beg, et al. (2003). "Monitoring conformational changes of proteins in cells by fluorescence lifetime imaging microscopy." Biochem J 372(Pt 1): 33-40.

- Casadio, A., K. C. Martin, et al. (1999). “A transient, neuron-wide form of CREB-mediated long-term facilitation can be stabilized at specific synapses by local protein synthesis." Cell 99(2): 221-37.

- Castellani, V., J. Falk, et al. (2004). "Semaphorin3A-induced receptor endocytosis during axon guidance responses is mediated by L1 CAM." Mol Cell Neurosci 26(1): 89-100.

- Challacombe, J. F., D. M. Snow, et al. (1996). “Actin filament bundles are required for microtubule reorientation during growth cone turning to avoid an inhibitory guidance cue.” J Cell Sci 109 ( Pt 8): 2031-40.

- Challacombe, J. F., D. M. Snow, et al. (1997). "Dynamic microtubule ends are required for growth cone turning to avoid an inhibitory guidance cue." Neurosci 17(9): 3085-95.

- Chan, F. K., R. M. Siegel, et al. (2001). "Fluorescence resonance energy transfer analysis of cell surface receptor interactions and signaling using spectral variants of the green fluorescent protein." Cytometry 44(4): 361-8.

- Chan, W. K., J. T. Yabe, et al. (2003). "Growth cones contain a dynamic population of neurofilament subunits." Cell Motil Cytoskeleton 54(3): 195207. 
- Chedotal, A., J. A. Del Rio, et al. (1998). "Semaphorins III and IV repel hippocampal axons via two distinct receptors." Development 125(21): 431323.

- Chen, H., A. Bagri, et al. (2000). "Neuropilin-2 regulates the development of selective cranial and sensory nerves and hippocampal mossy fiber projections." Neuron 25(1): 43-56.

- Chen, H., A. Chedotal, et al. (1997). "Neuropilin-2, a novel member of the neuropilin family, is a high affinity receptor for the semaphorins Sema E and Sema IV but not Sema III." Neuron 19(3): 547-59.

- Chen, H., Z. He, et al. (1998). "Semaphorin-neuropilin interactions underlying sympathetic axon responses to class III semaphorins." Neuron 21(6): 1283-90.

- Chen, H., Z. He, et al. (1998). "Axon guidance mechanisms: semaphorins as simultaneous repellents and anti-repellents.” Nat Neurosci 1(6): 436-9.

- Chen, Y., J. D. Mills, et al. (2003). "Protein localization in living cells and tissues using FRET and FLIM.” Differentiation 71(9-10): 528-41.

- Chen, Y. and A. Periasamy (2004). "Characterization of two-photon excitation fluorescence lifetime imaging microscopy for protein localization." Microsc Res Tech 63(1): 72-80.

- Cheng, H. J., A. Bagri, et al. (2001). "Plexin-A3 mediates semaphorin signaling and regulates the development of hippocampal axonal projections." Neuron 32(2): 249-63.

- Chuckowree, J. A. and J. C. Vickers (2003). "Cytoskeletal and morphological alterations underlying axonal sprouting after localized transection of cortical neuron axons in vitro." J Neurosci 23(9): 3715-25.

- Colamarino, S. A. and M. Tessier-Lavigne (1995). "The axonal chemoattractant netrin-1 is also a chemorepellent for trochlear motor axons." Cell 81(4): 621-9.

- Cooper, H. M. (2002). "Axon guidance receptors direct growth cone pathfinding: rivalry at the leading edge." Int J Dev Biol 46(4): 621-31.

- Cuervo, A. M., L. Stefanis, et al. (2004). "Impaired degradation of mutant alpha-synuclein by chaperone-mediated autophagy." Science 305(5688): 1292-5.

- Culotti, J. G. and D. C. Merz (1998). "DCC and netrins." Curr Opin Cell Biol 10(5): 609-13.

- Dabir, D. V., J. Q. Trojanowski, et al. (2004). "Expression of the small heatshock protein alphaB-crystallin in tauopathies with glial pathology." Am J Pathol 164(1): 155-66. 
- Dahl, D. and A. Bignami (1978). "Neurofilament protein in clonal lines of mouse neuroblastoma." Dev Neurosci 1(3-4): 142-52.

- Dantuma, N. P., K. Lindsten, et al. (2000). "Short-lived green fluorescent proteins for quantifying ubiquitin/proteasome-dependent proteolysis in living cells." Nat Biotechnol 18(5): 538-43.

- David, D. C., R. Layfield, et al. (2002). "Proteasomal degradation of tau protein." J Neurochem 83(1): 176-85.

- de la Torre, J. R., V. H. Hopker, et al. (1997). "Turning of retinal growth cones in a netrin-1 gradient mediated by the netrin receptor DCC." Neuron 19(6): 1211-24.

- Deiner, M. S., T. E. Kennedy, et al. (1997). "Netrin-1 and DCC mediate axon guidance locally at the optic disc: loss of function leads to optic nerve hypoplasia." Neuron 19(3): 575-89.

- Dent, E. W., A. M. Barnes, et al. (2004). "Netrin-1 and semaphorin 3A promote or inhibit cortical axon branching, respectively, by reorganization of the cytoskeleton." J Neurosci 24(12): 3002-12.

- Dent, E. W. and F. B. Gertler (2003). "Cytoskeletal dynamics and transport in growth cone motility and axon guidance.” Neuron 40(2): 209-27.

- Dent, E. W. and K. Kalil (2001). "Axon branching requires interactions between dynamic microtubules and actin filaments." J Neurosci 21(24): $9757-$ 69.

- Dent, E. W., F. Tang, et al. (2003). "Axon guidance by growth cones and branches: common cytoskeletal and signaling mechanisms." Neuroscientist 9(5): 343-53.

- Dickson, B. J. (2001). "Rho GTPases in growth cone guidance.” Curr Opin Neurobiol 11(1): 103-10.

- Dickson, B. J. and K. Keleman (2002). “Netrins.” Curr Biol 12(5): R154-5.

- Dickson, B. J. and K. A. Senti (2002). "Axon guidance: growth cones make an unexpected turn." Curr Biol 12(6): R218-20.

- Dillon, T. E., J. Saldanha, et al. (2004). "Sema3A regulates the timing of target contact by cranial sensory axons." J Comp Neurol 470(1): 13-24.

- Dou, F., W. J. Netzer, et al. (2003). "Chaperones increase association of tau protein with microtubules.” Proc Natl Acad Sci U S A 100(2): 721-6.

- Dou, F., W. J. Netzer, et al. (2003). "Chaperones increase association of tau protein with microtubules.” Proc Natl Acad Sci U S A 100(2): 721-6. 
- Drescher, U. (1996). "Netrins find their receptor.” Nature 384(6608): 416-7.

- Du, J. L. and M. M. Poo (2004). "Rapid BDNF-induced retrograde synaptic modification in a developing retinotectal system." Nature 429(6994): 878-83.

- Dwyer, D. S., Y. Liu, et al. (1996). "Neuronal differentiation in PC12 cells is accompanied by diminished inducibility of Hsp70 and Hsp60 in response to heat and ethanol." Neurochem Res 21(6): 659-66.

- Eguchi, Y., S. Shimizu, et al. (1997). "[Molecular biology of apoptosis]." Rinsho Byori 45(5): 470-6.

- Ehlers, M. D. (2004). "Deconstructing the axon: Wallerian degeneration and the ubiquitin-proteasome system." Trends Neurosci 27(1): 3-6.

- Eisen, J. S. (1988). "Growth cone guidance and pathway formation." Trends Neurosci 11(8): 333-5.

- Elsliger, M. A., R. M. Wachter, et al. (1999). "Structural and spectral response of green fluorescent protein variants to changes in pH." Biochemistry 38(17): 5296-301.

- Emptage, N. J. (2001). "Fluorescent imaging in living systems." Curr Opin Pharmacol 1(5): 521-5.

- Erickson, M. G., D. L. Moon, et al. (2003). "DsRed as a potential FRET partner with CFP and GFP." Biophys J 85(1): 599-611.

- Erskine, L., S. E. Williams, et al. (2000). "Retinal ganglion cell axon guidance in the mouse optic chiasm: expression and function of robos and slits." $\mathrm{J}$ Neurosci 20(13): 4975-82.

- Forcet, C., E. Stein, et al. (2002). "Netrin-1-mediated axon outgrowth requires deleted in colorectal cancer-dependent MAPK activation." Nature 417(6887): 443-7.

- Gadella, T. W., Jr., G. N. van der Krogt, et al. (1999). "GFP-based FRET microscopy in living plant cells.” Trends Plant Sci 4(7): 287-291.

- Gainer, H. and J. L. Barker (1974). "Synaptic regulation of specific protein synthesis in an identified neuron." Brain Res 78(2): 314-9.

- Gainer, H., H. C. Pant, et al. (1994). "Squid optic lobe synaptosomes: what can they tell us about presynaptic protein synthesis?" J Neurochem 63(1): 3879.

- Gallo, G. and P. C. Letourneau (2004). "Regulation of growth cone actin filaments by guidance cues." J Neurobiol 58(1): 92-102. 
- Gautier, I., M. Tramier, et al. (2001). "Homo-FRET microscopy in living cells to measure monomer-dimer transition of GFP-tagged proteins." Biophys J 80(6):3000-8.

- Giasson, B. I. and V. M. Lee (2003). "Are ubiquitination pathways central to Parkinson's disease?" Cell 114(1): 1-8.

- Gillespie, L. N. (2003). "Regulation of axonal growth and guidance by the neurotrophin family of neurotrophic factors." Clin Exp Pharmacol Physiol 30(10): 724-33.

- Giuditta, A., B. B. Kaplan, et al. (2002). "Axonal and presynaptic protein synthesis: new insights into the biology of the neuron." Trends Neurosci 25(8): 400-4.

- Glanzer, J. G. and J. H. Eberwine (2003). "Mechanisms of translational control in dendrites." Neurobiol Aging 24(8): 1105-11.

- Goldbaum, O., M. Oppermann, et al. (2003). "Proteasome inhibition stabilizes tau inclusions in oligodendroglial cells that occur after treatment with okadaic acid." J Neurosci 23(26): 8872-80.

- Goldbaum, O. and C. Richter-Landsberg (2004). "Proteolytic stress causes heat shock protein induction, tau ubiquitination, and the recruitment of ubiquitin to tau-positive aggregates in oligodendrocytes in culture." $\mathrm{J}$ Neurosci 24(25): 5748-57.

- Goodman, C. S. (1996). "Mechanisms and molecules that control growth cone guidance." Annu Rev Neurosci 19: 341-77.

- Gordon-Weeks, P. R. (2004). "Microtubules and growth cone function." $\underline{\mathrm{J}}$ Neurobiol 58(1): 70-83.

- Goshima, Y., H. Hori, et al. (1999). "Growth cone neuropilin-1 mediates collapsin-1/Sema III facilitation of antero- and retrograde axoplasmic transport." J Neurobiol 39(4): 579-89.

- Grune, T., T. Reinheckel, et al. (2002). "Ezrin turnover and cell shape changes catalyzed by proteasome in oxidatively stressed cells." Faseb J 16(12): 160210.

- Guirland, C., S. Suzuki, et al. (2004). "Lipid rafts mediate chemotropic guidance of nerve growth cones." Neuron 42(1): 51-62.

- Hailey, D. W., T. N. Davis, et al. (2002). "Fluorescence resonance energy transfer using color variants of green fluorescent protein." Methods Enzymol 351: 34-49.

- Harpur, A. G., F. S. Wouters, et al. (2001). "Imaging FRET between spectrally similar GFP molecules in single cells.” Nat Biotechnol 19(2): 167-9. 
- Haseloff, J., E. L. Dormand, et al. (1999). "Live imaging with green fluorescent protein." Methods Mol Biol 122: 241-59.

- Hatayama, T., H. Takahashi, et al. (1997). "Reduced induction of HSP70 in PC12 cells during neuronal differentiation.” J Biochem (Tokyo) 122(5): 90410.

- He, Y., W. Yu, et al. (2002). "Microtubule reconfiguration during axonal retraction induced by nitric oxide." J Neurosci 22(14): 5982-91.

- He, Z. and M. Tessier-Lavigne (1997). "Neuropilin is a receptor for the axonal chemorepellent Semaphorin III." Cell 90(4): 739-51.

- Henley, J. and M. M. Poo (2004). "Guiding neuronal growth cones using Ca2+ signals." Trends Cell Biol 14(6): 320-30.

- Hirao, M., N. Sato, et al. (1996). "Regulation mechanism of ERM (ezrin/radixin/moesin) protein/plasma membrane association: possible involvement of phosphatidylinositol turnover and Rho-dependent signaling pathway." J Cell Biol 135(1): 37-51.

- Huber, A. B., A. L. Kolodkin, et al. (2003). "Signaling at the growth cone: ligand-receptor complexes and the control of axon growth and guidance." Annu Rev Neurosci 26: 509-63.

- Jares-Erijman, E. A. and T. M. Jovin (2003). "FRET imaging.” Nat Biotechnol 21(11): 1387-95.

- Jensen, K. K., L. Martini, et al. (2001). "Enhanced fluorescence resonance energy transfer between spectral variants of green fluorescent protein through zinc-site engineering." Biochemistry 40(4): 938-45.

- Jiang, X., P. Coffino, et al. (2004). "Development of a method for screening short-lived proteins using green fluorescent protein." Genome Biol 5(10): R81.

- Job, C. and J. Eberwine (2001). "Identification of sites for exponential translation in living dendrites." Proc Natl Acad Sci U S A 98(23): 13037-42.

- Job, C. and J. Eberwine (2001). "Localization and translation of mRNA in dendrites and axons." Nat Rev Neurosci 2(12): 889-98.

- Johnson, G., L. M. Refolo, et al. (1993). "Heat-shocked neuronal PC12 cells reveal Alzheimer's disease-associated alterations in amyloid precursor protein and tau." Ann N Y Acad Sci 695: 194-7.

- Jones, J., R. Heim, et al. (2000). "Development and application of a GFPFRET intracellular caspase assay for drug screening." J Biomol Screen 5(5): 307-18. 
- Kalil, K. (1996). "Growth cone behaviors during axon guidance in the developing cerebral cortex.” Prog Brain Res 108: 31-40.

- Kaplan, B. B., Z. S. Lavina, et al. (2004). "Subcellular compartmentation of neuronal protein synthesis: new insights into the biology of the neuron." Ann N Y Acad Sci 1018: 244-54.

- Keino-Masu, K., M. Masu, et al. (1996). "Deleted in Colorectal Cancer (DCC) encodes a netrin receptor.” Cell 87(2): 175-85.

- Kermer, P., M. H. Digicaylioglu, et al. (2003). "BAG1 over-expression in brain protects against stroke." Brain Pathol 13(4): 495-506.

- Kermer, P., M. Krajewska, et al. (2002). "Bag1 is a regulator and marker of neuronal differentiation." Cell Death Differ 9(4): 405-13.

- Keynes, R. and G. M. Cook (1996). "Axons turn as netrins find their receptor." Neuron 17(6): 1031-4.

- Keynes, R. J. and G. M. Cook (1992). "Repellent cues in axon guidance.” Curr Opin Neurobiol 2(1): 55-9.

- Kim, D. H., A. R. Vaccaro, et al. (2003). "Molecular biology of cervical myelopathy and spinal cord injury: role of oligodendrocyte apoptosis." Spine J 3(6): 510-9.

- Klucken, J., Y. Shin, et al. (2004). "A single amino acid substitution differentiates Hsp70-dependent effects on alpha-synuclein degradation and toxicity." Biochem Biophys Res Commun 325(1): 367-73.

- Klucken, J., Y. Shin, et al. (2004). "Hsp70 Reduces alpha-Synuclein Aggregation and Toxicity." J Biol Chem 279(24): 25497-502.

- Kolodkin, A. L. (1996). "Semaphorins: mediators of repulsive growth cone guidance.” Trends Cell Biol 6(1): 15-22.

- Kolodkin, A. L. (1998). "Semaphorin-mediated neuronal growth cone guidance." Prog Brain Res 117: 115-32.

- Konopasek, I., K. Strzalka, et al. (2000). "Cold shock in Bacillus subtilis: different effects of benzyl alcohol and ethanol on the membrane organisation and cell adaptation." Biochim Biophys Acta 1464(1): 18-26.

- Korhonen, L. and D. Lindholm (2004). "The ubiquitin proteasome system in synaptic and axonal degeneration: a new twist to an old cycle." J Cell Biol 165(1): 27-30.

- Kudoh, M., D. A. Knee, et al. (2002). "Bag1 proteins regulate growth and survival of ZR-75-1 human breast cancer cells." Cancer Res 62(6): 1904-9. 
- Leonardo, E. D., L. Hinck, et al. (1997). "Guidance of developing axons by netrin-1 and its receptors." Cold Spring Harb Symp Quant Biol 62: 467-78.

- Lewis, A. K. and P. C. Bridgman (1992). "Nerve growth cone lamellipodia contain two populations of actin filaments that differ in organization and polarity." J Cell Biol 119(5): 1219-43.

- Li, W., K. K. Ma, et al. (1998). "Phosphorylation sensitizes microtubuleassociated protein tau to $\mathrm{Al}(3+)$-induced aggregation." Neurochem Res 23(12): 1467-76.

- Lin, W. and B. G. Szaro (1995). "Neurofilaments help maintain normal morphologies and support elongation of neurites in Xenopus laevis cultured embryonic spinal cord neurons." J Neurosci 15(12): 8331-44.

- Lindholm, T., M. K. Skold, et al. (2004). "Semaphorin and neuropilin expression in motoneurons after intraspinal motoneuron axotomy." Neuroreport 15(4): 649-54.

- Liu, B. P. and S. M. Strittmatter (2001). "Semaphorin-mediated axonal guidance via Rho-related G proteins." Curr Opin Cell Biol 13(5): 619-26.

- Liu, Y., E. Stein, et al. (2004). "Novel role for Netrins in regulating epithelial behavior during lung branching morphogenesis.” Curr Biol 14(10): 897-905.

- Livesey, F. J. (1999). "Netrins and netrin receptors." Cell Mol Life Sci 56(12): $62-8$.

- Magge, S. and J. R. Madsen (1997). "Molecular biology of axonal outgrowth. 2. Pathfinding and molecular guidance cues." Pediatr Neurosurg 27(4): 16875.

- McLean, P. J., J. Klucken, et al. (2004). “Geldanamycin induces Hsp70 and prevents alpha-synuclein aggregation and toxicity in vitro." Biochem Biophys Res Commun 321(3): 665-9.

- McNaught, K. S., C. W. Olanow, et al. (2001). "Failure of the ubiquitinproteasome system in Parkinson's disease." Nat Rev Neurosci 2(8): 589-94.

- Messersmith, E. K., E. D. Leonardo, et al. (1995). "Semaphorin III can function as a selective chemorepellent to pattern sensory projections in the spinal cord.” Neuron 14(5): 949-59.

- Ming, G. L., H. J. Song, et al. (1997). "cAMP-dependent growth cone guidance by netrin-1.” Neuron 19(6): 1225-35.

- Mueller, B. K. (1999). "Growth cone guidance: first steps towards a deeper understanding." Annu Rev Neurosci 22: 351-88. 
- Muller, B., B. Stahl, et al. (1990). "In vitro experiments on axonal guidance and growth-cone collapse.” J Exp Biol 153: 29-46.

- Neumann, S., F. Bradke, et al. (2002). "Regeneration of sensory axons within the injured spinal cord induced by intraganglionic cAMP elevation." Neuron 34(6): 885-93.

- Nishiyama, M., A. Hoshino, et al. (2003). "Cyclic AMP/GMP-dependent modulation of $\mathrm{Ca} 2+$ channels sets the polarity of nerve growth-cone turning." Nature 423(6943): 990-5.

- Nollen, E. A., J. F. Brunsting, et al. (2000). "Bag1 functions in vivo as a negative regulator of Hsp70 chaperone activity." Mol Cell Biol 20(3): 1083-8.

- Okabe, S. and N. Hirokawa (1989). "Rapid turnover of microtubule-associated protein MAP2 in the axon revealed by microinjection of biotinylated MAP2 into cultured neurons." Proc Natl Acad Sci U S A 86(11): 4127-31.

- Okada, A., R. Lansford, et al. (1999). "Imaging cells in the developing nervous system with retrovirus expressing modified green fluorescent protein." Exp Neurol 156(2): 394-406.

- Ormo, M., A. B. Cubitt, et al. (1996). "Crystal structure of the Aequorea victoria green fluorescent protein.” Science 273(5280): 1392-5.

- Palm, G. J. and A. Wlodawer (1999). "Spectral variants of green fluorescent protein.” Methods Enzymol 302: 378-94.

- Pascual, M., E. Pozas, et al. (2004). "Coordinated functions of Netrin-1 and Class 3 secreted Semaphorins in the guidance of reciprocal septohippocampal connections." Mol Cell Neurosci 26(1): 24-33.

- Periasamy, A. and R. N. Day (1999). "Visualizing protein interactions in living cells using digitized GFP imaging and FRET microscopy." Methods Cell Biol 58: 293-314.

- Petrucelli, L., D. Dickson, et al. (2004). "CHIP and Hsp70 regulate tau ubiquitination, degradation and aggregation." Hum Mol Genet 13(7): 703-14.

- Placzek, M., M. Tessier-Lavigne, et al. (1990). "Guidance of developing axons by diffusible chemoattractants." Cold Spring Harb Symp Quant Biol 55: 279-89.

- Plesset, J., C. Palm, et al. (1982). "Induction of heat shock proteins and thermotolerance by ethanol in Saccharomyces cerevisiae." Biochem Biophys Res Commun 108(3): 1340-5.

- Pollok, B. A. and R. Heim (1999). "Using GFP in FRET-based applications." Trends Cell Biol 9(2): 57-60. 
- Potter, S. M., C. M. Wang, et al. (1996). "Intravital imaging of green fluorescent protein using two-photon laser-scanning microscopy." Gene 173(1 Spec No): 25-31.

- Puschel, A. W. (1996). "The semaphorins: a family of axonal guidance molecules?" Eur J Neurosci 8(7): 1317-21.

- Qing, G., L. C. Ma, et al. (2004). "Cold-shock induced high-yield protein production in Escherichia coli." Nat Biotechnol 22(7): 877-82.

- Ramesh, V. (2004). "Merlin and the ERM proteins in Schwann cells, neurons and growth cones." Nat Rev Neurosci 5(6): 462-70.

- Richter-Landsberg, C. and N. G. Bauer (2004). "Tau-inclusion body formation in oligodendroglia: the role of stress proteins and proteasome inhibition." Int J Dev Neurosci 22(7): 443-51.

- Rizzu, P., M. Joosse, et al. (2000). "Mutation-dependent aggregation of tau protein and its selective depletion from the soluble fraction in brain of P301L FTDP-17 patients." Hum Mol Genet 9(20): 3075-82.

- Rodger, J., P. N. Vitale, et al. (2004). "EphA/ephrin-A interactions during optic nerve regeneration: restoration of topography and regulation of ephrinA2 expression." Mol Cell Neurosci 25(1): 56-68.

- Sacchetti, A., V. Cappetti, et al. (2001). "Green Fluorescent Protein variants fold differentially in prokaryotic and eukaryotic cells." J Cell Biochem 81(S36): 117-128.

- Sato, M., T. Ozawa, et al. (2002). "Fluorescent indicators for imaging protein phosphorylation in single living cells." Nat Biotechnol 20(3): 287-94.

- Schinder, A. F., B. Berninger, et al. (2000). "Postsynaptic target specificity of neurotrophin-induced presynaptic potentiation." Neuron 25(1): 151-63.

- Schwartzman, R. A. and J. A. Cidlowski (1993). "Apoptosis: the biochemistry and molecular biology of programmed cell death." Endocr Rev 14(2): 133-51.

- Sekar, R. B. and A. Periasamy (2003). "Fluorescence resonance energy transfer (FRET) microscopy imaging of live cell protein localizations." J Cell Biol 160(5): 629-33.

- Serafini, T., S. A. Colamarino, et al. (1996). "Netrin-1 is required for commissural axon guidance in the developing vertebrate nervous system." Cell 87(6): 1001-14.

- Serafini, T., T. E. Kennedy, et al. (1994). "The netrins define a family of axon outgrowth-promoting proteins homologous to C. elegans UNC-6." Cell 78(3): 409-24. 
- Siegel, R. M., F. K. Chan, et al. (2000). "Measurement of molecular interactions in living cells by fluorescence resonance energy transfer between variants of the green fluorescent protein." Sci STKE 2000(38): PL1.

- Song, H., G. Ming, et al. (1998). "Conversion of neuronal growth cone responses from repulsion to attraction by cyclic nucleotides." Science 281(5382): 1515-8.

- Song, H. and M. Poo (2001). "The cell biology of neuronal navigation." Nat Cell Biol 3(3): E81-8.

- Song, H. J. and M. M. Poo (1999). "Signal transduction underlying growth cone guidance by diffusible factors." Curr Opin Neurobiol 9(3): 355-63.

- Song, J., M. Takeda, et al. (2001). "Bag1-Hsp70 mediates a physiological stress signalling pathway that regulates Raf-1/ERK and cell growth." Nat Cell Biol 3(3): 276-82.

- Speese, S. D., N. Trotta, et al. (2003). "The ubiquitin proteasome system acutely regulates presynaptic protein turnover and synaptic efficacy." Curr Biol 13(11): 899-910.

- Sretavan, D. W. and L. F. Reichardt (1993). "Time-lapse video analysis of retinal ganglion cell axon pathfinding at the mammalian optic chiasm: growth cone guidance using intrinsic chiasm cues." Neuron 10(4): 761-77.

- Stefanis, L., K. E. Larsen, et al. (2001). "Expression of A53T mutant but not wild-type alpha-synuclein in PC12 cells induces alterations of the ubiquitindependent degradation system, loss of dopamine release, and autophagic cell death.” J Neurosci 21(24): 9549-60.

- Stein, E., Y. Zou, et al. (2001). "Binding of DCC by netrin-1 to mediate axon guidance independent of adenosine A2B receptor activation." Science 291(5510): 1976-82.

- Stoeckli, E. T. (1997). "Molecular mechanisms of growth cone guidance: stop and go?" Cell Tissue Res 290(2): 441-9.

- Strasser, G. A., N. A. Rahim, et al. (2004). "Arp2/3 is a negative regulator of growth cone translocation." Neuron 43(1): 81-94.

- Su, C. Y., K. Y. Chong, et al. (1998). "Constitutive and inducible hsp70s are involved in oxidative resistance evoked by heat shock or ethanol." J Mol Cell Cardiol 30(3): 587-98.

- Suhling, K., J. Siegel, et al. (2002). "Imaging the environment of green fluorescent protein.” Biophys J 83(6): 3589-95. 
- Sun, Q. L., J. Wang, et al. (2000). "Growth cone steering by receptor tyrosine phosphatase delta defines a distinct class of guidance cue." Mol Cell Neurosci 16(5): 686-95.

- Suo, Z., M. Wu, et al. (2003). "Rapid tau aggregation and delayed hippocampal neuronal death induced by persistent thrombin signaling." J Biol Chem 278(39): 37681-9.

- Squire, A. \& Bastiaens, P.I.H. Three dimensional image restoration in fluorescence lifetime imaging. J. Microsc. 193, 36-49 (1999).

- Schönle, A., Glatz, M. \& Hell, S.W. Four-dimensional multiphoton microscopy with time-correlated single-photon counting. Appl. Optics 39, 6306-6311 (2000).

- Sytsma, J., Vroom, J.M., Degrauw, C.J. \& Gerritsen, H.C. Time-gated fluorescence lifetime imaging and microvolume spectroscopy using twophoton excitation. J. Microsc. 191, 39-51 (1998).

- Takaoka, A., Adachi, M., Okuda, H., Sato, S., Yawata, A., Hinoda, Y., Takayama, S., Reed, J.C. and Imai, K. (1997) Anti-cell death activity promotes pulmonary metastasis of melanoma cells. Oncogene 14: 2971-2977

- Takayama, S., Sato, T., Krajewski, S., Kochel, K., Irie, S., Millan, J.A. and Reed, J.C. (1995) Cloning and functional analysis of BAG-1: a novel Bcl-2binding protein with anti-cell death activity. Cell 80: 279-284

- Takayama, S., Bimston, D.N., Matsuzawa, S., Freeman, B.C., Aime-Sempe, C., Xie, Z., Morimoto, R.I. and Reed, J.C. (1997) BAG-1 modulates the chaperone activity of Hsp70/Hsc70. Embo J 16: 4887-4896

- Takayama, S., Xie, Z. and Reed, J.C. (1999), An evolutionarily conserved family of Hsp70/Hsc70 molecular chaperone regulators. J Biol Chem, 274: 781-786

- Takayama, S. and Reed, J.C. (2001) Molecular chaperone targeting and regulation by BAG family proteins. Nat Cell Biol 3: E237-241

- Tamagnone, L., S. Artigiani, et al. (1999). "Plexins are a large family of receptors for transmembrane, secreted, and GPI-anchored semaphorins in vertebrates." Cell 99(1): 71-80.

- Terada, K. and Mori, M. (2000) Human DnaJ homologs dj2 and dj3, and bag1 are positive cochaperones of hsc70. J Biol Chem 275: 24728-24734

- Thomas, J.G. and Baneyx, F. (1996), Protein misfolding and inclusion body formation in recombinant Escherichia coli cells overexpressing heat-shock proteins. J Biol Chem 271: 11141-11147 
- Townsend, P.A., Cutress, R.I., Sharp, A., Brimmell, M. and Packham, G. (2003) BAG-1 prevents stress-induced long-term growth inhibition in breast cancer cells via a chaperone-dependent pathway. Cancer Res 63: 4150-4157

- Townsend, P.A., Cutress, R.I., Carroll, C.J., Lawrence, K.M., Scarabelli, T.M., Packham, G., Stephanou, A. and Latchman, D.S. (2004) BAG-1 proteins protect cardiac myocytes from simulated ischemia/reperfusioninduced apoptosis via an alternate mechanism of cell survival independent of the proteasome. J Biol Chem 279: 20723-20728

- Tessier-Lavigne, M. (1992). "Axon guidance by molecular gradients.” Curr Opin Neurobiol 2(1): 60-5.

- Tessier-Lavigne, M. (1994). “Axon guidance by diffusible repellants and attractants." Curr Opin Genet Dev 4(4): 596-601.

- Tessier-Lavigne, M. (1995). "Eph receptor tyrosine kinases, axon repulsion, and the development of topographic maps." Cell 82(3): 345-8.

- Tessier-Lavigne, M., M. Placzek, et al. (1988). "Chemotropic guidance of developing axons in the mammalian central nervous system." Nature 336(6201): 775-8.

- Tetzlaff, W. and M. A. Bisby (1989). "Neurofilament elongation into regenerating facial nerve axons.” Neuroscience 29(3): 659-66.

- Thomas, J. G. and F. Baneyx (1996). "Protein misfolding and inclusion body formation in recombinant Escherichia coli cells overexpressing Heat-shock proteins." J Biol Chem 271(19): 11141-7.

- Tofaris, G. K., R. Layfield, et al. (2001). “alpha-synuclein metabolism and aggregation is linked to ubiquitin-independent degradation by the proteasome." FEBS Lett 509(1): 22-6.

- Togari, A., M. Mogi, et al. (2000). "Expression of mRNA for axon guidance molecules, such as semaphorin-III, netrins and neurotrophins, in human osteoblasts and osteoclasts.” Brain Res 878(1-2): 204-9.

- Topell, S., J. Hennecke, et al. (1999). "Circularly permuted variants of the green fluorescent protein.” FEBS Lett 457(2): 283-9.

- Tramier, M., I. Gautier, et al. (2002). "Picosecond-hetero-FRET microscopy to probe protein-protein interactions in live cells.” Biophys J 83(6): 3570-7.

- Tran, P. T., A. Paoletti, et al. (2004). "Imaging green fluorescent protein fusions in living fission yeast cells." Methods 33(3): 220-5.

- Truong, K., A. Sawano, et al. (2001). "FRET-based in vivo Ca2+ imaging by a new calmodulin-GFP fusion molecule." Nat Struct Biol 8(12): 1069-73. 
- Tsai, H. H., M. Tessier-Lavigne, et al. (2003). "Netrin 1 mediates spinal cord oligodendrocyte precursor dispersal.” Development 130(10): 2095-105.

- Tsien, R. Y. (1998). “The green fluorescent protein.” Annu Rev Biochem 67: 509-44.

- van Horck, F. P., C. Weinl, et al. (2004). "Retinal axon guidance: novel mechanisms for steering." Curr Opin Neurobiol 14(1): 61-6.

- van Roessel, P. and A. H. Brand (2002). "Imaging into the future: visualizing gene expression and protein interactions with fluorescent proteins." Nat Cell Biol 4(1): E15-20.

- von Bergen, M., S. Barghorn, et al. (2001). "Mutations of tau protein in frontotemporal dementia promote aggregation of paired helical filaments by enhancing local beta-structure." J Biol Chem 276(51): 48165-74.

- Wallace, W., G. Johnson, et al. (1993). "Reversible phosphorylation of tau to form A68 in heat-shocked neuronal PC12 cells." Brain Res Mol Brain Res 19(1-2): 149-55.

- Walsh, K. H. and D. W. Crabb (1989). "The heat-shock response in cultured cells exposed to ethanol and its metabolites." J Lab Clin Med 114(5): 563-7.

- Walter, J., B. Muller, et al. (1990). “Axonal guidance by an avoidance mechanism.” J Physiol (Paris) 84(1): 104-10.

- Watanabe, A., W. K. Hong, et al. (2004). "Molecular aging of tau: disulfideindependent aggregation and non-enzymatic degradation in vitro and in vivo." J Neurochem 90(6): 1302-11.

- Watts, R. J., E. D. Hoopfer, et al. (2003). “Axon pruning during Drosophila metamorphosis: evidence for local degeneration and requirement of the ubiquitin-proteasome system." Neuron 38(6): 871-85.

- Winberg, M. L., J. N. Noordermeer, et al. (1998). "Plexin A is a neuronal semaphorin receptor that controls axon guidance." Cell 95(7): 903-16.

- Wouters, F. S. and P. I. Bastiaens (1999). "Fluorescence lifetime imaging of receptor tyrosine kinase activity in cells." Curr Biol 9(19): 1127-30.

- Xiang, Y., Y. Li, et al. (2002). "Nerve growth cone guidance mediated by G protein-coupled receptors." Nat Neurosci 5(9): 843-8.

- Yao, T. M., K. Tomoo, et al. (2003). "Aggregation analysis of the microtubule binding domain in tau protein by spectroscopic methods." J Biochem (Tokyo) 134(1): 91-9.

- Yoshikawa, S. and J. B. Thomas (2004). "Secreted cell signaling molecules in axon guidance." Curr Opin Neurobiol 14(1): 45-50. 
- Yuan, X. B., M. Jin, et al. (2003). "Signalling and crosstalk of Rho GTPases in mediating axon guidance." Nat Cell Biol 5(1): 38-45.

- Zal, T. and N. R. Gascoigne (2004). "Photobleaching-corrected FRET efficiency imaging of live cells." Biophys J 86(6): 3923-39.

- Zeiner, M. and Gehring, U. (1995) A protein that interacts with members of the nuclear hormone receptor family: identification and cDNA cloning. Proc Natl Acad Sci USA 92: 11465-11469

- Zeiner, M., Gebauer, M. and Gehring, U. (1997) Mammalian protein RAP46: an interaction partner and modulator of $70 \mathrm{kDa}$ heat shock proteins. Embo J 16: $5483-5490$

- Zhong, L.T., Sarafian, T., Kane, D.J., Charles, A.C., Mah, S.P., Edwards, R.H. and Bredesen, D.E. (1993) bcl-2 inhibits death of central neural cells induced by multiple agents. Proc Natl Acad Sci USA 90: 4533-4537

- Zhai, Q., J. Wang, et al. (2003). "Involvement of the ubiquitin-proteasome system in the early stages of wallerian degeneration." Neuron 39(2): 217-25.

- Zhang, B. (2004). "Design of FRET-based GFP probes for detection of protease inhibitors." Biochem Biophys Res Commun 323(2): 674-8.

- Zhang, J., R. E. Campbell, et al. (2002). "Creating new fluorescent probes for cell biology." Nat Rev Mol Cell Biol 3(12): 906-18.

- Zhang, X. and M. M. Poo (2002). "Localized synaptic potentiation by BDNF requires local protein synthesis in the developing axon." Neuron 36(4): 67588.

- Zheng, J. Q., T. K. Kelly, et al. (2001). “A functional role for intra-axonal protein synthesis during axonal regeneration from adult sensory neurons." $\mathrm{J}$ Neurosci 21(23): 9291-303.

- Zheng, J. Q., J. J. Wan, et al. (1996). "Essential role of filopodia in chemotropic turning of nerve growth cone induced by a glutamate gradient." $\underline{\mathrm{J}}$ Neurosci 16(3): 1140-9.

- Zhou, F. Q. and C. S. Cohan (2001). "Growth cone collapse through coincident loss of actin bundles and leading edge actin without actin depolymerization." J Cell Biol 153(5): 1071-84.

- Zhou, F. Q. and C. S. Cohan (2004). "How actin filaments and microtubules steer growth cones to their targets." J Neurobiol 58(1): 84-91.

- Zhou, F. Q., C. M. Waterman-Storer, et al. (2002). "Focal loss of actin bundles causes microtubule redistribution and growth cone turning." J Cell Biol 157(5): 839-49. 
- Zourlidou, A., M. D. Payne Smith, et al. (2004). "HSP27 but not HSP70 has a potent protective effect against alpha-synuclein-induced cell death in mammalian neuronal cells." J Neurochem 88(6): 1439-48.

- Förster, T. Zwischenmolekulare Energiewanderung und Fluoreszenz. Ann. Phys. (Leipz.) 2, 55-75 (1948).

- Wouters, F.S., Verveer, P.J. \& Bastiaens, P.I.H. Imaging biochemistry inside cells. Trends Cell. Biol. 11, 203-211 (2001).

- Chalfie, M., Tu, Y., Euskirchen, G., Ward, W.W. \& Prasher, D.C. Green fluorescent protein as a marker for gene expression. Science 263, 802-805 (1994).

- Clegg, R.M., Feddersen, B.A., Gratton, E. \& Jovin, T.M. Time resolved imaging fluorescence microscopy. Proc. SPIE 1640, 448-460 (1992).

- Tsien, R.Y. The green fluorescent protein. Annu. Rev. Biochem. 67, 509-544 (1998).

- Becker, W., Bergmann, A., Konig, K. \& Tirlapur, U. Picosecond fluorescence lifetime microscopy by TCSPC imaging. Proc. SPIE 4262, 414-419. (2001).

- Siegel, R.M. et al. Measurement of molecular interactions in living cells by fluorescence resonance energy transfer between variants of the green fluorescent protein. Sci. STKE 38,PL1

- Matz, M.V. et al. Fluorescent proteins from non-bioluminescent Anthozoa species. Nat. Biotechnol. 17, 969-973 (1999).

- Adams, S.R. et al. New biarsenic ligands and tetracystein motifs for protein labeling in vivo: synthesis and biological applications. J. Am. Chem. Soc. 124, 6063-6076 (2002).

- Campbell, R.E. et al. A monomeric red fluorescent protein. Proc. natl. Acad. Sci. USA 99, 7877-7882 (2002).

- Harpur, A.G. \& Bastiaens, P.I.H. Probing protein interactions using GFP and Fluorescence Resonance Energy Transfer, In Molecular Cloning, a laboratory manual. Cold Spring Harbor laboratory Press (third edition, eds. Sambrook, J. \& Russel, D.W.) Volume 3, 18.69-18.95 (2001).

- Kummer, A.D. et al. Dramatic reduction in fluorescence quantum yield in mutants of Green Fluorescent Protein due to fast internal conversion. Chem. Phys. 237, 183-193 (1998).

- Hänninen, P.E., Lehtelä, L. \& Hell, S.W. Two-and multiphoton excitation of conjugate-dyes using a continuous wave laser. Optics Comm. 130, 29-33 (1996). 
- Li, X. et al. Generation of destablized green fluorescent protein as a transcription reporter. J. Biol. Chem. 273, 34970-34975 (1998).

- Jovin, T.M. \& Arndt-Jovin, D.J. Luminescence digital imaging microscopy. Annu. Rev. Biophys. Chem. 18, 271-308 (1989).

- Harpur, A.G., Wouters, F.S. \& Bastiaens, P.I.H. Imaging FRET between spectrally similar GFP molecules in single cells. Nat Biotechnol. 19, 167-169 (2001).

- Bulina, M.E., Chudakov, D.M. Mudrik, N.N. \& Lukyanov, K.A. Interconversion of Anthozoa GFP-like fluorescent and non-fluorescent proteins by mutagenesis. BMC Biochem. 3:7 (2002).

- Fradkov, A.F. et al. Far-red fluorescent tag for protein labeling. Biochem. J. immediate publication, BJ20021191 (2002).

- Wiedenmann, J., Schenk, A., Rocker, C., Girod, A., Spindler, K.D. \& Nienhaus, G.U. A far-red fluorescent protein with fast maturation and reduced oligomerization tendency from Entacmaea quadricolor (Anthozoa, Actinaria). Proc. natl. Acad. Sci. USA 99, 11646-11651 (2002).

- Yanushevich, Y.G. et al. A strategy for the generation of non-aggregating mutants of Anthozoa fluorescent proteins. FEBS Lett. 511, 11-14 (2002).

- Alberti, S., Esser, C. and Hohfeld, J. (2003) BAG-1--a nucleotide exchange factor of Hsc70 with multiple cellular functions. Cell Stress Chaperones 8: $225-231$

- Bardelli, A., Longati, P., Albero, D., Goruppi, S., Schneider, C., Ponzetto, C. and Comoglio, P.M. (1996) HGF receptor associates with the anti-apoptotic protein BAG-1 and prevents cell death. Embo J 15: 6205-6212

- Beere, H.M., Wolf, B.B., Cain, K., Mosser, D.D., Mahboubi, A., Kuwana, T., Tailor, P., Morimoto, R.I., Cohen, G.M. and Green, D.R. (2000) Heat-shock protein 70 inhibits apoptosis by preventing recruitment of procaspase- 9 to the Apaf-1 apoptosome. Nat Cell Biol 2: 469-475

- Bukau, B. and Horwich, A.L. (1998) The Hsp70 and Hsp60 chaperone machines. Cell 92: 351-366

- Demand, J., Alberti, S., Patterson, C. and Hohfeld, J. (2001) Cooperation of a ubiquitin domain protein and an E3 ubiquitin ligase during chaperone/proteasome coupling. Curr Biol 11: 1569-1577

- Gassler, C.S., Wiederkehr, T., Brehmer, D., Bukau, B. and Mayer, M.P. (2001) Bag-1M accelerates nucleotide release for human Hsc70 and Hsp70 and can act concentration-dependent as positive and negative cofactor. J Biol Chem 276: 32538-32544 
- Gebauer, M., Zeiner, M. and Gehring, U. (1997) Proteins interacting with the molecular chaperone hsp70/hsc70: physical associations and effects on refolding activity. FEBS Lett 417: 109-113

- Hartl, F.U. and Hayer-Hartl, M. (2002) Molecular chaperones in the cytosol: from nascent chain to folded protein. Science 295: 1852-1858

- Hohfeld, J. and Jentsch, S. (1997) GrpE-like regulation of the hsc70 chaperone by the anti-apoptotic protein BAG-1. Embo J 16: 6209-6216

- Jaattela, M., Wissing, D., Kokholm, K., Kallunki, T. and Egeblad, M. (1998) Hsp70 exerts its anti-apoptotic function downstream of caspase-3-like proteases. Embo J 17: 6124-6134

- Jürgen, B., Lin, H.J., Riemschneider, S., Scharf, C., Neubauer, P., Schmid, R., Hecker, M. and Schweder, T. (2000) Monitoring of genes that respond to overproduction of an insoluble recomabinant protein in Escherichia coli glucose-limited fed-batch fermentations. Biotechnol Bioeng 70: 217-224

- Kermer, P., Krajewska, M., Zapata, J.M., Takayama, S., Mai, J., Krajewski, S. and Reed, J.C. (2002) Bag1 is a regulator and marker of neuronal differentiation. Cell Death Differ 9: 405-413

- Kermer, P., Digicaylioglu, M.H., Kaul, M., Zapata, J.M., Krajewska, M., Stenner-Liewen, F., Takayama, S., Krajewski, S., Lipton, S.A. and Reed, J.C. (2003) BAG1 overexpression in brain protects against stroke. Brain Pathol 13: 495-506

- Kudoh, M., Knee, D.A., Takayama, S. and Reed, J.C. (2002) Bag1 proteins regulate growth and survival of ZR-75-1 human breast cancer cells. Cancer Res 62: 1904-1909

- Luders, J., Demand, J., Papp, O. and Hohfeld, J. (2000) Distinct isoforms of the cofactor BAG-1 differentially affect Hsc70 chaperone function. J Biol Chem 275: 14817-14823

- Matsuzawa, S., Takayama, S., Froesch, B.A., Zapata, J.M. and Reed, J.C. (1998) p53-inducible human homologue of Drosophila seven in absentia (Siah) inhibits cell growth: suppression by BAG-1. Embo J 17: 2736-2747

- O'Neill, E. and Kolch, W. (2004) Conferring specificity on the ubiquitous Raf/MEK signalling pathway. Br J Cancer 90: 283-288

- Roth, W., Grimmel, C., Rieger, L., Strik, H., Takayama, S., Krajewski, S., Meyermann, R., Dichgans, J., Reed, J.C. and Weller, M. (2000) Bag-1 and Bcl-2 gene transfer in malignant glioma: modulation of cell cycle regulation and apoptosis. Brain Pathol 10: 223-234 
- Sacchetti, A., Cappetti, V., Marra, P., Dell'Arciprete, R., El Sewedy, T., Crescenzi, C. and Alberti, S. (2001) Green fluorescent protein variants fold differentially in prokaryotic and eukaryotic cells. J Cell Biochem 81: 117-128

- Saleh, A., Srinivasula, S.M., Balkir, L., Robbins, P.D. and Alnemri, E.S. (2000) Negative regulation of the Apaf-1 apoptosome by Hsp70. Nat Cell Biol 2: $476-483$

- Schulz, J.B., Bremen, D., Reed, J.C., Lommatzsch, J., Takayama, S., Wullner, U., Loschmann, P.A., Klockgether, T. and Weller, M. (1997) Cooperative interception of neuronal apoptosis by BCL-2 and BAG-1 expression: prevention of caspase activation and reduced production of reactive oxygen species. J Neurochem 69: 2075-2086

- Sippel, R.S. and Chen, H. (2002) Activation of the ras/raf-1 signal transduction pathway in carcinoid tumor cells results in morphologic transdifferentiation. Surgery 132: 1035-1039; discussion 1039

- Song, J., Takeda, M. and Morimoto, R.I. (2001) Bag1-Hsp70 mediates a physiological stress signalling pathway that regulates Raf-1/ERK and cell growth. Nat Cell Biol 3: 276-282

- Sondermann, H., Scheufler, C., Schneider, C., Hohfeld, J., Hartl, F.U. and Moarefi, I. (2001) Structure of a Bag/Hsc70 complex: convergent functional evolution of Hsp70 nucleotide exchange factors. Science 291: 1553-1557

- Wang, H.G., Takayama, S., Rapp, U.R. and Reed, J.C. (1996) Bcl-2 interacting protein, BAG-1, binds to and activates the kinase Raf- 1. Proc Natl Acad Sci USA 93: 7063-7068

- Young, J.C., Moarefi, I. and Hartl, F.U. (2001) Hsp90: a specialized but essential protein-folding tool. J Cell Biol 154: 267-273 


\section{Acknowledgement}

In this genuine opurtunity, I like to thank many people for their help, contribution and moral support during my stay of 3 years in germany

Many thanks to my doctor father Prof. Friedrich.W. Schürmann on deciding my ability to start the research carier in germany. I sincerely thank his interest in taking care of the quality of my research thesis and his critical comments throught out this work period. I extend my vote of thanks also to Prof. Detlef.Doenecke for aggreeing to be my co-referee of the thesis.

I must thank my supervisor Dr. Fred.S.Wouters, for his constant help and significant contribution in the learning process through out my ph.d thesis. Special thanks for his understanding and lenience during these years of my research carier which enabled me to seek a broader perspective in independent research. I also like to thank Marjan Rupnik and Stephan Sigrist for their notable influence during my stay in the ENI.

I extend my special thanks to Prof. Detlev Schild for his kindness and friendly attitude during my stay in graduate college GRK-723, it was such a great atmosphere he created among the students and his help of financial support on my thesis duration, has to be recorded invaluable.

There must be some special people in the life of every induvidual. Upon counting and thinking of thanking them, it striked me to owe my sincere thanks to people I admire Sarita, Christian, Phillipe, Simon, Alessandro, Miso, Ajay, Sadanand, Suzan, Irina, Eva and Fernanda for creating such a influence on my character and life. I absolutely have no words to thank them completely. Instead I like to express my deepest gratitude for being with me in all good and bad moments of life in germany.

I feel humbled to have people like Sundaraj, Kamesh and Rajasekar as true and genuine friends, It seems rather unlikely to live in far away place without their concerned emails and positive updates about my family back home. Thanks guys for keeping the moral high and taking care of my immediate needs in friendship.

I really have to express my sincere gratitude to Aspi, Manuela, for giving valuable suggestion to help this thesis get shape. My special thanks goes to Aspi for his contribution to my thesis work and helpful suggestion to finish the thesis in time. I also like to appreciate the help of Dirk lange for being with me all the time of my work and his excellent technical support at the first phase of my project.

My special thanks to people that I always adore, Weibke and Dagmar, I thank you both for providing me all the official details and let my stay in germany peaceful and happy. Without your help and concerns life would have been crazy for visitors like me. I like also to appreciate \& thank Magda, for her welcoming smile everyday.

All these great moments of happiness will not be complete, if I do not thank my family and friends back home, who always appreciate every moment of pride and success in my life during all these wonderful years. At this moment I like to extend a very warm and sincere thanks to my people, friends, and family in India. 
Lebenslauf

Name

Date of birth

Birth place

Nationality

Studies and Experience

1992-1994

1994-1997

1997-1999

1999-2000

2000-2001

2001-Present
Sundar Ganesan

09/11/1976

Kanchipuram

Indian

Higher secondary education, Anderson higher secondary school, kanchipuram, India.

Bachelor of Science in Biochemistry at Sri Sankara arts and Science College, Kanchipuram, India.

Master of Science in Biochemistry at The University of Madras, Madras, India.

Worked as a lecturer in Biochemistry at Sri Sankara Arts and Science College, Kanchipuram, India.

Worked as a project assistant with Prof. Ragavan Varadarajan at the Indican Institute of Science, Bangalore, India.

Joined the European Neuroscience InstituteGoettingen, Germany to pursue the Doctoral thesis under the supervision of Dr. Fred.S.Wouters on the project Local Protein turnover as the regulatory mechanism in the growth and collapse of the neuronal growth cones“" 